\title{
Enantioselective Assembly of Spironolactones through NHC-Catalyzed Remote $\gamma$-Carbon Addition of Enals with Isatins
}

Xianfeng Rong, ${ }^{a, b}$ Hong Yao, ${ }^{b}$ Wenjing Xia, ${ }^{b}$ Yonglei Du, ${ }^{a, b}$ Yu Zhou, ${ }^{b,{ }^{*}}$ Hong Liu $^{b, *}$

${ }^{a}$ Nano Science and Technology Institute, University of Science and Technology of China, 166 Ren Ai Road, Suzhou 215123, China.

${ }^{b}$ CAS Key Laboratory of Receptor Research, Shanghai Institute of Materia Medica, Chinese Academy of Sciences 555 Zuchongzhi Road, Shanghai 201203, P. R. China *E-mail: zhouyu@simm.ac.cn; hliu@mail.shcnc.ac.cn.

Part I General Information .2

Part II Experimental part ...................................................................................................................................

1.General procedure for synthesis of $\gamma$-aryloxyacetaldehydes $3 \mathrm{a}-3 \mathrm{v}$ 3

2. X-ray Structure of compound 3f. .17

3. References and notes 18

Part III NMR Spectra . .19

Part IV HPLC Spectra .42 


\section{Part I General Information}

All other chemicals were of commercial grade and used without further purification. tetrahydrofuran used in reactions was freshly dried from sodium. Toluene used in reactions was freshly distilled from sodium. Analytical thin-layer chromatography (TLC) was performed on HSGF 254 (0.15-0.2 $\mathrm{mm}$ thickness), visualized by irradiation with UV light (254 nm). Column Chromatography was performed with CombiFlash® companion system (Teledyne Isco. Cn), silica gel was purchased from

qingdaohaiyang (300-400 mesh). ${ }^{1} \mathrm{H}-\mathrm{NMR}$ and ${ }^{13} \mathrm{C}-\mathrm{NMR}$ were recorded on 400 or $500 \mathrm{MHz}$ spectrometer. Data are reported in the following order: chemical shift $(\delta)$ in ppm; multiplicities are indicated, s (singlet), d (doublet), t (triplet), q (quartet), m (multiplet); coupling constants (J) are in Hertz (Hz). HPLC was performed on a JASCO 2000 instrument by using Daicel columns. LC-MS was performed on an Agilent 1100 instrument by column Eclipse XDB-C18 $(4.6 \times 150 \mathrm{~mm}, 5 \mu \mathrm{m})$ or Extend-C18 $(4.6 \times 150 \mathrm{~mm}, 5 \mu \mathrm{m})$. 

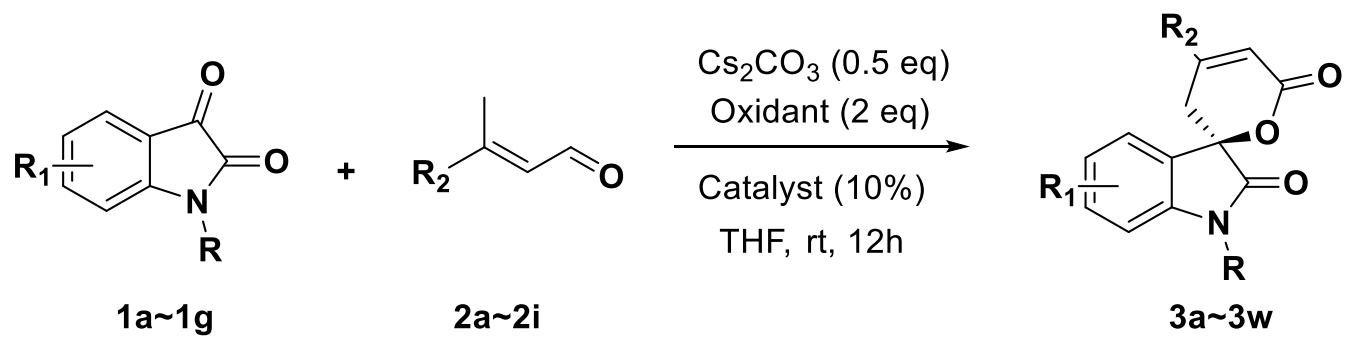

A screw-capped tube was charged with a solution of indole $\mathbf{1}^{\mathrm{a}}(0.25 \mathrm{mmol}, 1.0$ equiv) in THF $(2 \mathrm{~mL})$, then the solution was added $\alpha, \beta$-unsaturated aldehyde $\mathbf{2}^{\mathrm{b}}$ (0.50mmol, 2.0 equiv), NHC precursor $\mathbf{B}\left(0.025 \mathrm{mmol}, 0.1\right.$ equiv) and $\mathrm{Cs}_{2} \mathrm{CO}_{3}$ $(0.125 \mathrm{mmol}, 0.5$ equiv). After that the tube was placed under a positive pressure of $\mathrm{Ar}$ and the mixture was reacted at room temperature for $12 \mathrm{~h}$. After the completion of the reaction, the mixture was poured into water $(10 \mathrm{~mL})$, and extracted with EtOAc $(2 \mathrm{x}$ $10 \mathrm{~mL}$ ). The organic layer was dried over $\mathrm{Na}_{2} \mathrm{SO}_{4}$, filtered and concentrated in vacuo. The residue was purified by column chromatography on silica gel to afford the desired product.

(R)-1-methyl-4' -phenylspiro[indoline-3,2'-pyran]-2,6'(3'H)-dione (3a).

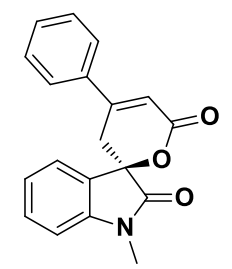

Yield 80\%; white solid; M.P.: $178.9-181.3{ }^{\circ} \mathrm{C} ; \mathrm{R}_{\mathrm{f}}=0.31$ (petroleum ether/ethyl acetate $=2 / 1) ;[\alpha]_{\mathrm{D}}^{25}=-25.6\left(\mathrm{c}=1.0, \mathrm{CHCl}_{3}\right) ;$ HPLC analysis: $81 \%$ ee [Daicel CHIRALPAK AD-H column, $20{ }^{\circ} \mathrm{C}, 254 \mathrm{~nm}$ hexane/i-PrOH $=85: 15,1.0 \mathrm{~mL} / \mathrm{min}$, $254 \mathrm{~nm}, 34.4 \mathrm{~min}$ (minor), $44.4 \mathrm{~min}$ (major)]; ${ }^{1} \mathrm{H} \mathrm{NMR}\left(\mathrm{CDCl}_{3}, 500 \mathrm{MHz}\right) \delta 7.56(\mathrm{dd}$, $J=7.8,1.8 \mathrm{~Hz}, 2 \mathrm{H}), 7.53-7.43(\mathrm{~m}, 4 \mathrm{H}), 7.41(\mathrm{t}, J=7.7 \mathrm{~Hz}, 1 \mathrm{H}), 7.08(\mathrm{t}, J=7.7 \mathrm{~Hz}$, 1H), $6.90(\mathrm{~d}, J=7.8 \mathrm{~Hz}, 1 \mathrm{H}), 6.61(\mathrm{~s}, 1 \mathrm{H}), 3.38(\mathrm{dd}, J=17.8,1.6 \mathrm{~Hz}, 1 \mathrm{H}), 3.25(\mathrm{~s}$,

$3 \mathrm{H}), 3.16-3.08(\mathrm{dd}, J=17.8,1.3 \mathrm{~Hz}, 1 \mathrm{H}) ;{ }^{13} \mathrm{C} \mathrm{NMR}\left(\mathrm{CDCl}_{3}, 125 \mathrm{MHz}\right) \delta 172.48$, 163.19 , 151.92, 143.17, 135.93, 131.10, 130.91, 129.08, 127.77, 126.05, 124.02, 123.46, 114.78, 108.96, 79.71, 32.51, 29.68, 26.49. LRMS (ESI) $[\mathrm{M}+\mathrm{H}]^{+}$found $\mathrm{m} / \mathrm{z}$ 
306, HRMS (ESI) calculated for $\mathrm{C}_{19} \mathrm{H}_{15} \mathrm{NO}_{3} \mathrm{Na}[\mathrm{M}+\mathrm{Na}]^{+}:$328.0950, found: 328.0948.

(R)-4' -(4-methoxyphenyl)-1-methylspiro[indoline-3,2'-pyran]-2,6' (3'H)-dione (3b).

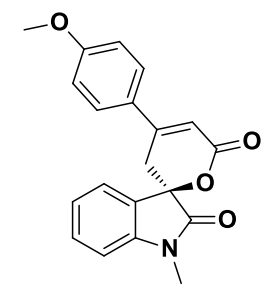

Yield 56\%; white solid; M.P.: $191.6-193.2{ }^{\circ} \mathrm{C} ; \mathrm{R}_{\mathrm{f}}=0.33$ (petroleum ether/ethyl acetate $=2 / 1) ;[\alpha]_{\mathrm{D}}^{25}=-32.5\left(\mathrm{c}=1.0, \mathrm{CHCl}_{3}\right) ; \mathrm{HPLC}$ analysis: $86 \%$ ee [Daicel CHIRALPAK OD-H column, $20{ }^{\circ} \mathrm{C}, 254 \mathrm{~nm}$ hexane/i-PrOH $=80: 20,1.0 \mathrm{~mL} / \mathrm{min}$, $254 \mathrm{~nm}, 48.1 \mathrm{~min}$ (major), $81.3 \mathrm{~min}$ (minor)]; ${ }^{1} \mathrm{H} \mathrm{NMR}\left(\mathrm{CDCl}_{3}, 500 \mathrm{MHz}\right): \delta 7.44$ (d, $J=8.9 \mathrm{~Hz}, 2 \mathrm{H}), 7.39(\mathrm{~d}, J=7.5 \mathrm{~Hz}, 1 \mathrm{H}), 7.30(\mathrm{td}, J=7.8,1.1 \mathrm{~Hz}, 1 \mathrm{H}), 6.96(\mathrm{t}, J=$ $7.9 \mathrm{~Hz}, 1 \mathrm{H}), 6.87(\mathrm{~d}, J=8.9 \mathrm{~Hz}, 2 \mathrm{H}), 6.80$ (d, $J=7.8 \mathrm{~Hz}, 1 \mathrm{H}), 6.45$ (s, 1H), 3.77 (s, $3 \mathrm{H}), 3.26(\mathrm{dd}, J=17.7,1.7 \mathrm{~Hz}, 1 \mathrm{H}), 3.15(\mathrm{~s}, 3 \mathrm{H}), 3.02-2.93(\mathrm{dd}, J=17.7,1.7 \mathrm{~Hz}$, $1 \mathrm{H}) ;{ }^{13} \mathrm{C} \mathrm{NMR}\left(\mathrm{CDCl}_{3}, 125 \mathrm{MHz}\right): \delta 172.56,163.52,161.95,151.34,143.18,131.04$, 127.99, 127.96, 127.74, 124.06, 123.44, 114.49, 112.42, 108.94, 79.65, 55.49, 32.27, 26.50, 1.03. LRMS (ESI) $[\mathrm{M}+\mathrm{H}]^{+}$found $\mathrm{m} / \mathrm{z}$ 336, HRMS (ESI) calculated for $\mathrm{C}_{20} \mathrm{H}_{17} \mathrm{NO}_{4} \mathrm{Na}[\mathrm{M}+\mathrm{Na}]^{+}:$358.1055, found: 358.1060 .

(R)-1-methyl-4' -(4-nitrophenyl)spiro[indoline-3,2'-pyran]-2,6' (3'H)-dione (3c).

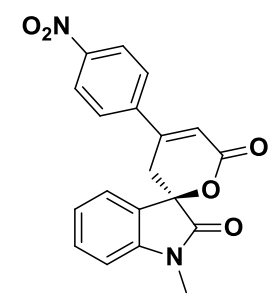

Yield 76\%; yellow solid; M.P.: $194.3-195.6{ }^{\circ} \mathrm{C} ; \mathrm{R}_{\mathrm{f}}=0.35$ (petroleum ether/ethyl acetate $=2 / 1) ;[\alpha]_{\mathrm{D}}^{25}=-21.4\left(\mathrm{c}=1.0, \mathrm{CHCl}_{3}\right) ;$ HPLC analysis: $79 \%$ ee [Daicel CHIRALPAK IA-H column, $20{ }^{\circ} \mathrm{C}, 254 \mathrm{~nm}$ hexane/i-PrOH = 85:15, $1.0 \mathrm{~mL} / \mathrm{min}, 254$ nm, 56.1 min (minor), $67.7 \mathrm{~min}$ (minor)]; ${ }^{1} \mathrm{H} \mathrm{NMR}\left(\mathrm{CDCl}_{3}, 500 \mathrm{MHz}\right): \delta 8.30(\mathrm{~d}, J=$ $8.8 \mathrm{~Hz}, 2 \mathrm{H}), 7.72(\mathrm{~d}, J=8.8 \mathrm{~Hz}, 2 \mathrm{H}), 7.48(\mathrm{~d}, J=7.6 \mathrm{~Hz}, 1 \mathrm{H}), 7.44(\mathrm{td}, J=7.8,1.2$ $\mathrm{Hz}, 1 \mathrm{H}), 7.13(\mathrm{td}, J=7.8,1.2 \mathrm{~Hz}, 1 \mathrm{H}), 6.92(\mathrm{~d}, J=7.6 \mathrm{~Hz}, 1 \mathrm{H}), 6.68(\mathrm{~s}, 1 \mathrm{H}), 3.26(\mathrm{dd}$, 
$J=17.8,1.3 \mathrm{~Hz}, 1 \mathrm{H}), 3.24(\mathrm{~m}, 4 \mathrm{H}) ;{ }^{13} \mathrm{C} \mathrm{NMR}\left(\mathrm{CDCl}_{3}, 125 \mathrm{MHz}\right): \delta 171.89,161.94$, $148.49,148.38,142.73,141.64,130.95,126.62,126.55,123.76,123.53,123.17$, 117.62, 108.65, 79.08, 32.17, 29.20, 26.02. LRMS (ESI) $[\mathrm{M}+\mathrm{H}]^{+}$found $\mathrm{m} / \mathrm{z} \mathrm{351}$, HRMS (ESI) calculated for $\mathrm{C}_{19} \mathrm{H}_{14} \mathrm{~N}_{2} \mathrm{O}_{5} \mathrm{Na}[\mathrm{M}+\mathrm{Na}]^{+}: 373.0800$, found: 373.0798 .

(R)-4' -(4-fluorophenyl)-1-methylspiro[indoline-3,2'-pyran]-2,6'(3'H)-dione (3d).

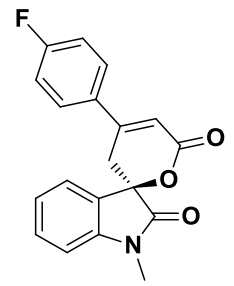

Yield 78\%; yellow solid; M.P.: 205.8-207.6 ${ }^{\circ} \mathrm{C} ; \mathrm{R}_{\mathrm{f}}=0.34$ (petroleum ether/ethyl acetate $=2 / 1) ;[\alpha]_{\mathrm{D}}^{25}=-22.5\left(\mathrm{c}=1.0, \mathrm{CHCl}_{3}\right) ;$ HPLC analysis: $75 \%$ ee [Daicel CHIRALPAK OD-H column, $20{ }^{\circ} \mathrm{C}, 254 \mathrm{~nm}$ hexane/i-PrOH $=80: 20,1.0 \mathrm{~mL} / \mathrm{min}$, $254 \mathrm{~nm}, 32.8 \mathrm{~min}$ (major), $47.9 \mathrm{~min}$ (minor)]; ${ }^{1} \mathrm{H} \mathrm{NMR}\left(\mathrm{CDCl}_{3}, 500 \mathrm{MHz}\right): \delta$ 7.49-7.41 (m, 2H), $7.36(\mathrm{~d}, J=7.5 \mathrm{~Hz}, 1 \mathrm{H}), 7.30(\mathrm{td}, J=7.8,1.2 \mathrm{~Hz}, 1 \mathrm{H}), 7.03(\mathrm{t}, J=$ $8.6 \mathrm{~Hz}, 2 \mathrm{H}), 6.97(\mathrm{t}, J=7.6 \mathrm{~Hz}, 1 \mathrm{H}), 6.79(\mathrm{~d}, J=7.8 \mathrm{~Hz}, 1 \mathrm{H}), 6.44(\mathrm{~s}, 1 \mathrm{H}), 3.20(\mathrm{dd}$, $J=17.8,1.3 \mathrm{~Hz}, 1 \mathrm{H}), 3.12(\mathrm{~s}, 3 \mathrm{H}), 2.99(\mathrm{dd}, J=17.8,1.3 \mathrm{~Hz}, 1 \mathrm{H}) ;{ }^{13} \mathrm{C} \mathrm{NMR}\left(\mathrm{CDCl}_{3}\right.$, $125 \mathrm{MHz}): \delta 172.48,165.28,163.27,163.13,150.62,143.19,132.06,132.04,131.21$, $128.19,128.12$, 127.64, 124.03, 123.54, 116.38, 116.21, 114.61, 109.04, 79.63, 32.57, 26.53. LRMS (ESI) $[\mathrm{M}+\mathrm{H}]^{+}$found $\mathrm{m} / \mathrm{z}$ 324, HRMS (ESI) calculated for $\mathrm{C}_{19} \mathrm{H}_{14} \mathrm{NO}_{3} \mathrm{NaF}[\mathrm{M}+\mathrm{Na}]^{+}: 346.0855$, found: 346.0856 .

(R)-4' -(4-chlorophenyl)-1-methylspiro[indoline-3,2'-pyran]-2,6' (3'H)-dione (3e).

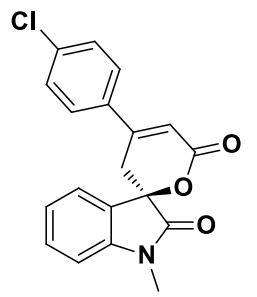

Yield 77\%; white solid; M.P.: $216.8-218.6{ }^{\circ} \mathrm{C} ; \mathrm{R}_{\mathrm{f}}=0.36$ (petroleum ether/ethyl acetate $=2 / 1) ;[\alpha]_{\mathrm{D}}{ }^{25}=-30.6\left(\mathrm{c}=1.0, \mathrm{CHCl}_{3}\right) ; \mathrm{HPLC}$ analysis: $82 \%$ ee [Daicel CHIRALPAK OD-H column, $20{ }^{\circ} \mathrm{C}, 254 \mathrm{~nm}$ hexane/i-PrOH $=80: 20,1.0 \mathrm{~mL} / \mathrm{min}$, 
$254 \mathrm{~nm}, 40.6 \mathrm{~min}$ (major), $73.5 \mathrm{~min}$ (minor)]; ${ }^{1} \mathrm{H} \mathrm{NMR}\left(\mathrm{CDCl}_{3}, 500 \mathrm{MHz}\right): \delta 7.50$ (d, $J=8.7 \mathrm{~Hz}, 2 \mathrm{H}), 7.48-7.45(\mathrm{~m}, 1 \mathrm{H}), 7.45-7.40(\mathrm{~m}, 3 \mathrm{H}), 7.09$ (t, $J=7.6 \mathrm{~Hz}, 1 \mathrm{H}), 6.90$ (d, $J=7.8 \mathrm{~Hz}, 1 \mathrm{H}), 6.59$ (s, 1H), 3.30 (dd, $J=17.8,1.5 \mathrm{~Hz}, 1 \mathrm{H}), 3.24$ (s, 3H), 3.11 $(\mathrm{dd}, J=17.8,1.5 \mathrm{~Hz}, 1 \mathrm{H}) ;{ }^{13} \mathrm{C} \mathrm{NMR}\left(\mathrm{CDCl}_{3}, 125 \mathrm{MHz}\right): \delta 172.43,171.14,162.97$, $150.40,143.18$, 137.09, 134.32, 131.20, 129.36, 127.32, 123.99, 123.52, 115.15, 109.00, 79.59, 32.45, 26.49, 14.18. LRMS (ESI) $[\mathrm{M}+\mathrm{H}]^{+}$found m/z 340, HRMS (ESI) calculated for $\mathrm{C}_{19} \mathrm{H}_{14} \mathrm{NO}_{3} \mathrm{NaCl}[\mathrm{M}+\mathrm{Na}]^{+}: 362.0560$, found: 362.0556 .

(R)-4' -(4-bromophenyl)-1-methylspiro[indoline-3,2'-pyran]-2,6' (3' H)-dione (3f).

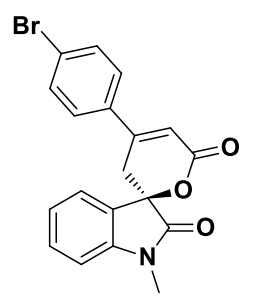

Yield 82\%; white solid; M.P.: $227.7-229.5{ }^{\circ} \mathrm{C} ; \mathrm{R}_{\mathrm{f}}=0.33$ (petroleum ether/ethyl acetate $=2 / 1) ;[\alpha]_{\mathrm{D}}{ }^{25}=-37.4\left(\mathrm{c}=1.0, \mathrm{CHCl}_{3}\right) ; \mathrm{HPLC}$ analysis: $81 \%$ ee [Daicel CHIRALPAK IA-H column, $20{ }^{\circ} \mathrm{C}, 254 \mathrm{~nm}$ hexane/i-PrOH = 80:20, $1.0 \mathrm{~mL} / \mathrm{min}, 254$ nm, $41.6 \mathrm{~min}$ (minor), $60.5 \mathrm{~min}$ (major)]; ${ }^{1} \mathrm{H} \mathrm{NMR}\left(\mathrm{CDCl}_{3}, 500 \mathrm{MHz}\right): \delta 7.59$ (d, $J=$ $8.6 \mathrm{~Hz}, 2 \mathrm{H}), 7.44(\mathrm{~d}, J=8.6 \mathrm{~Hz}, 2 \mathrm{H}), 7.47(\mathrm{~d}, J=7.6 \mathrm{~Hz}, 1 \mathrm{H}), 7.41(\mathrm{td}, J=7.3,1.3$ Hz, 1H), 7.09 (td, $J=7.3,1.3 \mathrm{~Hz}, 1 \mathrm{H}), 6.91$ (d, $J=7.6 \mathrm{~Hz}, 1 \mathrm{H}), 6.59$ (s, 1H), 3.30 (dd, $J=17.8,1.3 \mathrm{~Hz}, 1 \mathrm{H}), 3.24(\mathrm{~s}, 3 \mathrm{H}), 3.12(\mathrm{dd}, J=17.8,1.3 \mathrm{~Hz}, 1 \mathrm{H}) ;{ }^{13} \mathrm{C} \mathrm{NMR}\left(\mathrm{CDCl}_{3}\right.$, $125 \mathrm{MHz}): \delta 172.42,162.99,150.52,143.17,134.77,132.33,131.23,127.53,125.44$, 124.00, 123.54, 115.16, 109.04, 79.61, 32.39, 26.51. LRMS (ESI) $[\mathrm{M}+\mathrm{H}]^{+}$found $\mathrm{m} / \mathrm{z}$ 384, HRMS (ESI) calculated for $\mathrm{C}_{19} \mathrm{H}_{14} \mathrm{NO}_{3} \mathrm{NaBr}[\mathrm{M}+\mathrm{Na}]^{+}$: 406.0055, found: $406.0045,408.0013$

(R)-4' -(furan-2-yl)-1-methylspiro[indoline-3,2'-pyran]-2,6' (3'H)-dione (3g).

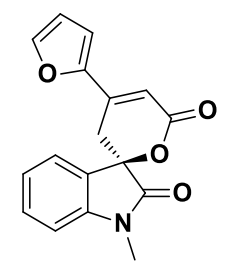

Yield 71\%; yellow solid; M.P.: $132.7-134.8^{\circ} \mathrm{C} ; \mathrm{R}_{\mathrm{f}}=0.35$ (petroleum ether/ethyl 
acetate $=2 / 1) ;[\alpha]_{\mathrm{D}}^{25}=-32.7\left(\mathrm{c}=1.0, \mathrm{CHCl}_{3}\right) ; \mathrm{HPLC}$ analysis: $82 \%$ ee [Daicel CHIRALPAK AD-H column, $20{ }^{\circ} \mathrm{C}, 254 \mathrm{~nm}$ hexane/i-PrOH $=85: 15,1.0 \mathrm{~mL} / \mathrm{min}$, $254 \mathrm{~nm}, 34.9 \mathrm{~min}$ (minor), $54.0 \mathrm{~min}$ (major)]; ${ }^{1} \mathrm{H} \mathrm{NMR}\left(\mathrm{CDCl}_{3}, 500 \mathrm{MHz}\right): \delta 7.60$ (d, $J=1.7 \mathrm{~Hz}, 1 \mathrm{H}), 7.46(\mathrm{~d}, J=7.6 \mathrm{~Hz}, 1 \mathrm{H}), 7.40(\mathrm{td}, J=7.7,1.2 \mathrm{~Hz}, 1 \mathrm{H}), 7.06(\mathrm{td}, J=$ 7.7, 1.2Hz, 1H), 6.89 (d, $J=7.6 \mathrm{~Hz}, 1 \mathrm{H}), 6.71(\mathrm{~d}, J=3.5 \mathrm{~Hz}, 1 \mathrm{H}), 6.58(\mathrm{~s}, 1 \mathrm{H}), 6.53$ $(\mathrm{dd}, J=3.5,1.7 \mathrm{~Hz}, 1 \mathrm{H}), 3.27(\mathrm{dd}, J=17.6,1.2 \mathrm{~Hz}, 1 \mathrm{H}), 3.23(\mathrm{~s}, 3 \mathrm{H}), 2.98(\mathrm{dd}, J=$ 17.6, $1.2 \mathrm{~Hz}, 1 \mathrm{H}) ;{ }^{13} \mathrm{C} \mathrm{NMR}\left(\mathrm{CDCl}_{3}, 125 \mathrm{MHz}\right): \delta 171.92,162.89,149.63,145.52$, $142.74,139.36,130.70,127.17,123.71,123.06,113.33,112.17,109.88,108.52$, 79.05, 29.76, 26.06. LRMS (ESI) $[\mathrm{M}+\mathrm{H}]^{+}$found m/z 296, HRMS (ESI) calculated for $\mathrm{C}_{17} \mathrm{H}_{13} \mathrm{NO}_{4} \mathrm{Na}[\mathrm{M}+\mathrm{Na}]^{+}: 318.0742$, found: 318.0739 .

(R)-4'-cyclohexyl-1-methylspiro[indoline-3,2'-pyran]-2,6'(3'H)-dione (3h).

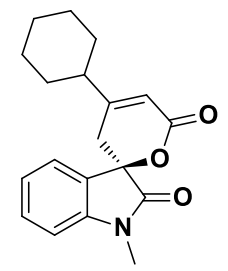

Yield 76\%; yellow solid; M.P.: $139.7-142.8{ }^{\circ} \mathrm{C} ; \mathrm{R}_{\mathrm{f}}=0.37$ (petroleum ether/ethyl acetate $=2 / 1) ;[\alpha]_{\mathrm{D}}{ }^{25}=-2.7\left(\mathrm{c}=1.0, \mathrm{CHCl}_{3}\right) ;$ HPLC analysis: $66 \%$ ee [Daicel CHIRALPAK OD-H column, $20{ }^{\circ} \mathrm{C}, 254 \mathrm{~nm}$ hexane/i-PrOH $=80: 20,1.0 \mathrm{~mL} / \mathrm{min}$, $254 \mathrm{~nm}, 15.7 \mathrm{~min}$ (major), $19.9 \mathrm{~min}$ (minor)]; ${ }^{1} \mathrm{H}$ NMR $\left(\mathrm{CDCl}_{3}, 500 \mathrm{MHz}\right): \delta 7.40(\mathrm{~d}$, $J=7.6 \mathrm{~Hz}, 1 \mathrm{H}), 7.37(\mathrm{td}, J=7.8,1.2 \mathrm{~Hz}, 1 \mathrm{H}), 7.05(\mathrm{td}, J=7.8,1.2 \mathrm{~Hz}, 1 \mathrm{H}), 6.86(\mathrm{~d}$, $J=7.6 \mathrm{~Hz}, 1 \mathrm{H}), 6.02(\mathrm{~s}, 1 \mathrm{H}), 3.20(\mathrm{~s}, 3 \mathrm{H}), 2.94(\mathrm{dd}, J=17.6,1.2 \mathrm{~Hz}, 1 \mathrm{H}), 2.60(\mathrm{dd}, J$ $=17.6,1.2 \mathrm{~Hz}, 1 \mathrm{H}), 1.88-1.79(\mathrm{~m}, 4 \mathrm{H}), 1.72(\mathrm{~m}, 1 \mathrm{H}), 1.28-1.15(\mathrm{~m}, 6 \mathrm{H}) ;{ }^{13} \mathrm{C} \mathrm{NMR}$ $\left(\mathrm{CDCl}_{3}, 125 \mathrm{MHz}\right): \delta 172.10,162.79,161.97,142.65,130.53,127.53,123.42,122.86$, 113.52, 108.49, 79.33, 44.40, 32.17, 29.80, 29.71, 26.02, 25.46, 25.40. LRMS (ESI) $[\mathrm{M}+\mathrm{H}]^{+}$found $\mathrm{m} / \mathrm{z}$ 312, HRMS (ESI) calculated for $\mathrm{C}_{19} \mathrm{H}_{21} \mathrm{NO}_{3} \mathrm{Na}[\mathrm{M}+\mathrm{Na}]^{+}$: 334.1419, found: 334.1409 .

(R)-1,4'-dimethylspiro[indoline-3,2'-pyran]-2,6'(3'H)-dione (3i). 


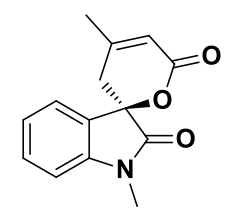

Yield 72\%; white solid; M.P.: $170.7-172.8{ }^{\circ} \mathrm{C} ; \mathrm{R}_{\mathrm{f}}=0.36$ (petroleum ether/ethyl acetate $=2 / 1) ;[\alpha]_{\mathrm{D}}^{25}=-22.0\left(\mathrm{c}=1.0, \mathrm{CHCl}_{3}\right) ; \mathrm{HPLC}$ analysis: $70 \%$ ee [Daicel CHIRALPAK OD-H column, $20{ }^{\circ} \mathrm{C}, 254 \mathrm{~nm}$ hexane/i-PrOH $=80: 20,1.0 \mathrm{~mL} / \mathrm{min}$, $254 \mathrm{~nm}, 24.7 \mathrm{~min}$ (major), $48.3 \mathrm{~min}$ (minor)]; ${ }^{1} \mathrm{H} \mathrm{NMR}\left(\mathrm{CDCl}_{3}, 500 \mathrm{MHz}\right): \delta 7.43$ (d, $J=7.6 \mathrm{~Hz}, 1 \mathrm{H}), 7.40(\mathrm{td}, J=7.7,1.2 \mathrm{~Hz}, 1 \mathrm{H}), 7.09(\mathrm{td}, J=7.7,1.2 \mathrm{~Hz}, 1 \mathrm{H}), 6.88(\mathrm{~d}$, $J=7.6 \mathrm{~Hz}, 1 \mathrm{H}), 6.08(\mathrm{~h}, J=1.6 \mathrm{~Hz}, 1 \mathrm{H}), 3.22(\mathrm{~s}, 3 \mathrm{H}), 2.88(\mathrm{~d}, J=18.2 \mathrm{~Hz}, 1 \mathrm{H}), 2.63$ $(\mathrm{d}, J=18.2 \mathrm{~Hz}, 1 \mathrm{H}), 2.07(\mathrm{~s}, 3 \mathrm{H}) ;{ }^{13} \mathrm{C} \mathrm{NMR}\left(\mathrm{CDCl}_{3}, 125 \mathrm{MHz}\right): \delta 172.63,162.74$, 154.29, 143.14, 131.03, 127.90, 123.88, 123.44, 116.53, 108.92, 79.63, 35.13, 26.45, 23.27. LRMS (ESI) $[\mathrm{M}+\mathrm{H}]^{+}$found $\mathrm{m} / \mathrm{z}$ 244, HRMS (ESI) calculated for $\mathrm{C}_{14} \mathrm{H}_{13} \mathrm{NO}_{3} \mathrm{Na}[\mathrm{M}+\mathrm{Na}]^{+}:$266.0793, found: 266.0786 .

(R)-6-chloro-1-methyl-4' -phenylspiro[indoline-3,2'-pyran]-2,6'(3'H)-dione (3j).

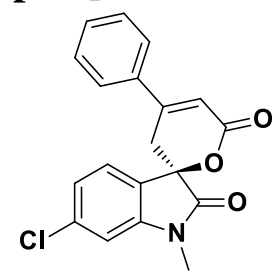

Yield 58\%; white solid; M.P.: $168.3-170.6{ }^{\circ} \mathrm{C} ; \mathrm{R}_{\mathrm{f}}=0.38$ (petroleum ether/ethyl acetate $=2 / 1) ;[\alpha]_{\mathrm{D}}{ }^{25}=-43.8\left(\mathrm{c}=1.0, \mathrm{CHCl}_{3}\right)$; HPLC analysis: $36 \%$ ee [Daicel CHIRALPAK OD-H column, $20{ }^{\circ} \mathrm{C}, 254 \mathrm{~nm}$ hexane/i-PrOH $=80: 20,1.0 \mathrm{~mL} / \mathrm{min}$, $254 \mathrm{~nm}, 38.0 \mathrm{~min}$ (major), $82.5 \mathrm{~min}$ (minor)]; ${ }^{1} \mathrm{H} \mathrm{NMR}\left(\mathrm{CDCl}_{3}, 500 \mathrm{MHz}\right): \delta 7.54$ (dd, $J=7.8,1.6 \mathrm{~Hz}, 2 \mathrm{H}), 7.51-7.43(\mathrm{~m}, 3 \mathrm{H}), 7.40(\mathrm{~d}, J=8.0 \mathrm{~Hz}, 1 \mathrm{H}), 7.04(\mathrm{dd}, J=8.0$, $1.8 \mathrm{~Hz}, 1 \mathrm{H}), 6.90$ (d, $J=1.8 \mathrm{~Hz}, 1 \mathrm{H}), 6.59$ (s, 1H), 3.33 (dd, $J=17.8,1.1 \mathrm{~Hz}, 1 \mathrm{H})$, $3.22(\mathrm{~s}, 3 \mathrm{H}), 3.10(\mathrm{dd}, J=17.8,1.1 \mathrm{~Hz}, 1 \mathrm{H}) ;{ }^{13} \mathrm{C} \mathrm{NMR}\left(\mathrm{CDCl}_{3}, 125 \mathrm{MHz}\right): \delta 171.98$, $162.45,151.38,144.03,136.66,135.31,130.64,128.72,125.63,124.61,122.87$, 114.28, 109.38, 78.81, 32.01, 29.27, 26.21. LRMS (ESI) $[\mathrm{M}+\mathrm{H}]^{+}$found $\mathrm{m} / \mathrm{z} 340$, HRMS (ESI) calculated for $\mathrm{C}_{19} \mathrm{H}_{14} \mathrm{NO}_{3} \mathrm{NaCl}[\mathrm{M}+\mathrm{Na}]^{+}$: 362.0560 , found: 361.0556 .

(R)-6-chloro-4' -(4-methoxyphenyl)-1-methylspiro[indoline-3,2'-pyran]-2,6' (3'H)- 
dione (3k).

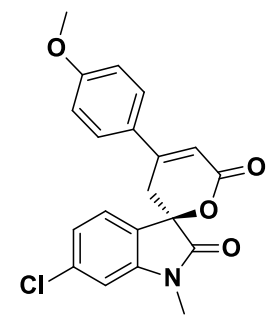

Yield 61\%; yellow solid; M.P.: 209.6-211.5 ${ }^{\circ} \mathrm{C} ; \mathrm{R}_{\mathrm{f}}=0.31$ (petroleum ether/ethyl acetate $=2 / 1) ;[\alpha]_{\mathrm{D}}^{25}=-58.7\left(\mathrm{c}=1.0, \mathrm{CHCl}_{3}\right) ; \mathrm{HPLC}$ analysis: $97 \%$ ee [Daicel CHIRALPAK AD-H column, $20{ }^{\circ} \mathrm{C}, 254 \mathrm{~nm}$ hexane/i-PrOH $=85: 15,1.0 \mathrm{~mL} / \mathrm{min}$, $254 \mathrm{~nm}, 63.9 \mathrm{~min}$ (minor), $76.1 \mathrm{~min}$ (major)]; ${ }^{1} \mathrm{H} \mathrm{NMR}\left(\mathrm{CDCl}_{3}, 500 \mathrm{MHz}\right): \delta 7.51$ (d, $J=8.8 \mathrm{~Hz}, 2 \mathrm{H}), 7.39(\mathrm{~d}, J=8.0 \mathrm{~Hz}, 1 \mathrm{H}), 7.02(\mathrm{dd}, J=8.0,1.5 \mathrm{~Hz}, 1 \mathrm{H}), 6.95(\mathrm{~d}, J=$ $8.8 \mathrm{~Hz}, 2 \mathrm{H}), 6.89$ (d, $J=1.5 \mathrm{~Hz}, 1 \mathrm{H}), 6.52(\mathrm{~s}, 1 \mathrm{H}), 3.86$ (s, 3H), 3.35 (dd, $J=17.8$, $1.1 \mathrm{~Hz}, 1 \mathrm{H}), 3.22(\mathrm{~s}, 3 \mathrm{H}), 3.04(\mathrm{dd}, J=17.8,1.1 \mathrm{~Hz}, 1 \mathrm{H}) ;{ }^{13} \mathrm{C} \mathrm{NMR}\left(\mathrm{CDCl}_{3}, 125\right.$ $\mathrm{MHz}): \delta(\mathrm{ppm}) 172.03,162.77,161.61,150.78,144.02,136.56,127.32,125.80$, $124.64,122.82,114.10,111.86,109.33,78.73,55.07,31.74,29.27,26.20$. LRMS (ESI) $[\mathrm{M}+\mathrm{H}]^{+}$found $\mathrm{m} / \mathrm{z}$ 370, HRMS (ESI) calculated for $\mathrm{C}_{20} \mathrm{H}_{16} \mathrm{NO}_{4} \mathrm{NaCl}[\mathrm{M}+\mathrm{Na}]^{+}$: 392.0666, found: 392.0670 .

(R)-6-chloro-4' -(4-fluorophenyl)-1-methylspiro[indoline-3,2'-pyran]-2,6' (3'H)-dio ne (3I).

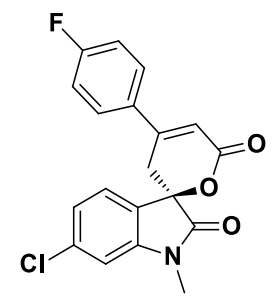

Yield 68\%; white solid; M.P.: 189.1-191.0 ${ }^{\circ} \mathrm{C} ; \mathrm{R}_{\mathrm{f}}=0.35$ (petroleum ether/ethyl acetate $=2 / 1) ;[\alpha]_{\mathrm{D}}{ }^{25}=-28.0\left(\mathrm{c}=1.0, \mathrm{CHCl}_{3}\right) ; \mathrm{HPLC}$ analysis: $74 \%$ ee [Daicel CHIRALPAK IA-H column, $20{ }^{\circ} \mathrm{C}, 254 \mathrm{~nm}$ hexane/i-PrOH = 85:15, $1.0 \mathrm{~mL} / \mathrm{min}, 254$ nm, 40.0 min (minor), 53.0 min (major)]; ${ }^{1} \mathrm{H}$ NMR $\left(\mathrm{CDCl}_{3}, 500 \mathrm{MHz}\right): \delta 7.46$ (dd, $J$ $=8.9,5.1 \mathrm{~Hz}, 2 \mathrm{H}), 7.30(\mathrm{~d}, J=8.0 \mathrm{~Hz}, 1 \mathrm{H}), 7.06(\mathrm{t}, J=8.6 \mathrm{~Hz}, 2 \mathrm{H}), 6.98(\mathrm{dd}, J=8.0$, $1.8 \mathrm{~Hz}, 1 \mathrm{H}), 6.82(\mathrm{~d}, J=1.7 \mathrm{~Hz}, 1 \mathrm{H}), 6.46(\mathrm{~s}, 1 \mathrm{H}), 3.20(\mathrm{dd}, J=17.8,1.5 \mathrm{~Hz}, 1 \mathrm{H})$, $3.14(\mathrm{~s}, 3 \mathrm{H}), 3.00(\mathrm{dd}, J=17.8,1.2 \mathrm{~Hz}, 1 \mathrm{H}) ;{ }^{13} \mathrm{C} \mathrm{NMR}\left(\mathrm{CDCl}_{3}, 125 \mathrm{MHz}\right): \delta 172.40$, $165.34,163.33,162.75,150.42,144.48,137.20,131.90,131.87,128.18,128.11$, 
$127.76,125.93,125.03,123.35,116.44,116.27,114.58,109.84,79.12,32.51,29.71$, 26.64, 14.13, 1.03. LRMS (ESI) $[\mathrm{M}+\mathrm{H}]^{+}$found m/z 358, HRMS (ESI) calculated for $\mathrm{C}_{19} \mathrm{H}_{13} \mathrm{NO}_{3} \mathrm{NaClF}[\mathrm{M}+\mathrm{Na}]^{+}:$380.0466, found: 380.0470 .

(R)-4' -(4-bromophenyl)-6-chloro-1-methylspiro[indoline-3,2'-pyran]-2,6' (3'H)-di one $(3 \mathrm{~m})$.

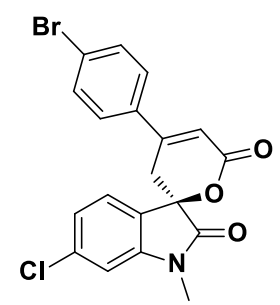

Yield 35\%; yellow solid; M.P.: 215.2-216.8 ${ }^{\circ} \mathrm{C} ; \mathrm{R}_{\mathrm{f}}=0.32$ (petroleum ether/ethyl acetate $=2 / 1) ;[\alpha]_{\mathrm{D}}{ }^{25}=-37.7\left(\mathrm{c}=1.0, \mathrm{CHCl}_{3}\right) ;$ HPLC analysis: $72 \%$ ee [Daicel CHIRALPAK IA-H column, $20{ }^{\circ} \mathrm{C}, 254 \mathrm{~nm}$ hexane/i-PrOH = 85:15, $1.0 \mathrm{~mL} / \mathrm{min}, 254$ $\mathrm{nm}, 46.2 \mathrm{~min}$ (minor), $61.9 \mathrm{~min}$ (major)]; ${ }^{1} \mathrm{H} \mathrm{NMR}\left(\mathrm{CDCl}_{3}, 500 \mathrm{MHz}\right): \delta 7.59$ (d, $J=$ $8.6 \mathrm{~Hz}, 2 \mathrm{H}), 7.41(\mathrm{~d}, J=8.6 \mathrm{~Hz}, 2 \mathrm{H}), 7.38(\mathrm{~d}, J=8.0 \mathrm{~Hz}, 1 \mathrm{H}), 7.05(\mathrm{dd}, J=8.0,1.7$ $\mathrm{Hz}, 1 \mathrm{H}), 6.90(\mathrm{~d}, J=1.7 \mathrm{~Hz}, 1 \mathrm{H}), 6.58(\mathrm{~s}, 1 \mathrm{H}), 3.25(\mathrm{dd}, J=17.8,1.2 \mathrm{~Hz}, 1 \mathrm{H}), 3.21$ (s, 3H), $3.08(\mathrm{dd}, J=17.8,1.2 \mathrm{~Hz}, 1 \mathrm{H}) ;{ }^{13} \mathrm{C} \mathrm{NMR}\left(\mathrm{CDCl}_{3}, 125 \mathrm{MHz}\right): \delta 171.93$, $162.22,149.93,144.03,136.79,134.17,131.96,127.08,125.39,125.17,124.59$, 122.93, 114.69, 109.42, 78.69, 31.89, 29.27, 26.21. LRMS (ESI) $[\mathrm{M}+\mathrm{H}]^{+}$found $\mathrm{m} / \mathrm{z}$ 418, HRMS (ESI) calculated for $\mathrm{C}_{19} \mathrm{H}_{13} \mathrm{NO}_{3} \mathrm{NaClBr}[\mathrm{M}+\mathrm{Na}]^{+}:$439.9665, found: 439.9675, 439.9648.

(R)-6-chloro-1,4'-dimethylspiro[indoline-3,2'-pyran]-2,6' (3'H)-dione (3n).

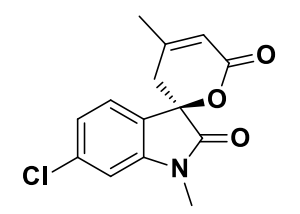

Yield 77\%; yellow solid; M.P.: 213.3-214.9 ${ }^{\circ} \mathrm{C} ; \mathrm{R}_{\mathrm{f}}=0.35$ (petroleum ether/ethyl acetate $=2 / 1) ;[\alpha]_{\mathrm{D}}^{25}=7.2\left(\mathrm{c}=1.0, \mathrm{CHCl}_{3}\right) ; \mathrm{HPLC}$ analysis: $36 \%$ ee [Daicel CHIRALPAK OD-H column, $20{ }^{\circ} \mathrm{C}, 254 \mathrm{~nm}$ hexane/i-PrOH $=80: 20,1.0 \mathrm{~mL} / \mathrm{min}$, $254 \mathrm{~nm}, 26.6 \mathrm{~min}$ (major), 92,0 $\min$ (minor)] ${ }^{1} \mathrm{H} \mathrm{NMR}\left(\mathrm{CDCl}_{3}, 500 \mathrm{MHz}\right): \delta 7.34$ (d, $J=8.0 \mathrm{~Hz}, 1 \mathrm{H}), 7.05(\mathrm{dd}, J=8.0,1.8 \mathrm{~Hz}, 1 \mathrm{H}), 6.87(\mathrm{~d}, J=1.8 \mathrm{~Hz}, 1 \mathrm{H}), 6.06(\mathrm{~s}, 1 \mathrm{H})$, 
$3.19(\mathrm{~s}, 3 \mathrm{H}), 2.84(\mathrm{~d}, J=18.2 \mathrm{~Hz}, 1 \mathrm{H}), 2.60(\mathrm{~d}, J=18.2 \mathrm{~Hz}, 1 \mathrm{H}), 2.06(\mathrm{~s}, 3 \mathrm{H}) ;{ }^{13} \mathrm{C}$ NMR $\left(\mathrm{CDCl}_{3}, 125 \mathrm{MHz}\right): \delta 172.12,161.92,153.63,143.99,136.54,125.74,124.46$, $122.80,116.11,109.28,78.68,34.61,29.26,26.12,22.80$. LRMS (ESI) $[\mathrm{M}+\mathrm{H}]^{+}$ found $\mathrm{m} / \mathrm{z}$ 278, HRMS (ESI) calculated for $\mathrm{C}_{14} \mathrm{H}_{12} \mathrm{NO}_{3} \mathrm{NaCl}[\mathrm{M}+\mathrm{Na}]^{+}$: 300.0403, found: 300.0410 .

(R)-6-bromo-4' -(4-bromophenyl)-1-methylspiro[indoline-3,2'-pyran]-2,6'(3'H)-di one (3o).

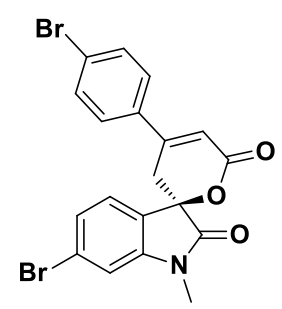

Yield 65\%; yellow solid; M.P.: $223.8-225.2{ }^{\circ} \mathrm{C} ; \mathrm{R}_{\mathrm{f}}=0.34$ (petroleum ether/ethyl acetate $=2 / 1) ;[\alpha]_{\mathrm{D}}{ }^{25}=-47.6\left(\mathrm{c}=1.0, \mathrm{CHCl}_{3}\right)$; HPLC analysis: $64 \%$ ee [Daicel CHIRALPAK IA-H column, $20{ }^{\circ} \mathrm{C}, 254 \mathrm{~nm}$ hexane/i-PrOH = 80:20, $1.0 \mathrm{~mL} / \mathrm{min}, 254$ nm, $40.0 \mathrm{~min}$ (minor), $57.2 \mathrm{~min}$ (major)] ${ }^{1} \mathrm{H} \mathrm{NMR}\left(\mathrm{CDCl}_{3}, 500 \mathrm{MHz}\right): \delta 7.58$ (d, $J=$ $8.2 \mathrm{~Hz}, 2 \mathrm{H}), 7.40(\mathrm{~d}, J=8.2 \mathrm{~Hz}, 2 \mathrm{H}), 7.32(\mathrm{~d}, J=7.9 \mathrm{~Hz}, 1 \mathrm{H}), 7.22(\mathrm{~d}, J=7.9 \mathrm{~Hz}$, 1H), $7.05(\mathrm{~s}, 1 \mathrm{H}), 6.57(\mathrm{~s}, 1 \mathrm{H}), 3.26(\mathrm{dd}, J=17.8,1.1 \mathrm{~Hz} 1 \mathrm{H}), 3.21(\mathrm{~s}, 3 \mathrm{H}), 3.08(\mathrm{dd}, J$ $=17.8,1.1 \mathrm{~Hz}, 1 \mathrm{H}) ;{ }^{13} \mathrm{C} \mathrm{NMR}\left(\mathrm{CDCl}_{3}, 125 \mathrm{MHz}\right): \delta 171.82,162.20,149.92,144.06$, $134.15,131.96,127.09,125.92,125.17,124.87,124.66,114.67,112.22,78.74,31.83$, 29.27, 26.21. LRMS (ESI) $[\mathrm{M}+\mathrm{H}]^{+}$found $\mathrm{m} / \mathrm{z}$ 462, HRMS (ESI) calculated for $\mathrm{C}_{19} \mathrm{H}_{13} \mathrm{NO}_{3} \mathrm{NaBr}_{2}[\mathrm{M}+\mathrm{Na}]^{+}:$483.9160, found: 483.9179, 485.9151, 487.9140.

(R)-6-bromo-4' -(4-methoxyphenyl)-1-methylspiro[indoline-3,2'-pyran]-2,6'(3'H)dione (3p).

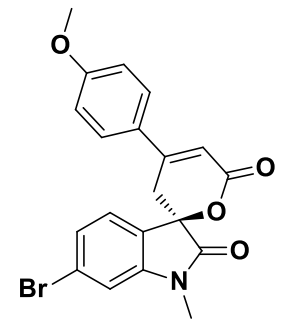

Yield 55\%; yellow solid; M.P.: 212.1-213.8 ${ }^{\circ} \mathrm{C} ; \mathrm{R}_{\mathrm{f}}=0.33$ (petroleum ether/ethyl 
acetate $=2 / 1) ;[\alpha]_{\mathrm{D}}^{25}=-42.0\left(\mathrm{c}=1.0, \mathrm{CHCl}_{3}\right) ;$ HPLC analysis: $98 \%$ ee [Daicel CHIRALPAK AD-H column, $20{ }^{\circ} \mathrm{C}, 254 \mathrm{~nm}$ hexane/i-PrOH $=85: 15,1.0 \mathrm{~mL} / \mathrm{min}$, $254 \mathrm{~nm}, 61.0 \mathrm{~min}$ (minor), $84.1 \mathrm{~min}$ (major)] ${ }^{1} \mathrm{H} \mathrm{NMR}\left(\mathrm{CDCl}_{3}, 500 \mathrm{MHz}\right): \delta 7.51$ (d, $J=8.9 \mathrm{~Hz}, 2 \mathrm{H}), 7.33(\mathrm{~d}, J=8.0 \mathrm{~Hz}, 1 \mathrm{H}), 7.19(\mathrm{dd}, J=8.0,1.6 \mathrm{~Hz}, 1 \mathrm{H}), 7.04(\mathrm{~d}, J=$ $1.6 \mathrm{~Hz}, 1 \mathrm{H}), 6.95(\mathrm{~d}, J=8.9 \mathrm{~Hz}, 2 \mathrm{H}), 6.52(\mathrm{~s}, 1 \mathrm{H}), 3.86(\mathrm{~s}, 3 \mathrm{H}), 3.32(\mathrm{dd}, J=17.7$, $1.6 \mathrm{~Hz}, 1 \mathrm{H}), 3.22$ (s, 3H), 3.08-2.99 (dd, $J=17.7,1.6 \mathrm{~Hz}, 1 \mathrm{H}) ;{ }^{13} \mathrm{C} \mathrm{NMR}\left(\mathrm{CDCl}_{3}\right.$, $125 \mathrm{MHz}$ ): $\delta$ 171.93, 162.74, 161.62, 150.76, 144.06, 127.32, 126.34, 125.80, 124.91, 124.44, 114.10, 112.12, 111.85, 78.77, 55.07, 31.68, 29.27, 26.19. LRMS (ESI) $[\mathrm{M}+\mathrm{H}]^{+}$found $\mathrm{m} / \mathrm{z}$ 414, HRMS (ESI) calculated for $\mathrm{C}_{20} \mathrm{H}_{16} \mathrm{NO}_{4} \mathrm{NaBr}[\mathrm{M}+\mathrm{Na}]^{+}$: 436.0160, found: 436.0172, 438.0143 .

(R)-6-methoxy-4' -(4-methoxyphenyl)-1-methylspiro[indoline-3,2'-pyran]-2,6' (3' H )-dione (3q).

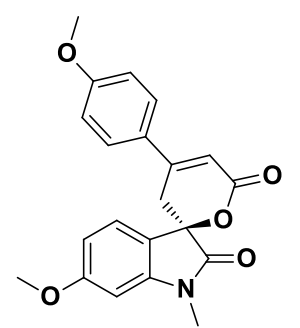

Yield 47\%; white solid; M.P.: $189.1-190.8{ }^{\circ} \mathrm{C} ; \mathrm{R}_{\mathrm{f}}=0.31$ (petroleum ether/ethyl acetate $=2 / 1) ;[\alpha]_{\mathrm{D}}^{25}=-51.8\left(\mathrm{c}=1.0, \mathrm{CHCl}_{3}\right) ;$ HPLC analysis: $84 \%$ ee [Daicel CHIRALPAK OD-H column, $20{ }^{\circ} \mathrm{C}, 254 \mathrm{~nm}$ hexane/i-PrOH $=80: 20,1.0 \mathrm{~mL} / \mathrm{min}$, $254 \mathrm{~nm}, 85.6 \mathrm{~min}$ (major), $135.6 \mathrm{~min}$ (minor)] ${ }^{1} \mathrm{H} \mathrm{NMR}\left(\mathrm{CDCl}_{3}, 500 \mathrm{MHz}\right): \delta 7.53$ (d, $J=8.9 \mathrm{~Hz}, 2 \mathrm{H}), 7.37(\mathrm{~d}, J=8.3 \mathrm{~Hz}, 1 \mathrm{H}), 6.96(\mathrm{~d}, J=8.9 \mathrm{~Hz}, 2 \mathrm{H}), 6.51(\mathrm{dd}, J=8.6$, $1.9 \mathrm{~Hz}, 2 \mathrm{H}), 6.44$ (d, $J=2.2 \mathrm{~Hz}, 1 \mathrm{H}), 3.86$ (s, 3H), 3.84 (s, 3H), 3.34 (dd, $J=17.6$, $1.7 \mathrm{~Hz}, 1 \mathrm{H}), 3.22$ (s, 3H), 3.08-3.00 (dd, $J=17.6,1.7 \mathrm{~Hz}, 1 \mathrm{H}) ;{ }^{13} \mathrm{C} \mathrm{NMR}\left(\mathrm{CDCl}_{3}\right.$, $125 \mathrm{MHz}): \delta 172.98,163.73,162.22,161.89,151.47,144.77,128.06,127.71,125.07$, 119.92, 114.45, 112.46, 106.76, 96.94, 79.51, 55.61, 55.46, 32.45, 29.68, 26.46. LRMS (ESI) $[\mathrm{M}+\mathrm{H}]^{+}$found $\mathrm{m} / \mathrm{z}$ 366, HRMS (ESI) calculated for $\mathrm{C}_{21} \mathrm{H}_{19} \mathrm{NO}_{5} \mathrm{Na}$ $[\mathrm{M}+\mathrm{H}]^{+}:$388.1161, found: 388.1159 . 
dione (3r).

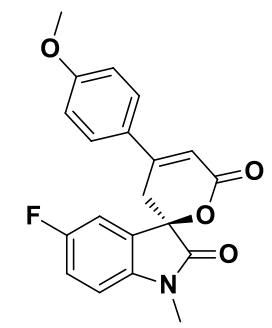

Yield 59\%; yellow solid; M.P.: 204.1-206.7 ${ }^{\circ} \mathrm{C} ; \mathrm{R}_{\mathrm{f}}=0.37$ (petroleum ether/ethyl acetate $=2 / 1) ;[\alpha]_{\mathrm{D}}{ }^{25}=-26.3\left(\mathrm{c}=1.0, \mathrm{CHCl}_{3}\right) ; \mathrm{HPLC}$ analysis: $77 \%$ ee [Daicel CHIRALPAK OD-H column, $20{ }^{\circ} \mathrm{C}, 254 \mathrm{~nm}$ hexane/i-PrOH $=80: 20,1.0 \mathrm{~mL} / \mathrm{min}$, $254 \mathrm{~nm}, 51.1 \mathrm{~min}$ (major), $86.6 \mathrm{~min}$ (minor)] ${ }^{1} \mathrm{H} \mathrm{NMR}\left(\mathrm{CDCl}_{3}, 400 \mathrm{MHz}\right): \delta 7.53$ (d, $J=8.9 \mathrm{~Hz}, 2 \mathrm{H}), 7.22(\mathrm{dd}, J=7.6,2.6 \mathrm{~Hz}, 1 \mathrm{H}), 7.10(\mathrm{td}, J=8.7,2.6 \mathrm{~Hz}, 1 \mathrm{H}), 6.97(\mathrm{~d}$, $J=8.9 \mathrm{~Hz}, 2 \mathrm{H}), 6.83(\mathrm{dd}, J=8.7,4.0 \mathrm{~Hz}, 1 \mathrm{H}), 6.53(\mathrm{~s}, 1 \mathrm{H}), 3.87(\mathrm{~s}, 3 \mathrm{H}), 3.37(\mathrm{dd}, J$ $=17.7,1.6 \mathrm{~Hz}, 1 \mathrm{H}), 3.23(\mathrm{~s}, 3 \mathrm{H}), 3.05(\mathrm{dd}, J=17.7,1.6 \mathrm{~Hz}, 1 \mathrm{H}) ;{ }^{13} \mathrm{C} \mathrm{NMR}\left(\mathrm{CDCl}_{3}\right.$, $125 \mathrm{MHz}): \delta 172.28,163.09,162.09,160.19,158.26,151.27,139.14,129.17,127.78$, 127.72, 117.41, 117.22, 114.55, 112.50, 112.30, 112.19, 109.75, 109.68, 79.57, 55.51, 32.10, 29.70, 26.65. LRMS (ESI) $[\mathrm{M}+\mathrm{H}]^{+}$found m/z 354, HRMS (ESI) calculated for $\mathrm{C}_{20} \mathrm{H}_{16} \mathrm{NO}_{4} \mathrm{NaF}[\mathrm{M}+\mathrm{Na}]^{+}:$376.0961, found: 376.0954 .

(R)-5-chloro-4' -(4-methoxyphenyl)-1-methylspiro[indoline-3,2'-pyran]-2,6' (3'H)dione (3s).

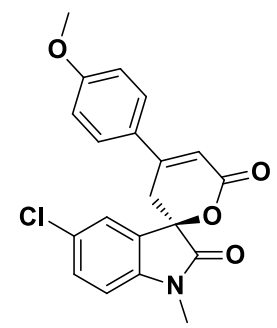

Yield 62\%; yellow solid; M.P.: $185.6-187.3{ }^{\circ} \mathrm{C} ; \mathrm{R}_{\mathrm{f}}=0.38$ (petroleum ether/ethyl acetate $=2 / 1) ;[\alpha]_{\mathrm{D}}^{25}=-78.2\left(\mathrm{c}=1.0, \mathrm{CHCl}_{3}\right) ;$ HPLC analysis: $84 \%$ ee [Daicel CHIRALPAK OD-H column, $20{ }^{\circ} \mathrm{C}, 254 \mathrm{~nm}$ hexane/i-PrOH $=80: 20,1.0 \mathrm{~mL} / \mathrm{min}$, $254 \mathrm{~nm}, 56.4 \mathrm{~min}$ (major), $108.8 \mathrm{~min}$ (minor)] ${ }^{1} \mathrm{H} \mathrm{NMR}\left(\mathrm{CDCl}_{3}, 500 \mathrm{MHz}\right): \delta 7.54$ (d, $J=8.9 \mathrm{~Hz}, 2 \mathrm{H}), 7.45(\mathrm{~d}, J=2.1 \mathrm{~Hz}, 1 \mathrm{H}), 7.38(\mathrm{dd}, J=8.3,2.1 \mathrm{~Hz}, 1 \mathrm{H}), 6.97(\mathrm{~d}, J=$ $8.9 \mathrm{~Hz}, 2 \mathrm{H}), 6.83(\mathrm{~d}, J=8.3 \mathrm{~Hz}, 1 \mathrm{H}), 6.54(\mathrm{~s}, 1 \mathrm{H}), 3.87$ (s, 3H), 3.35 (dd, $J=17.7$, $1.3 \mathrm{~Hz}, 1 \mathrm{H}), 3.23(\mathrm{~s}, 3 \mathrm{H}), 3.06(\mathrm{dd}, J=17.7,1.3 \mathrm{~Hz}, 1 \mathrm{H}) ;{ }^{13} \mathrm{C} \mathrm{NMR}\left(\mathrm{CDCl}_{3}, 125\right.$ 
MHz): $\delta 172.11,163.01,162.07,151.15,141.72,130.91,129.39,128.77,127.76$, $127.70,124.63,114.54,112.21,109.95,79.36,55.49,32.04,29.68,26.62$. LRMS (ESI) $[\mathrm{M}+\mathrm{H}]^{+}$found $\mathrm{m} / \mathrm{z} 370$, HRMS (ESI) calculated for $\mathrm{C}_{20} \mathrm{H}_{16} \mathrm{NO}_{4} \mathrm{NaCl}[\mathrm{M}+\mathrm{Na}]^{+}$: 392.0666, found: 392.0668 .

(R)-4-chloro-4' -(4-methoxyphenyl)-1-methylspiro[indoline-3,2'-pyran]-2,6' (3'H)dione (3t).

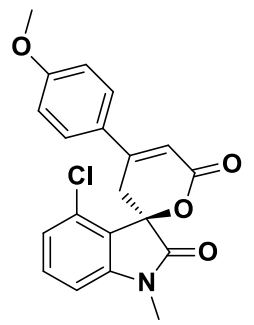

Yield 59\%; yellow solid; M.P.: $175.5-177.3{ }^{\circ} \mathrm{C} ; \mathrm{R}_{\mathrm{f}}=0.33$ (petroleum ether/ethyl acetate $=2 / 1) ;[\alpha]_{\mathrm{D}}^{25}=-42.3\left(\mathrm{c}=1.0, \mathrm{CHCl}_{3}\right)$; HPLC analysis: $>99 \%$ ee [Daicel CHIRALPAKOD-H column, $20{ }^{\circ} \mathrm{C}, 254 \mathrm{~nm}$ hexane $/ \mathrm{i}-\mathrm{PrOH}=80: 20,1.0 \mathrm{~mL} / \mathrm{min}$, $254 \mathrm{~nm}, 39.7 \mathrm{~min}$ (major), $99.0 \mathrm{~min}$ (minor)] ${ }^{1} \mathrm{H} \mathrm{NMR}\left(\mathrm{CDCl}_{3}, 500 \mathrm{MHz}\right): \delta 7.53$ (d, $J=8.9 \mathrm{~Hz}, 2 \mathrm{H}), 7.36(\mathrm{t}, J=8.1 \mathrm{~Hz}, 1 \mathrm{H}), 7.10(\mathrm{~d}, J=8.3 \mathrm{~Hz}, 1 \mathrm{H}), 6.94(\mathrm{~d}, J=8.9 \mathrm{~Hz}$, 2H), $6.79(\mathrm{~d}, J=7.8 \mathrm{~Hz}, 1 \mathrm{H}), 6.49(\mathrm{~d}, J=2.4 \mathrm{~Hz}, 1 \mathrm{H}), 3.88(\mathrm{dd}, J=18.1,1.5 \mathrm{~Hz}, 1 \mathrm{H})$, $3.85(\mathrm{~d}, J=3.4 \mathrm{~Hz}, 3 \mathrm{H}), 3.16(\mathrm{~s}, 3 \mathrm{H}), 2.92(\mathrm{dd}, J=18.1,1.5 \mathrm{~Hz}, 1 \mathrm{H}) ;{ }^{13} \mathrm{C}$ $\mathrm{NMR}\left(\mathrm{CDCl}_{3}, 125 \mathrm{MHz}\right): \delta 172.91,163.60,161.73,149.83,145.08,132.24,132.13$, 128.07, 127.64, 124.64, 123.58, 114.32, 111.99, 107.26, 79.71, 55.44, 29.68, 29.13, 26.52.LRMS (ESI) $[\mathrm{M}+\mathrm{H}]^{+}$found $\mathrm{m} / \mathrm{z}$ 370, HRMS (ESI) calculated for $\mathrm{C}_{20} \mathrm{H}_{16} \mathrm{NO}_{4} \mathrm{NaCl}[\mathrm{M}+\mathrm{Na}]^{+}$: 392.0666, found: 392.0669 .

(R)-5-bromo-4' -(4-methoxyphenyl)-1-methylspiro[indoline-3,2'-pyran]-2,6'(3'H)dione (3u).

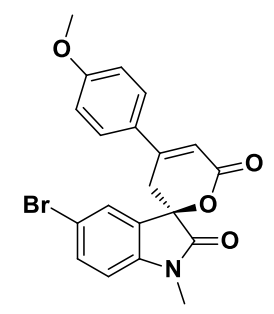

Yield 54\%; yellow solid; M.P.: $205.5-207.2{ }^{\circ} \mathrm{C} ; \mathrm{R}_{\mathrm{f}}=0.36$ (petroleum ether/ethyl 
acetate $=2 / 1) ;[\alpha]_{\mathrm{D}}^{25}=-98.9\left(\mathrm{c}=1.0, \mathrm{CHCl}_{3}\right) ;$ HPLC analysis: $58 \%$ ee [Daicel CHIRALPAK OD-H column, $20{ }^{\circ} \mathrm{C}, 254 \mathrm{~nm}$ hexane/i-PrOH $=80: 20,1.0 \mathrm{~mL} / \mathrm{min}$, $254 \mathrm{~nm}, 55.8 \mathrm{~min}$ (major), $117.5 \mathrm{~min}$ (minor)] ${ }^{1} \mathrm{H} \mathrm{NMR}\left(\mathrm{CDCl}_{3}, 400 \mathrm{MHz}\right): \delta 7.59$ (d, $J=1.8 \mathrm{~Hz}, 1 \mathrm{H}), 7.57-7.51(\mathrm{~m}, 3 \mathrm{H}), 6.98(\mathrm{~d}, J=8.8 \mathrm{~Hz}, 2 \mathrm{H}), 6.80(\mathrm{~d}, 1 \mathrm{H}), 6.55(\mathrm{~d}, J$ $=1.6 \mathrm{~Hz}, 1 \mathrm{H}), 3.88(\mathrm{~s}, 3 \mathrm{H}), 3.36(\mathrm{dd}, J=17.6,1.6 \mathrm{~Hz}, 1 \mathrm{H}), 3.24(\mathrm{~s}, 3 \mathrm{H}), 3.07(\mathrm{dd}, J$ $=17.6,1.6 \mathrm{~Hz}, 1 \mathrm{H}) ;{ }^{13} \mathrm{C} \mathrm{NMR}\left(\mathrm{CDCl}_{3}, 125 \mathrm{MHz}\right): \delta$ 172.06, 163.07, 162.10, 151.16, $142.24,133.88,129.76,127.79,127.72,127.38,115.98,114.57,112.23,110.44$, 79.33, 55.51, 32.08, 29.71, 26.62. LRMS (ESI) $[\mathrm{M}+\mathrm{H}]^{+}$found m/z 414, HRMS (ESI) calculated for $\mathrm{C}_{20} \mathrm{H}_{16} \mathrm{NO}_{4} \mathrm{NaBr}[\mathrm{M}+\mathrm{Na}]^{+}$: 436.0160, found: 436.0151, 438.0119.

(R)-5-methoxy-4' -(4-methoxyphenyl)-1-methylspiro[indoline-3,2'-pyran]-2,6' (3'H )-dione (3v).

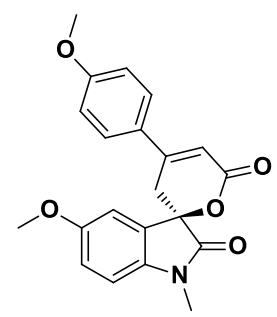

Yield 80\%; white solid; M.P.: $150.4-152.3{ }^{\circ} \mathrm{C} ; \mathrm{R}_{\mathrm{f}}=0.38$ (petroleum ether/ethyl acetate $=2 / 1) ;[\alpha]_{\mathrm{D}}^{25}=-75.4\left(\mathrm{c}=1.0, \mathrm{CHCl}_{3}\right) ;$ HPLC analysis: $71 \%$ ee [Daicel CHIRALPAK OD-H column, $20{ }^{\circ} \mathrm{C}, 254 \mathrm{~nm}$ hexane/i-PrOH $=80: 20,1.0 \mathrm{~mL} / \mathrm{min}$, $254 \mathrm{~nm}, 74.8 \mathrm{~min}$ (major), $161.7 \mathrm{~min}$ (minor)] ${ }^{1} \mathrm{H} \mathrm{NMR}\left(\mathrm{CDCl}_{3}, 500 \mathrm{MHz}\right): \delta 7.53$ (d, $J=8.9 \mathrm{~Hz}, 2 \mathrm{H}), 7.09(\mathrm{~d}, J=2.5 \mathrm{~Hz}, 1 \mathrm{H}), 6.96(\mathrm{~d}, J=8.9 \mathrm{~Hz}, 2 \mathrm{H}), 6.92-6.88(\mathrm{~m}, 1 \mathrm{H})$, $6.80(\mathrm{~d}, J=8.5 \mathrm{~Hz}, 1 \mathrm{H}), 6.53(\mathrm{~s}, 1 \mathrm{H}), 3.86(\mathrm{~s}, 3 \mathrm{H}), 3.76(\mathrm{~s}, 3 \mathrm{H}), 3.35(\mathrm{dd}, J=17.7$, $1.6 \mathrm{~Hz}, 1 \mathrm{H}), 3.21(\mathrm{~s}, 3 \mathrm{H}), 3.06(\mathrm{dd}, J=17.7,1.6 \mathrm{~Hz}, 1 \mathrm{H}) ;{ }^{13} \mathrm{C} \mathrm{NMR}\left(\mathrm{CDCl}_{3}, 125\right.$ $\mathrm{MHz}): \delta 172.30,163.43,161.92,156.34,151.28,136.42,129.03,127.96,127.71$, 114.96, 114.93, 114.46, 112.33, 111.70, 109.36, 79.89, 55.87, 55.46, 32.31, 26.55 . LRMS (ESI) $[\mathrm{M}+\mathrm{H}]^{+}$found $\mathrm{m} / \mathrm{z}$ 366, HRMS (ESI) calculated for $\mathrm{C}_{21} \mathrm{H}_{19} \mathrm{NO}_{5} \mathrm{Na}$ $[\mathrm{M}+\mathrm{H}]^{+}:$388.1161, found:388.1158.

(R)-1,4' -diphenylspiro[indoline-3,2'-pyran]-2,6' (3'H)-dione(3w). 


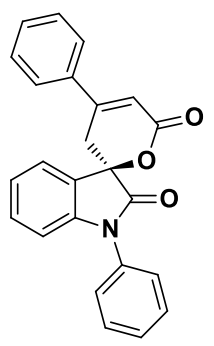

Yield 56\%; white solid; M.P.: $153.4-155.3{ }^{\circ} \mathrm{C} ; \mathrm{R}_{\mathrm{f}}=0.36$ (petroleum ether/ethyl acetate $=2 / 1) ;[\alpha]_{\mathrm{D}}^{25}=+3.8\left(\mathrm{c}=1.0, \mathrm{CHCl}_{3}\right) ;$ HPLC analysis: $74 \%$ ee [Daicel CHIRALPAK OD-H column, $20{ }^{\circ} \mathrm{C}, 254 \mathrm{~nm}$ hexane/i-PrOH $=80: 20,1.0 \mathrm{~mL} / \mathrm{min}$, $254 \mathrm{~nm}, 40.8 \mathrm{~min}$ (major), $70.9 \mathrm{~min}$ (minor)] ${ }^{1} \mathrm{H} \mathrm{NMR}\left(\mathrm{CDCl}_{3}, 500 \mathrm{MHz}\right) \delta 7.61-$ $7.50(\mathrm{~m}, 1 \mathrm{H}), 7.44(\mathrm{t}, J=8.3,2.7,1.8 \mathrm{~Hz}, 1 \mathrm{H}), 7.31(\mathrm{td}, J=7.8,1.3 \mathrm{~Hz}, 1 \mathrm{H}), 7.26(\mathrm{~s}$, $1 \mathrm{H}), 7.09(\mathrm{td}, J=7.6,0.9 \mathrm{~Hz}, 1 \mathrm{H}), 6.86(\mathrm{~d}, J=8.0 \mathrm{~Hz}, 1 \mathrm{H}), 6.62(\mathrm{t}, J=1.4 \mathrm{~Hz}, 1 \mathrm{H})$, $3.53-3.45(\mathrm{~m}, 1 \mathrm{H}), 3.24(\mathrm{dd}, J=17.8,1.2 \mathrm{~Hz}, 1 \mathrm{H}) .{ }^{1} \mathrm{H}$ NMR $\left(500 \mathrm{MHz}, \mathrm{CDCl}_{3}\right) \delta$ $7.58,7.58,7.57,7.56,7.56,7.55,7.54,7.54,7.52,7.46,7.45,7.44,7.43,7.42,7.33$, $7.32,7.31,7.31,7.30,7.29,7.11,7.09,7.07,6.87,6.85,6.61,3.50,3.50,3.47,3.47$, 3.27, 3.26, 3.23, 3.23. LRMS (ESI) $[\mathrm{M}+\mathrm{H}]^{+}$found $\mathrm{m} / \mathrm{z}$ 366, HRMS (ESI) calculated for $\mathrm{C}_{24} \mathrm{H}_{17} \mathrm{NO}_{3} \mathrm{Na}[\mathrm{M}+\mathrm{H}]^{+}: 390.1107$, found: 390.1106 . 


\section{X-ray Structure of compound 3f}
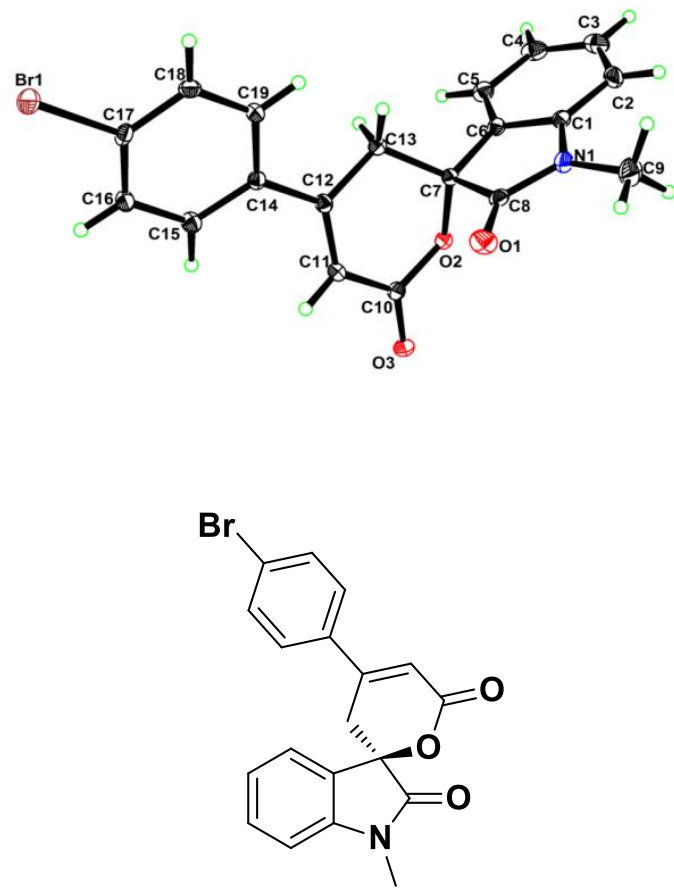

Figure S1. X-ray Structure of compound 3k

The S-configuration of (+)- 3o was determined by the X-ray analysis of its crystal (Figure S1). Thecrystal was prepared from the solution of (+)- 3o in dichloromethane/ methanol. The structure was solved and refined using the Bruker SHELXTL Software Package, The ratio of minimum to maximum apparent transmission was 0.576 . 


\section{References and notes}

a) The information of this reaction have been reported. ${ }^{1}$

b) The information of this reaction have been reported. ${ }^{2}$

[1] R. Sharma, A. K. Pandey, R. Shivahare, K. Srivastava, S. Gupta, P. M. Chauhan, Bioorg. Med. Chem. Lett 2014, 24, 298-301.

[2] J. L. Li, B. Sahoo, C. G. Daniliuc, F. Glorius, Angew. Chem. Int. Ed. 2014, 53, 10515-9. 


\section{Part III NMR Spectra}

(R)-1-methyl-4'-phenylspiro[indoline-3,2'-pyran]-2,6'(3'H)-dione (3a)

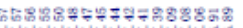

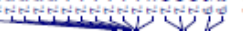

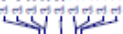
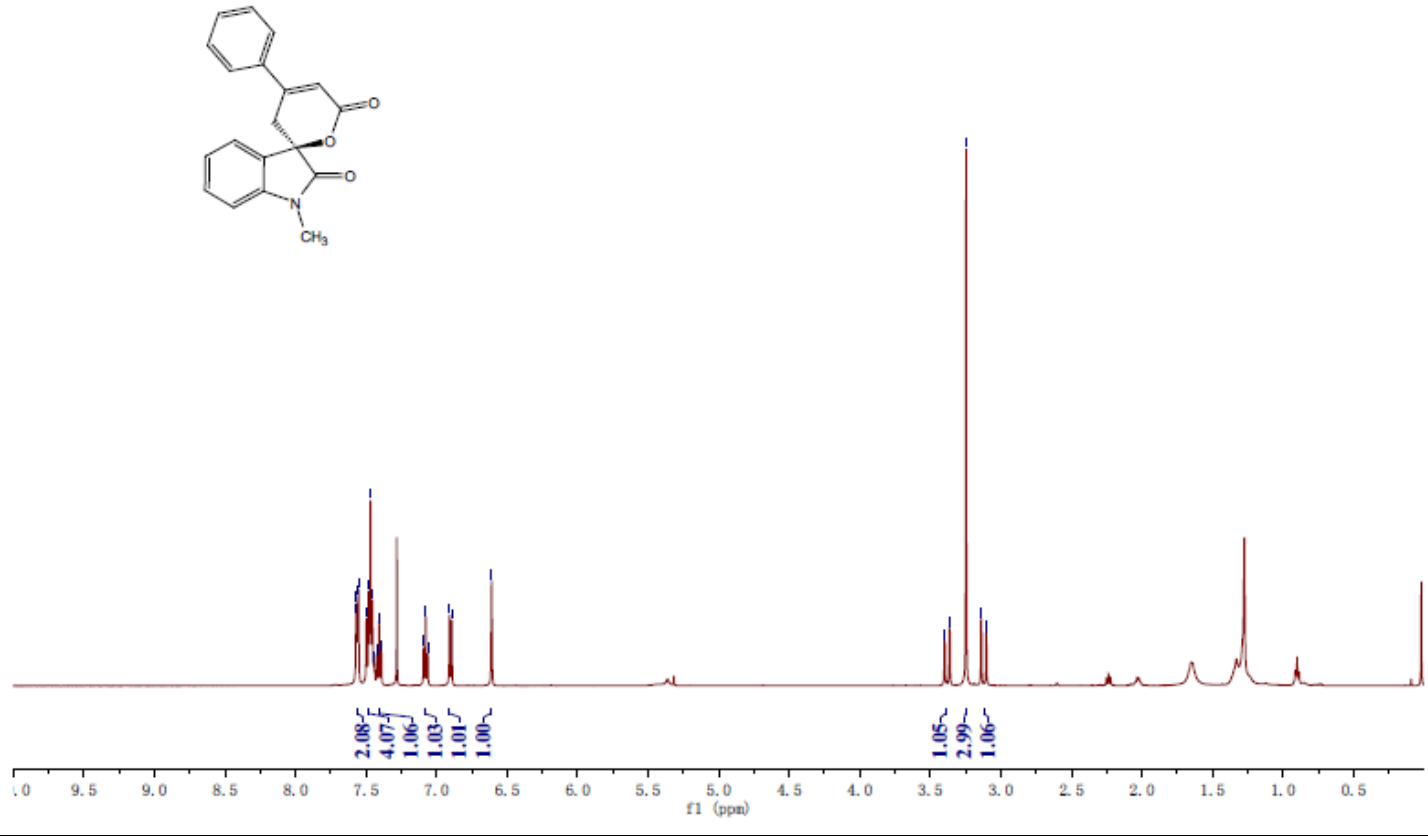

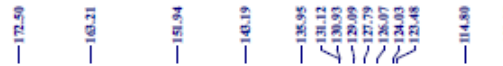
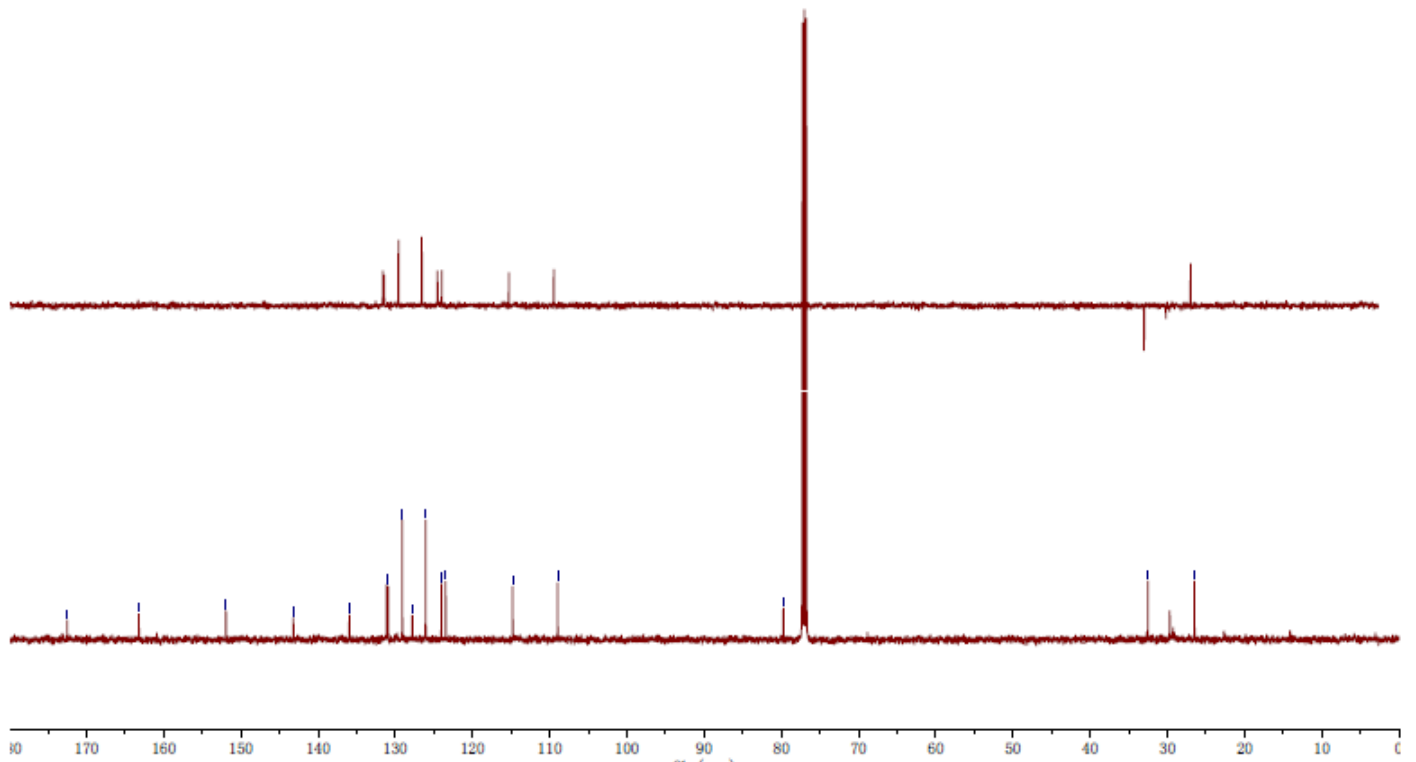


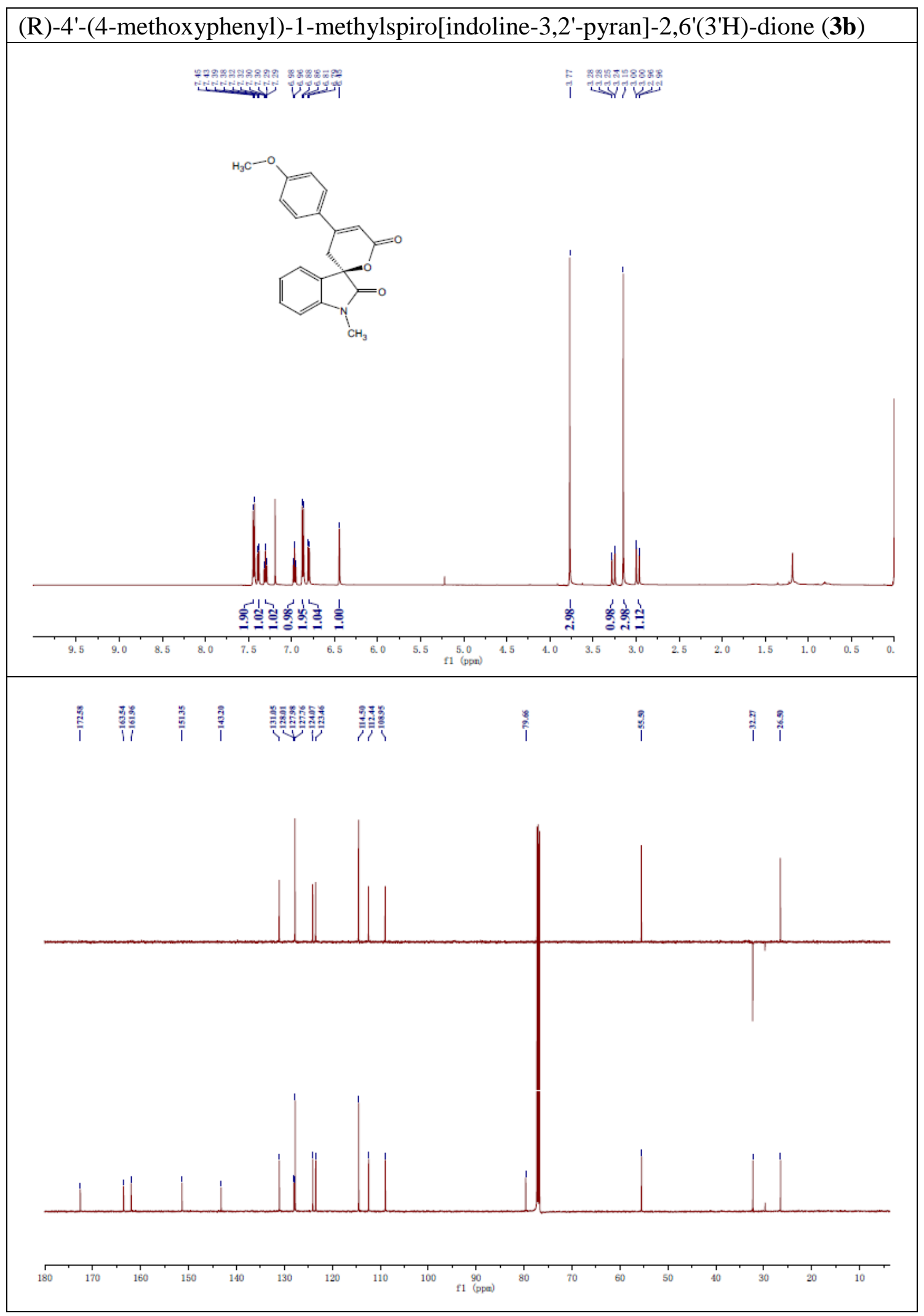




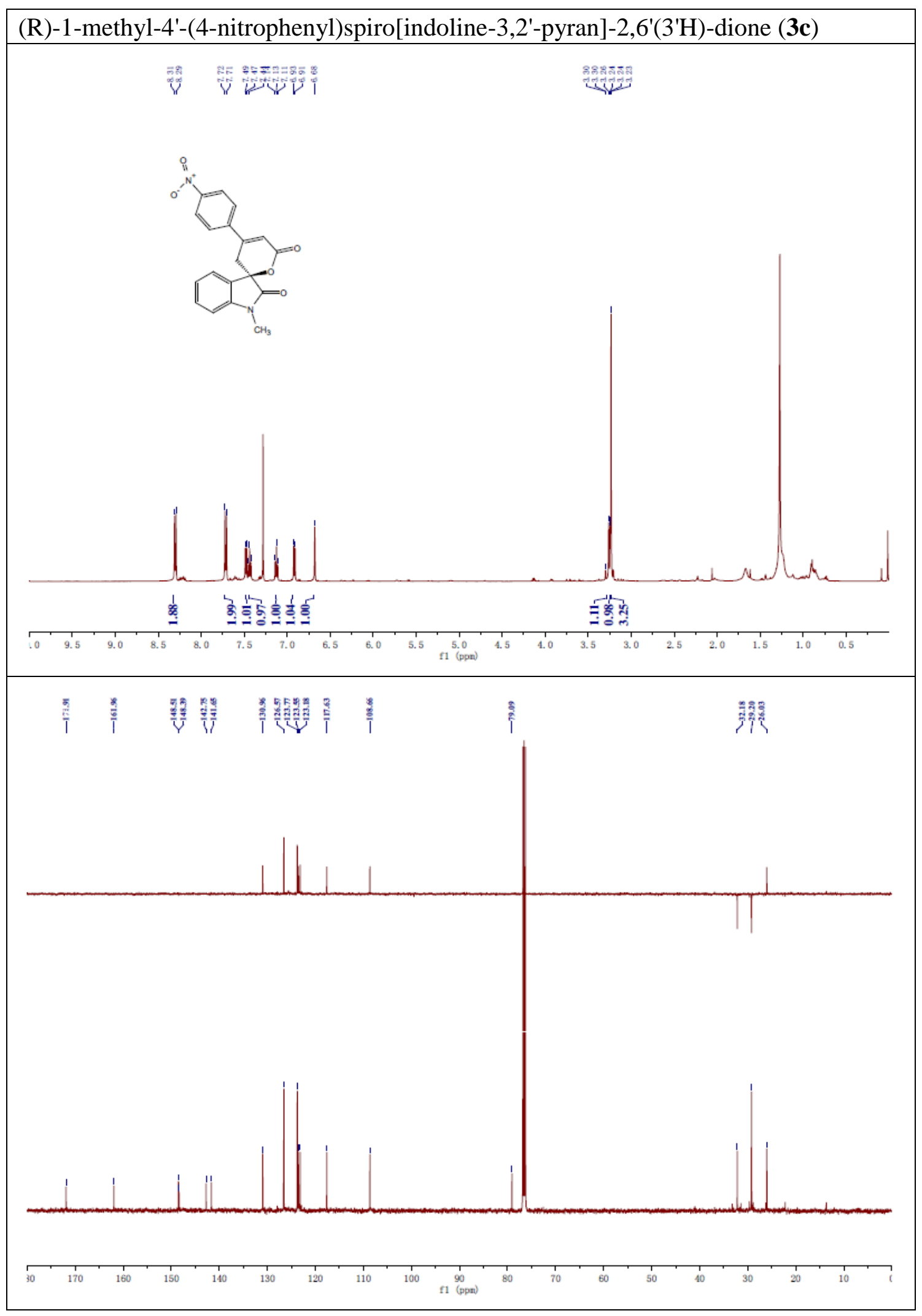




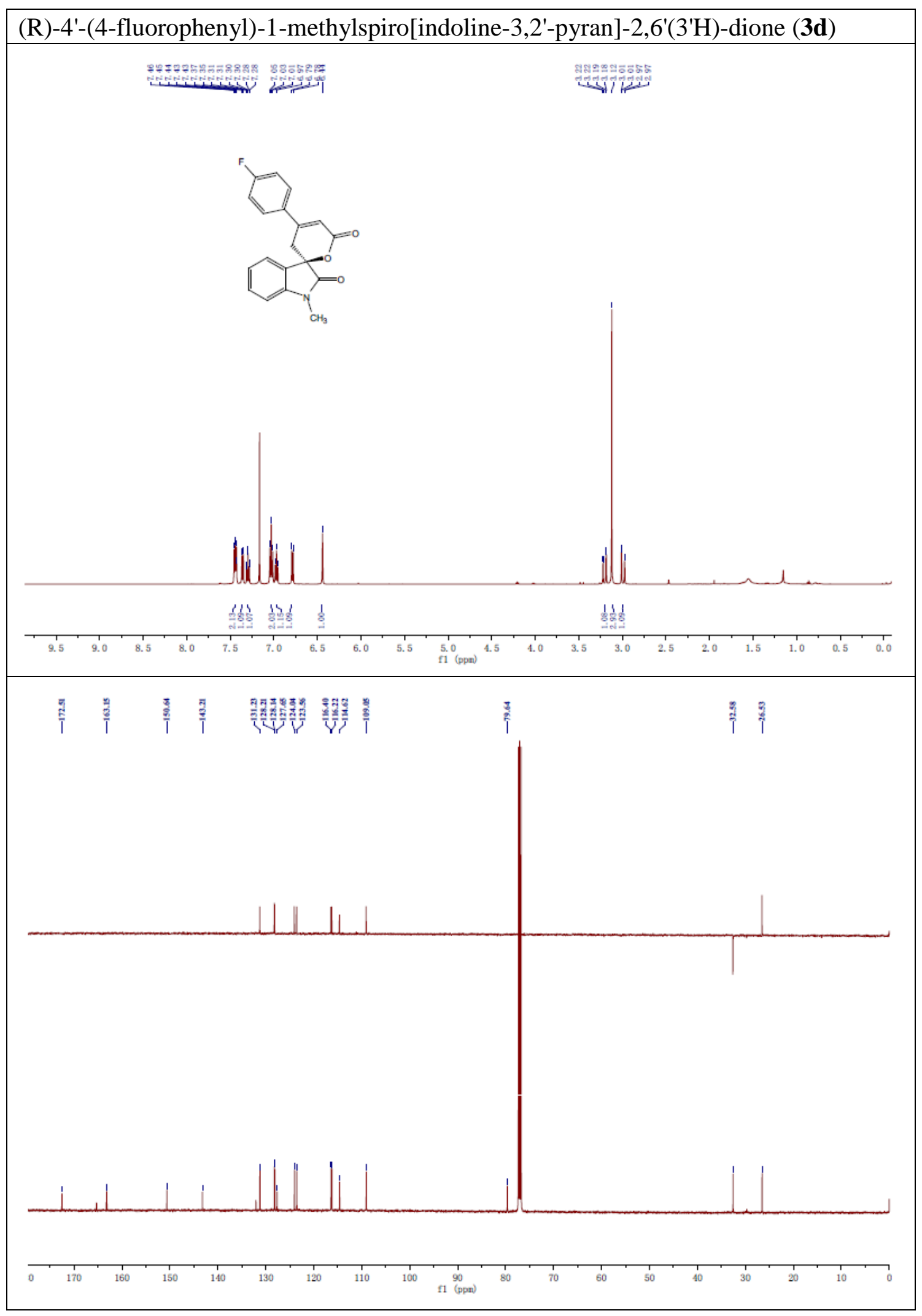


(R)-4'-(4-chlorophenyl)-1-methylspiro[indoline-3,2'-pyran]-2,6'(3'H)-dione (3e)

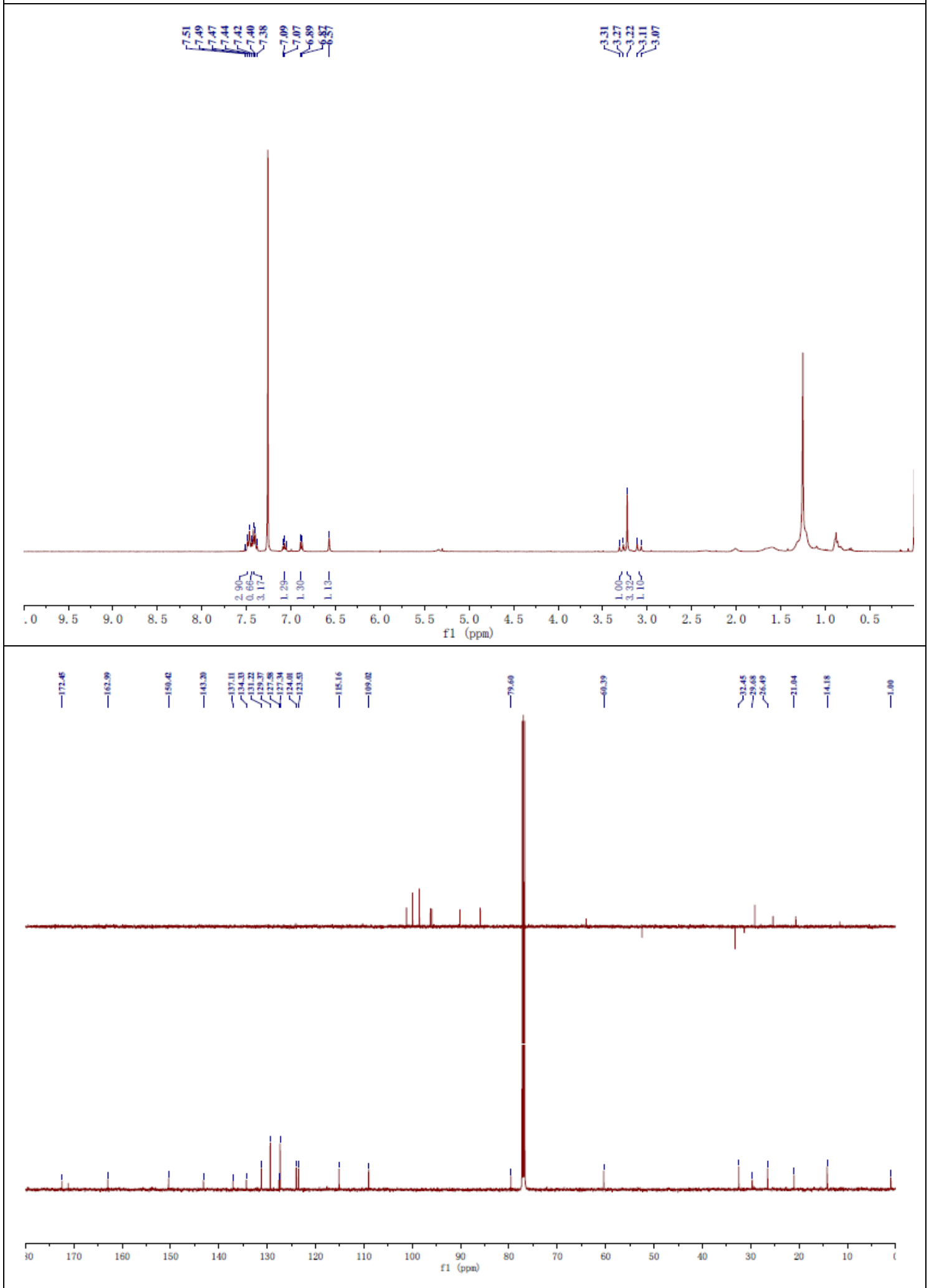




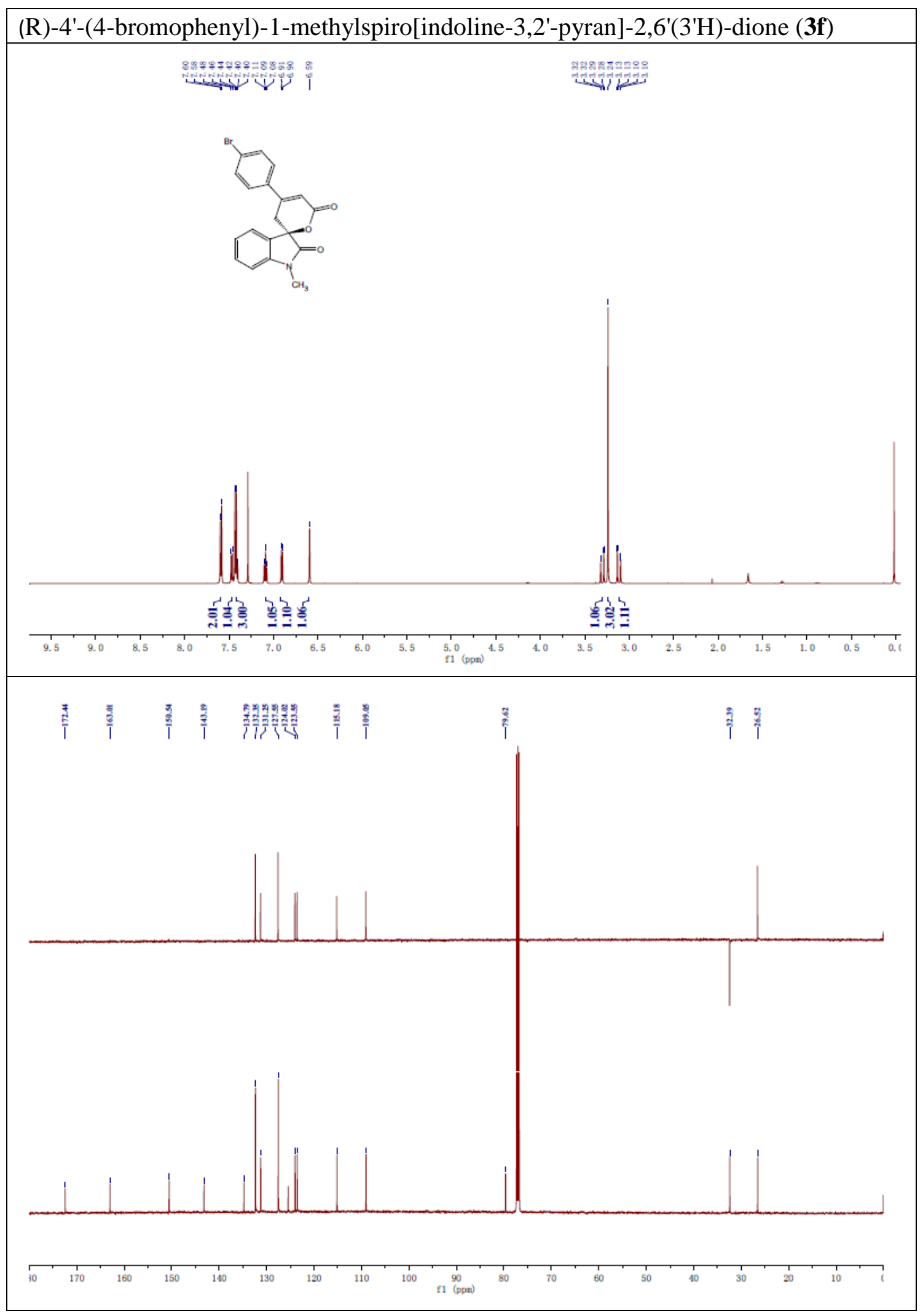


(R)-4'-(furan-2-yl)-1-methylspiro[indoline-3,2'-pyran]-2,6'(3'H)-dione (3g)

$88+4=-39$.

독ำ
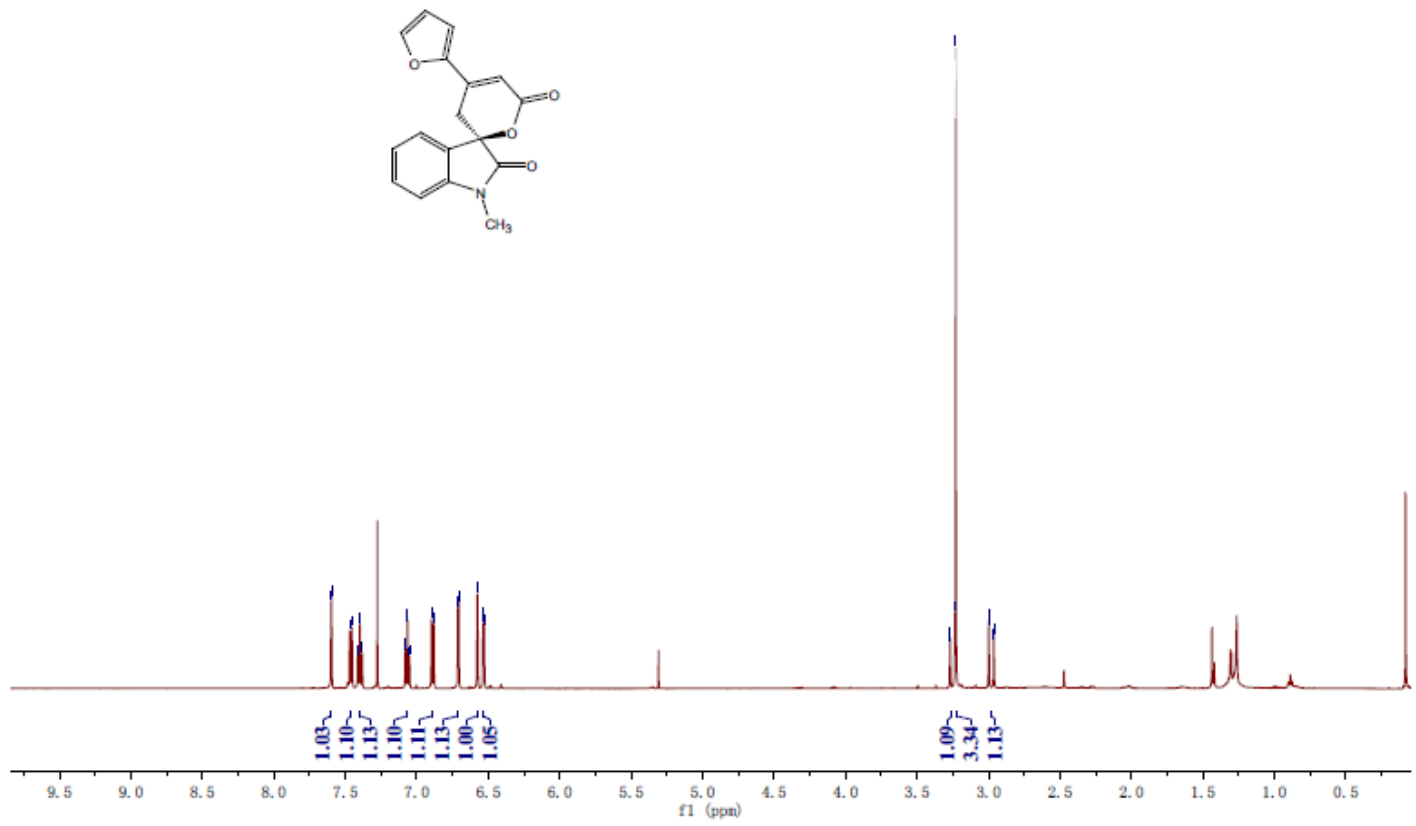

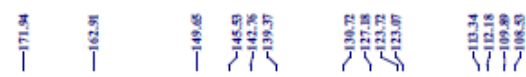

iุ

是当
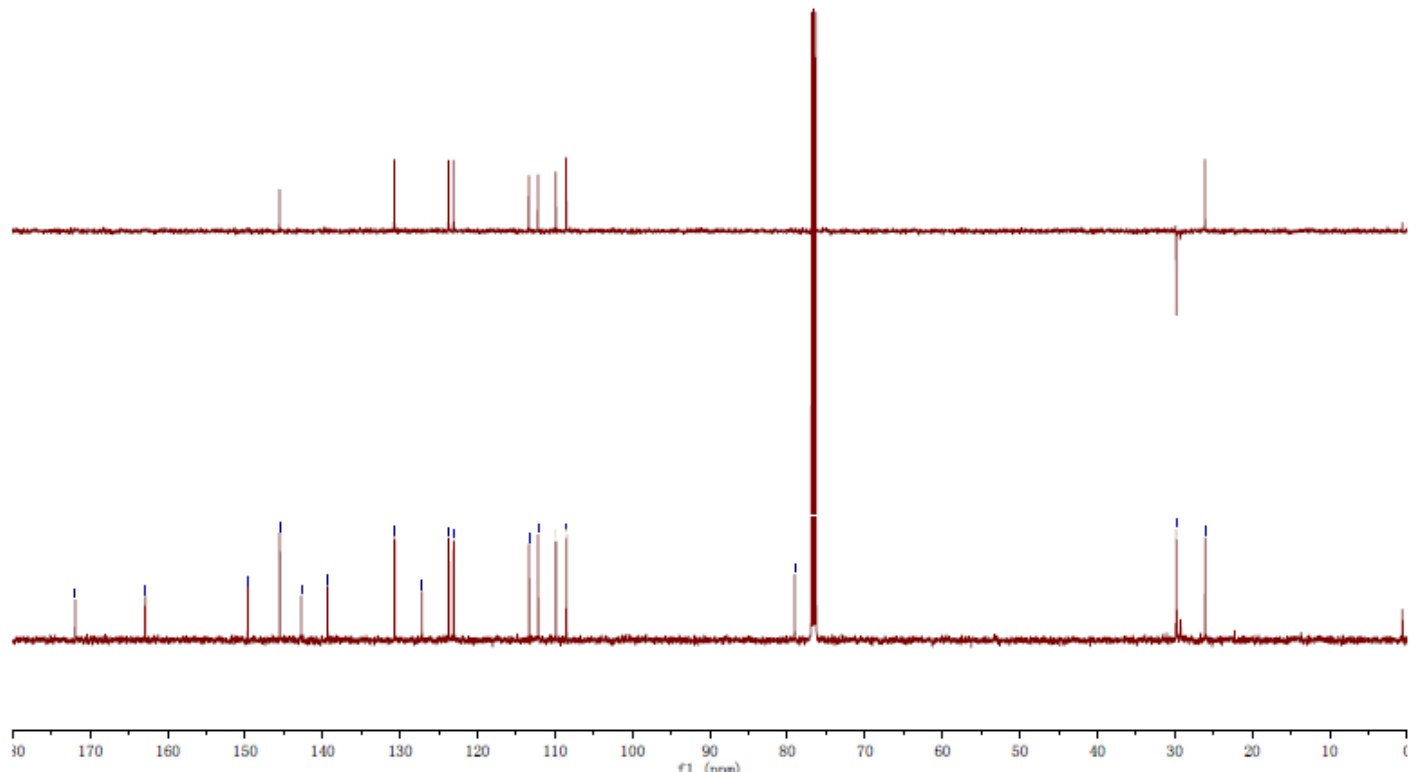
(R)-4'-cyclohexyl-1-methylspiro[indoline-3,2'-pyran]-2,6'(3'H)-dione (3h)

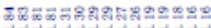

4
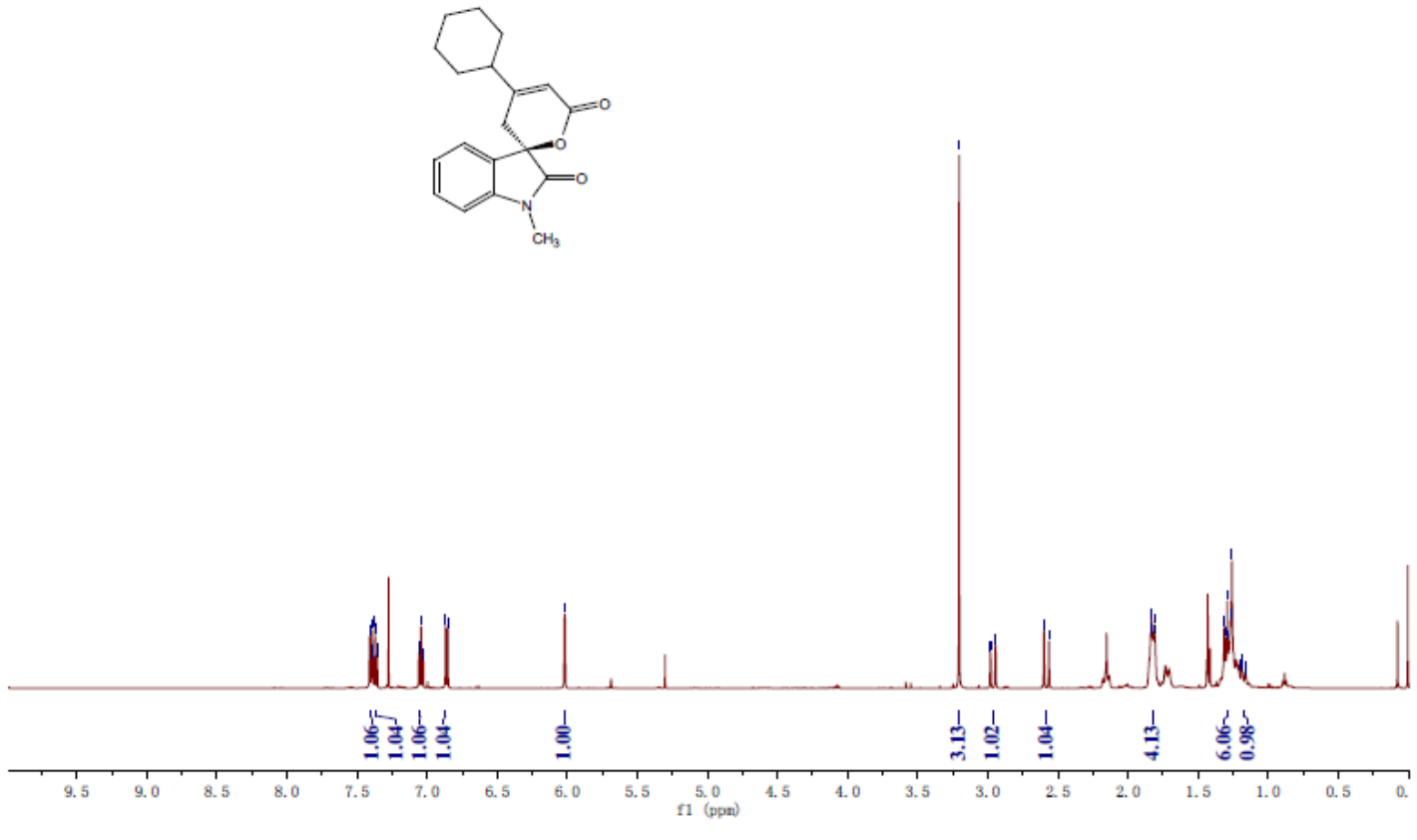

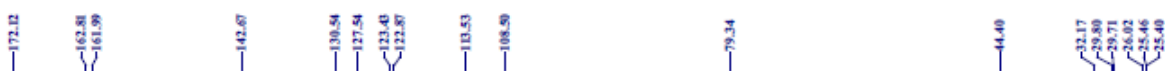
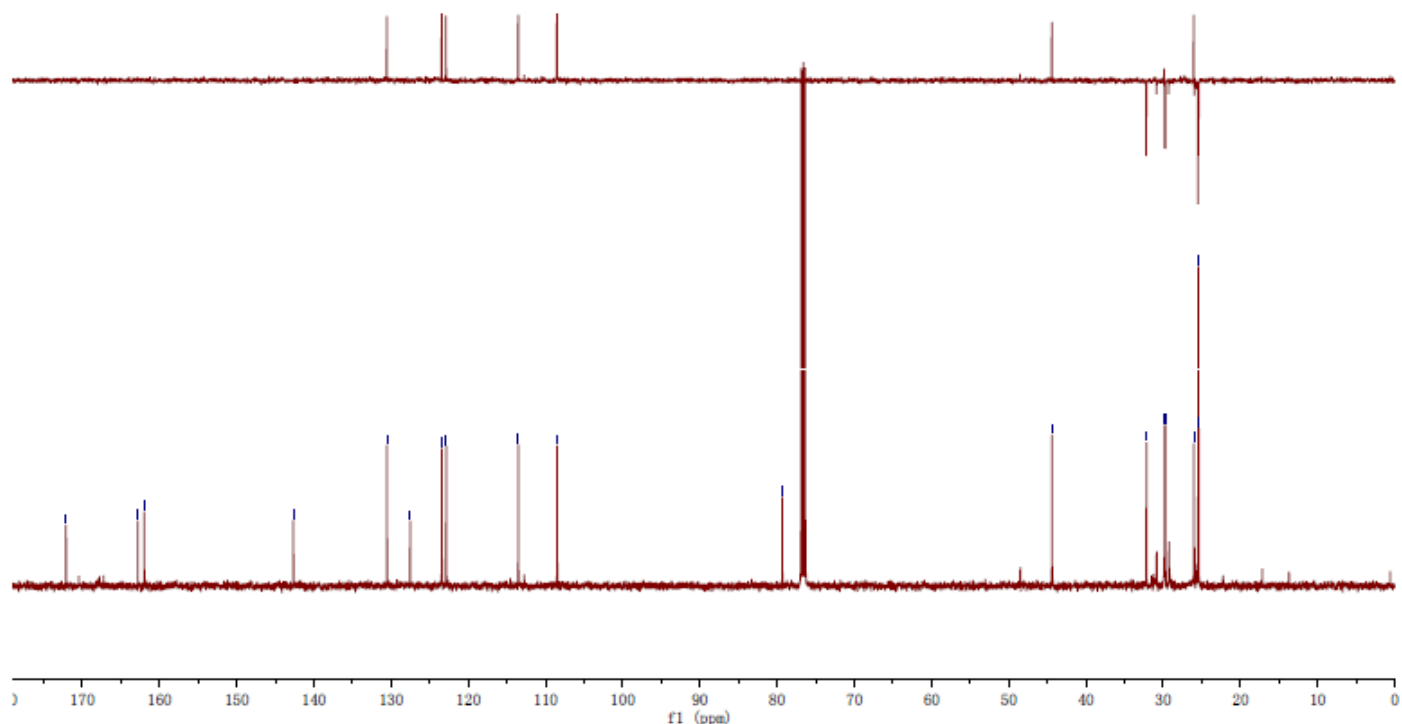
(R)-1,4'-dimethylspiro[indoline-3,2'-pyran]-2,6'(3'H)-dione (3i)

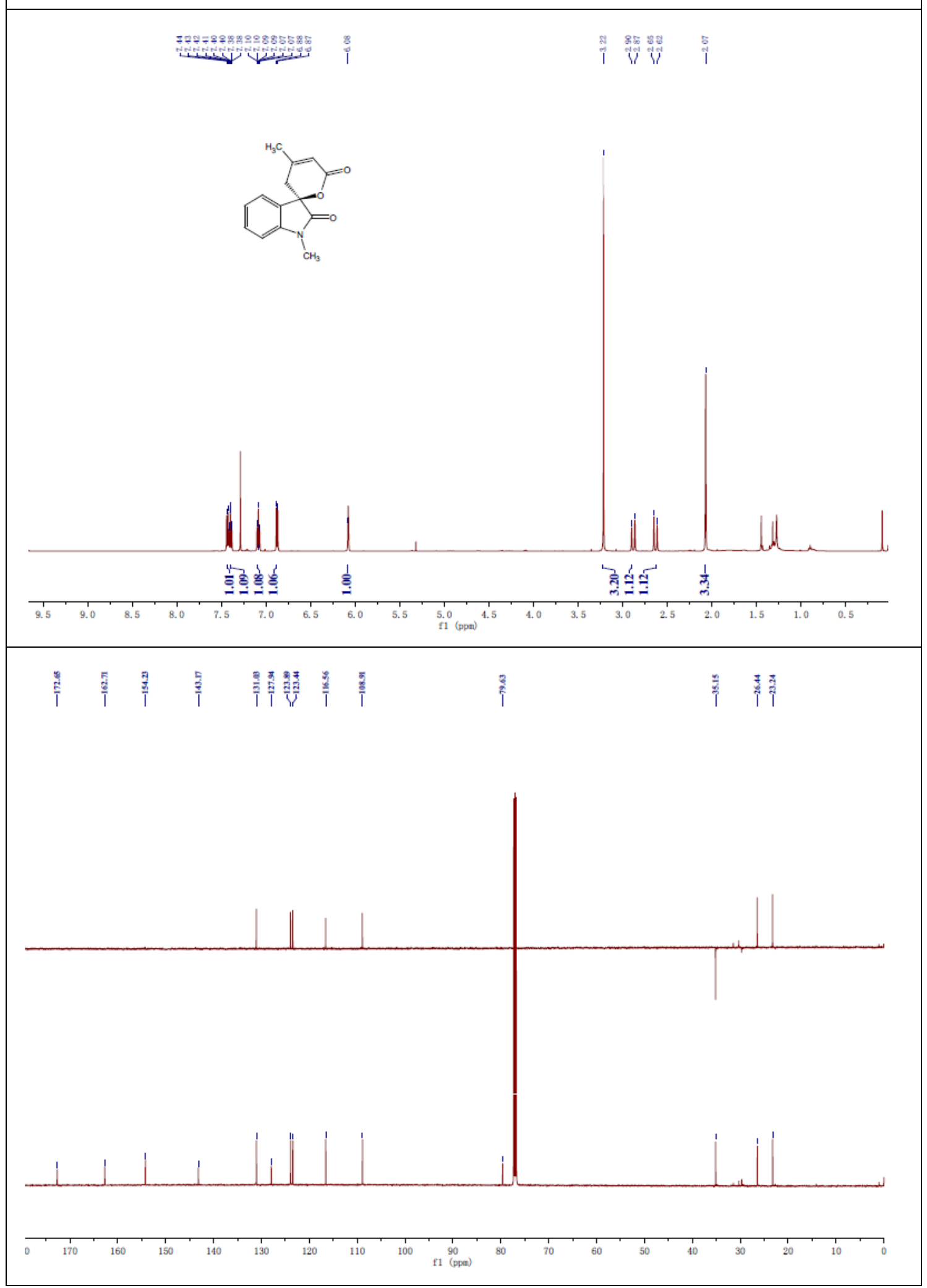




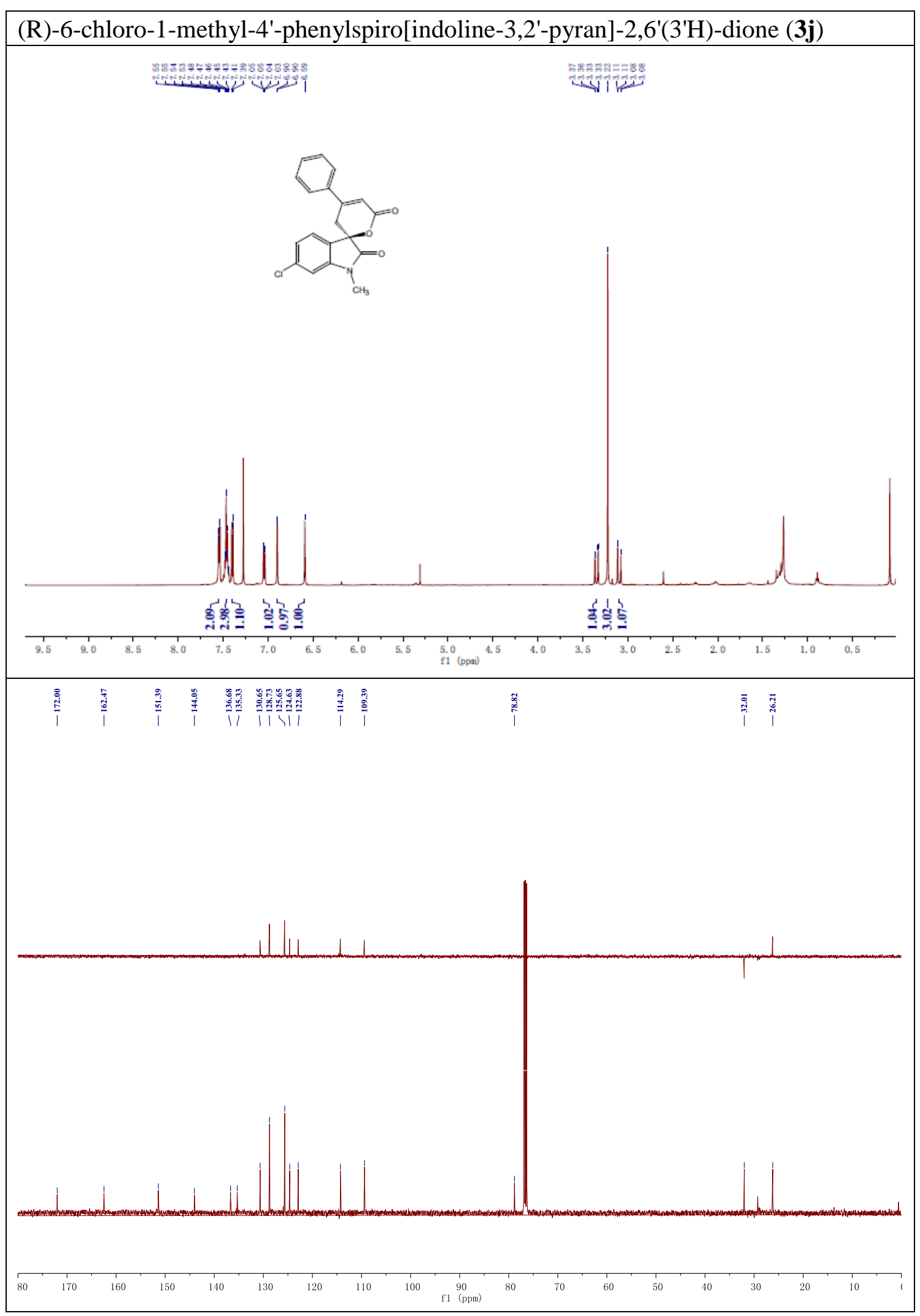




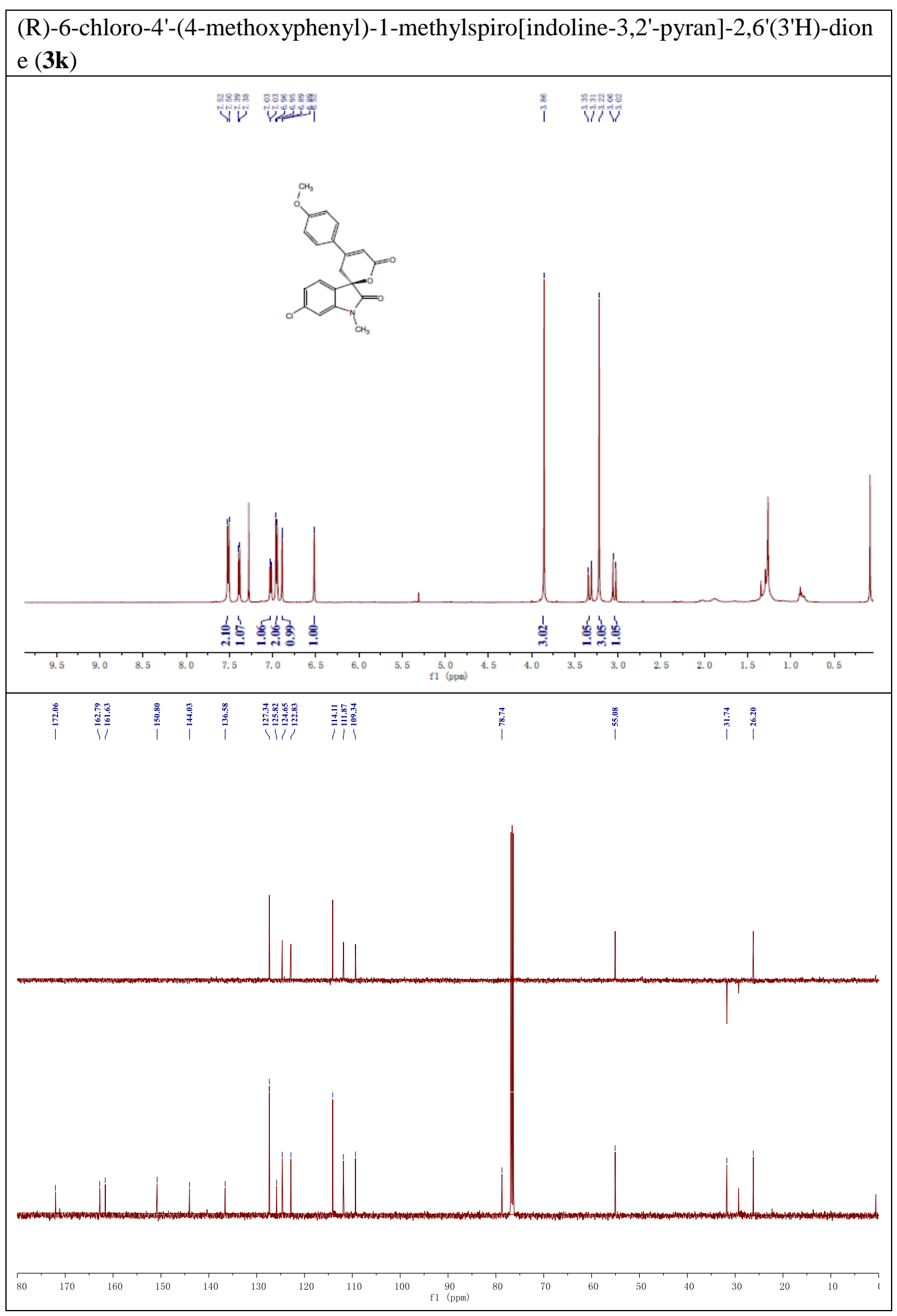




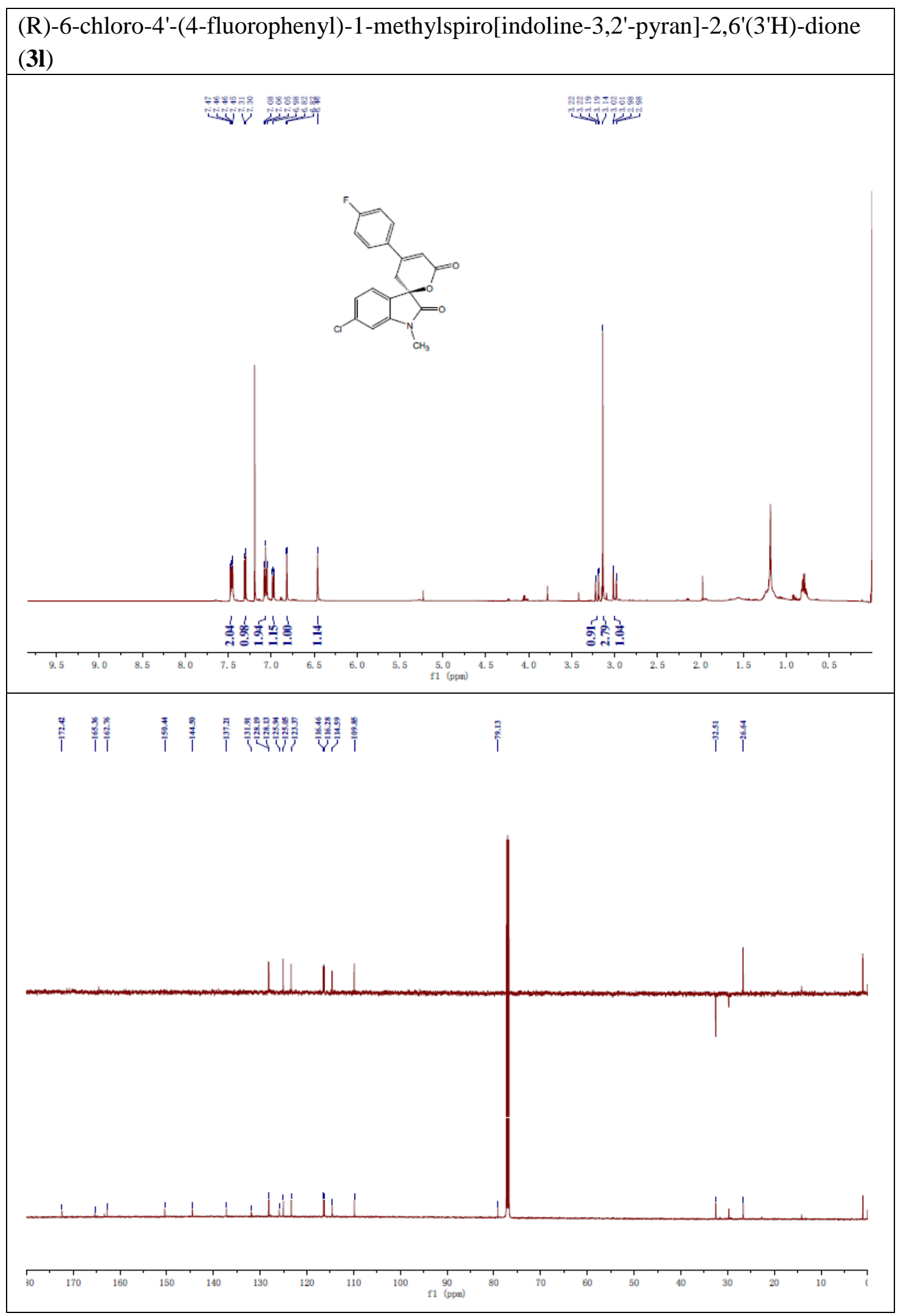




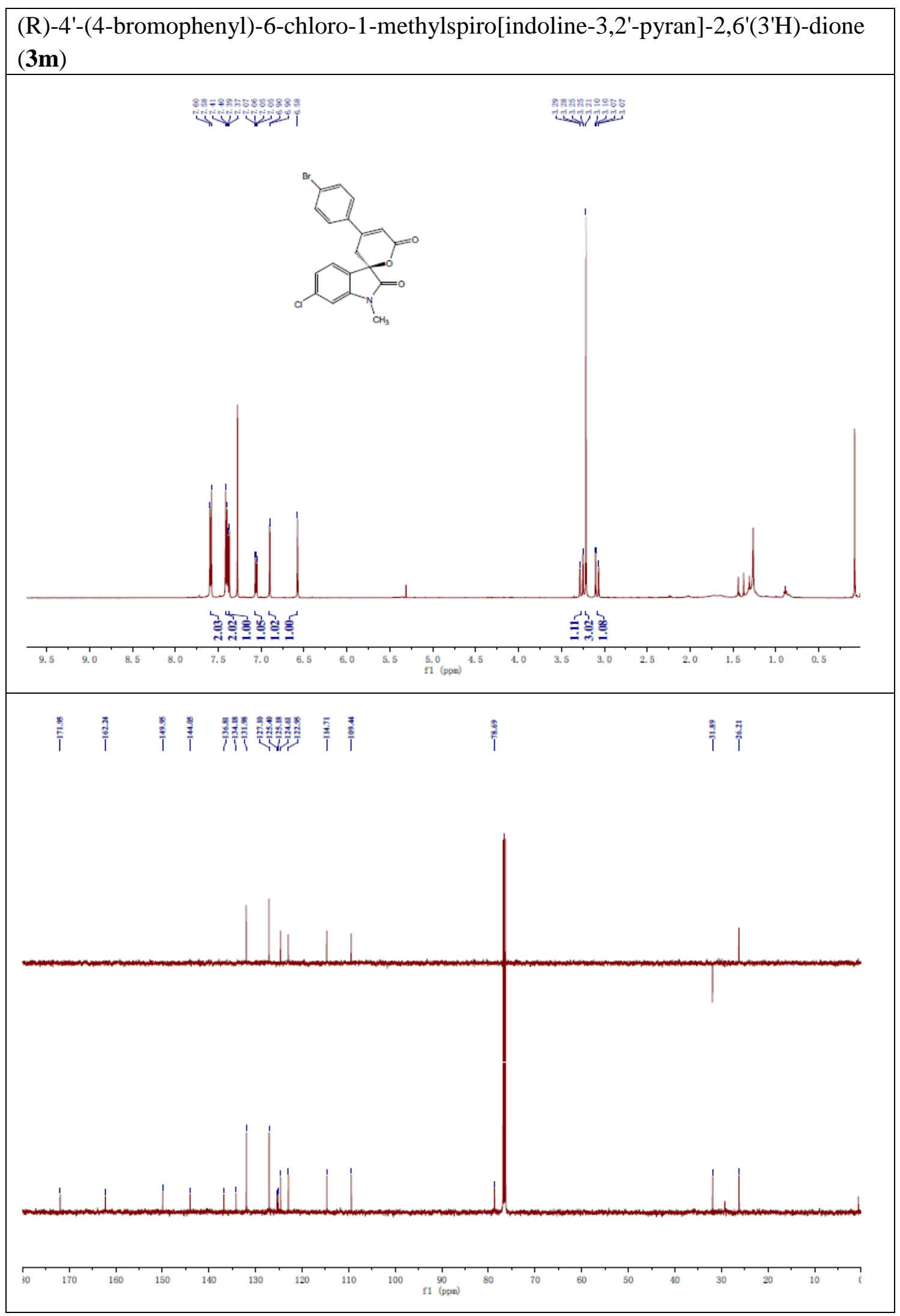


(R)-6-chloro-1,4'-dimethylspiro[indoline-3,2'-pyran]-2,6'(3'H)-dione (3n)

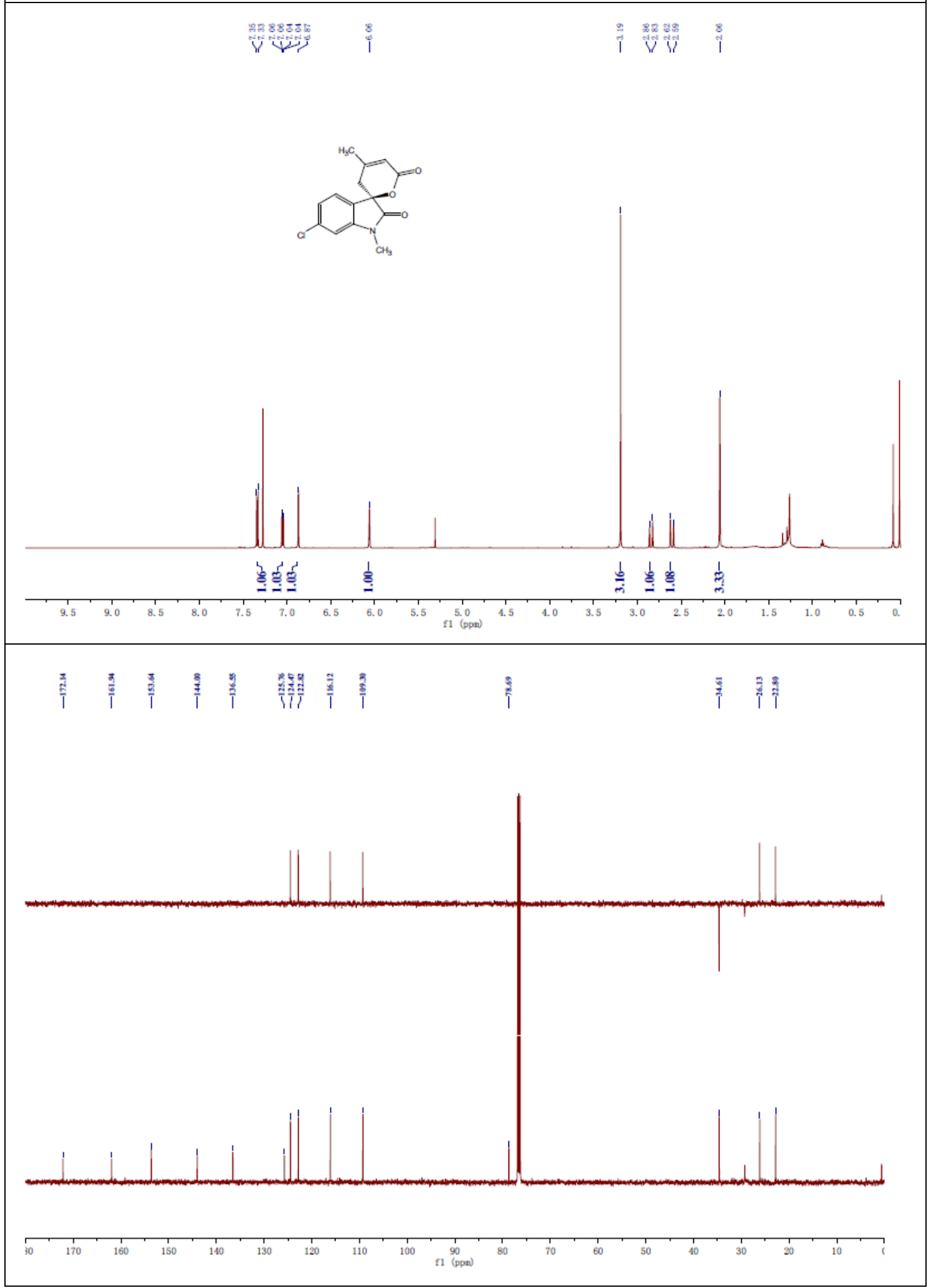


(R)-6-bromo-4'-(4-bromophenyl)-1-methylspiro[indoline-3,2'-pyran]-2,6'(3'H)-dione

(3o)

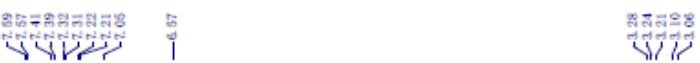
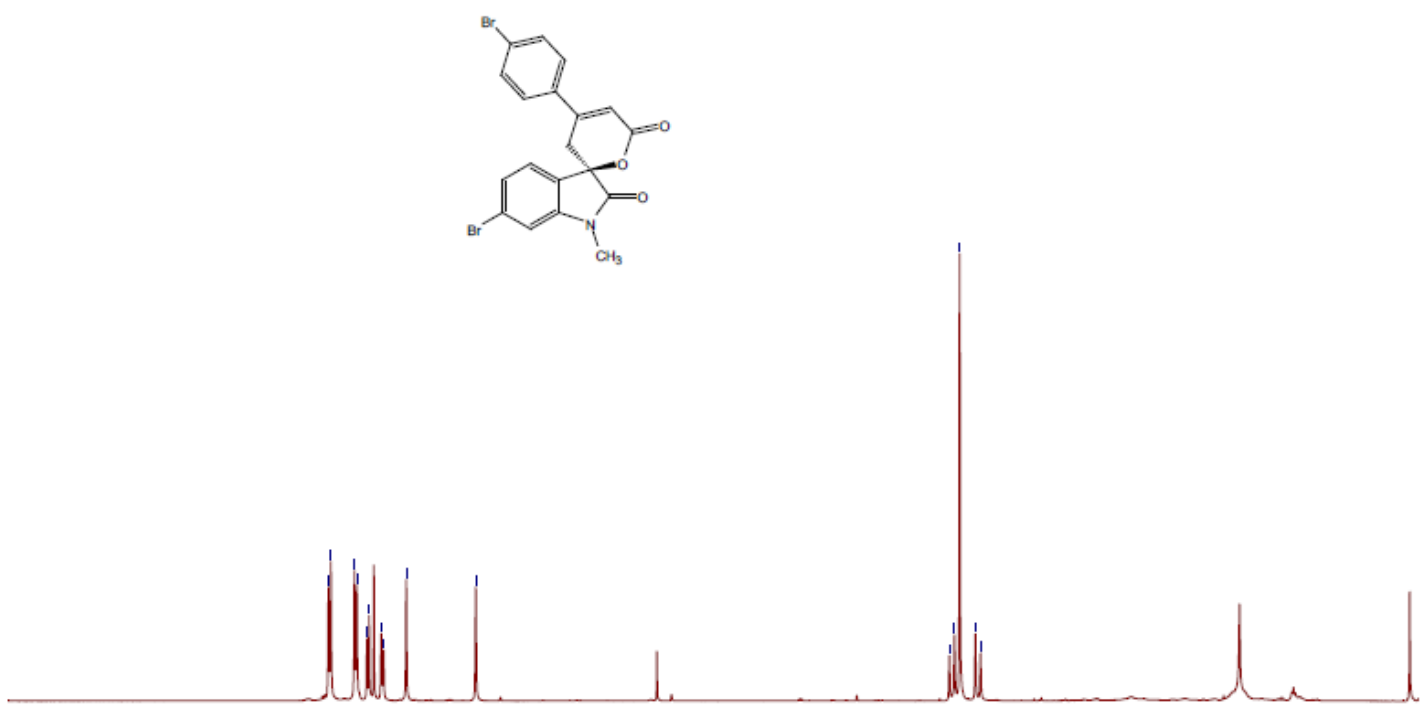

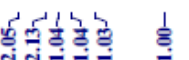

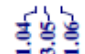

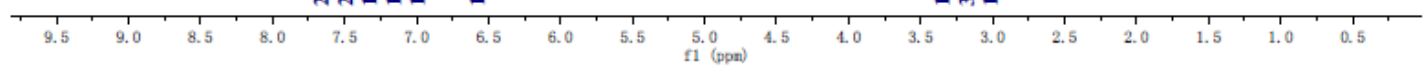

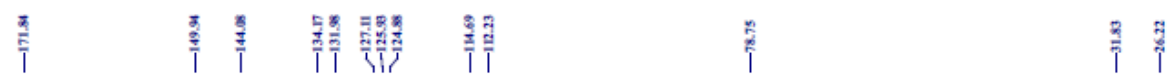

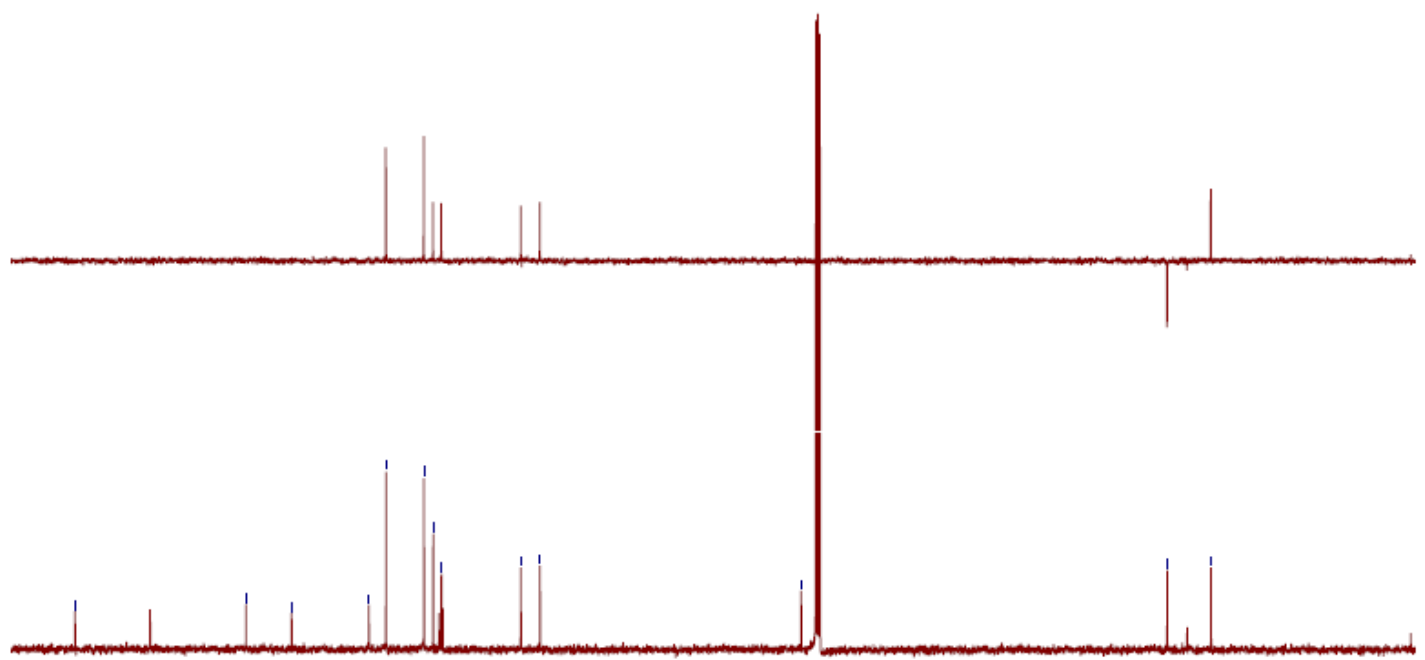

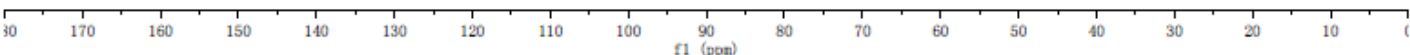


(R)-6-bromo-4'-(4-methoxyphenyl)-1-methylspiro[indoline-3,2'-pyran]-2,6'(3'H)-dion e $(3 \mathbf{p})$

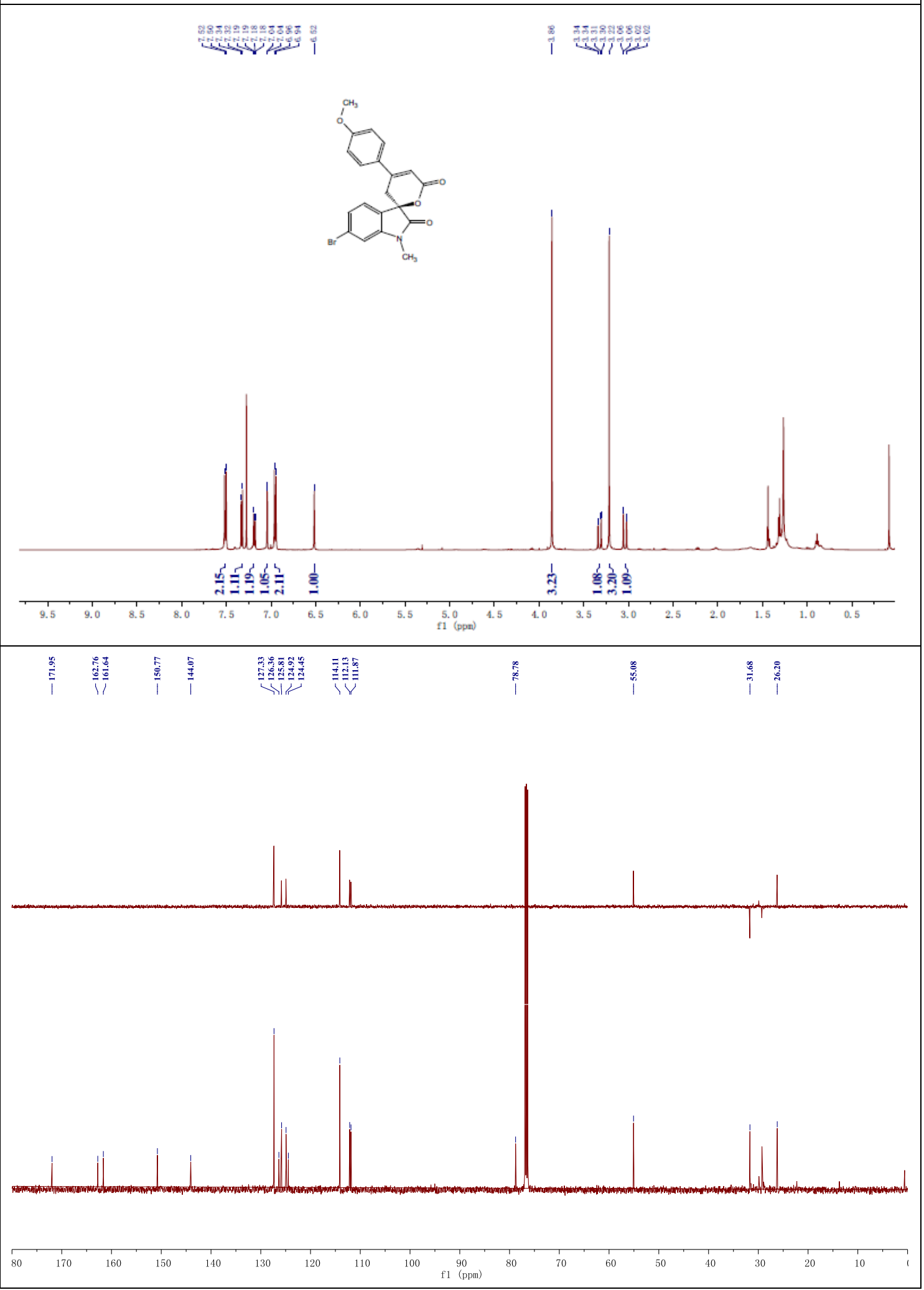




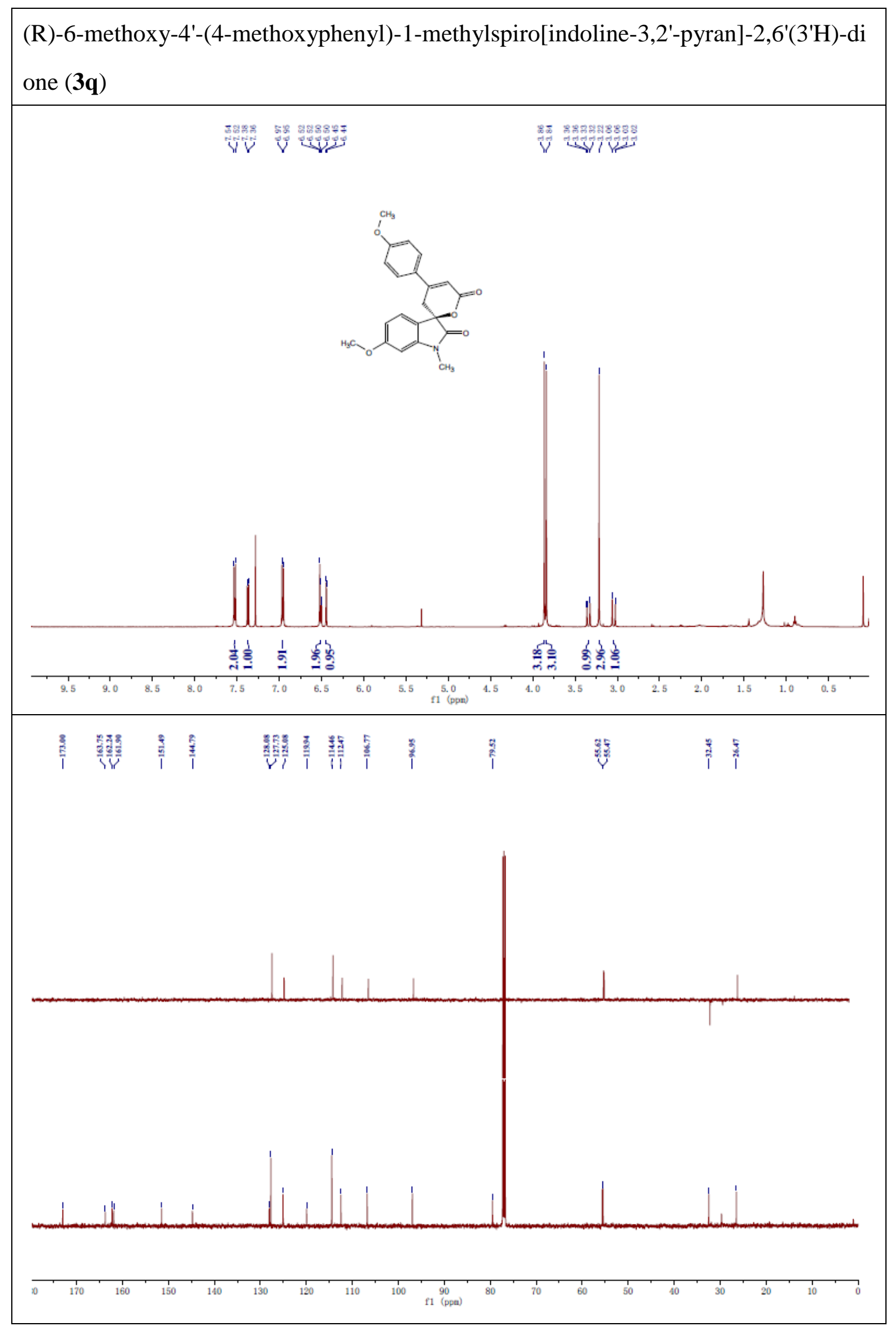

35 
(R)-5-fluoro-4'-(4-methoxyphenyl)-1-methylspiro[indoline-3,2'-pyran]-2,6'(3'H)-dion e (3r)

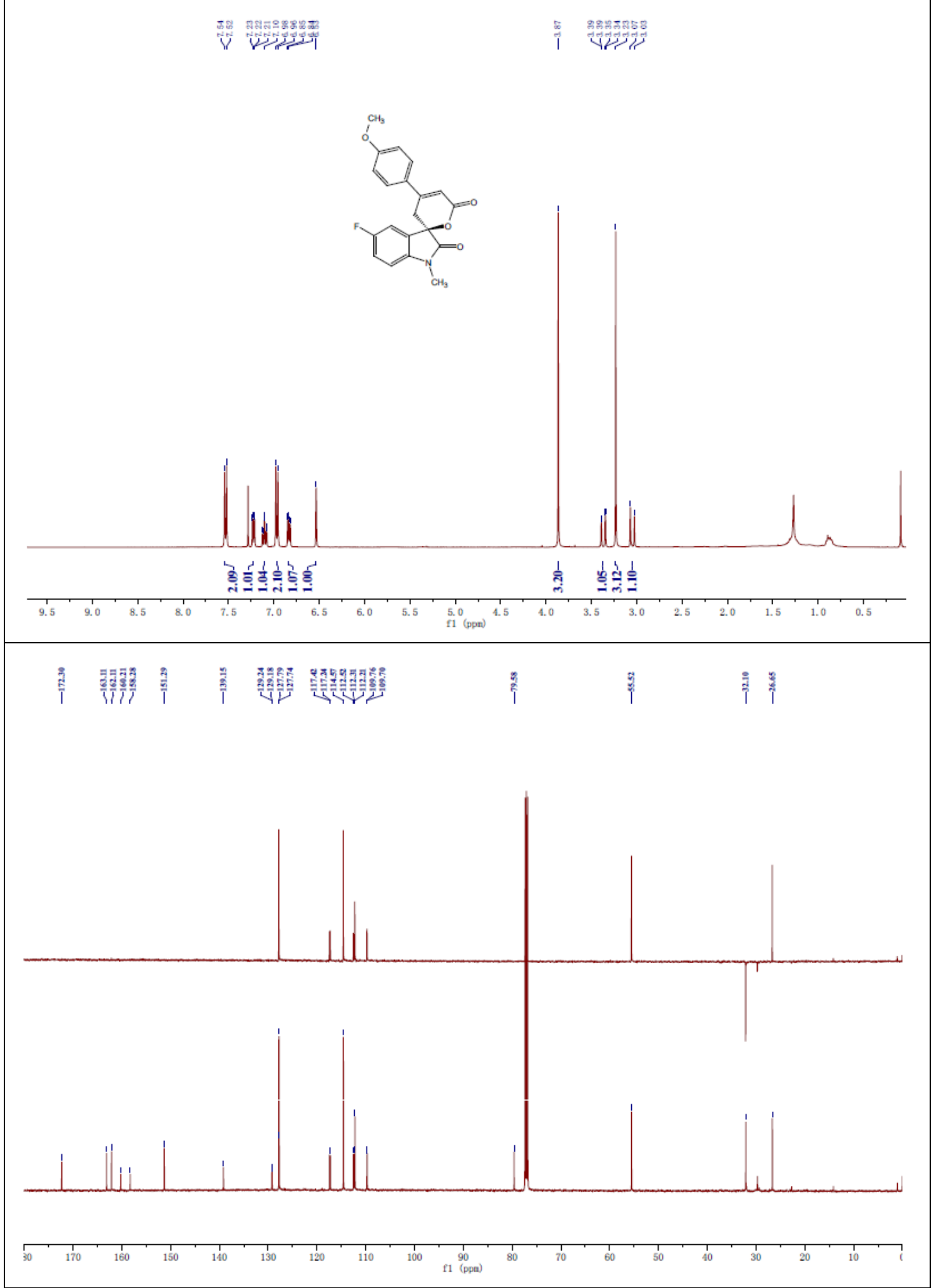


(R)-5-chloro-4'-(4-methoxyphenyl)-1-methylspiro[indoline-3,2'-pyran]-2,6'(3'H)-dion e (3s)

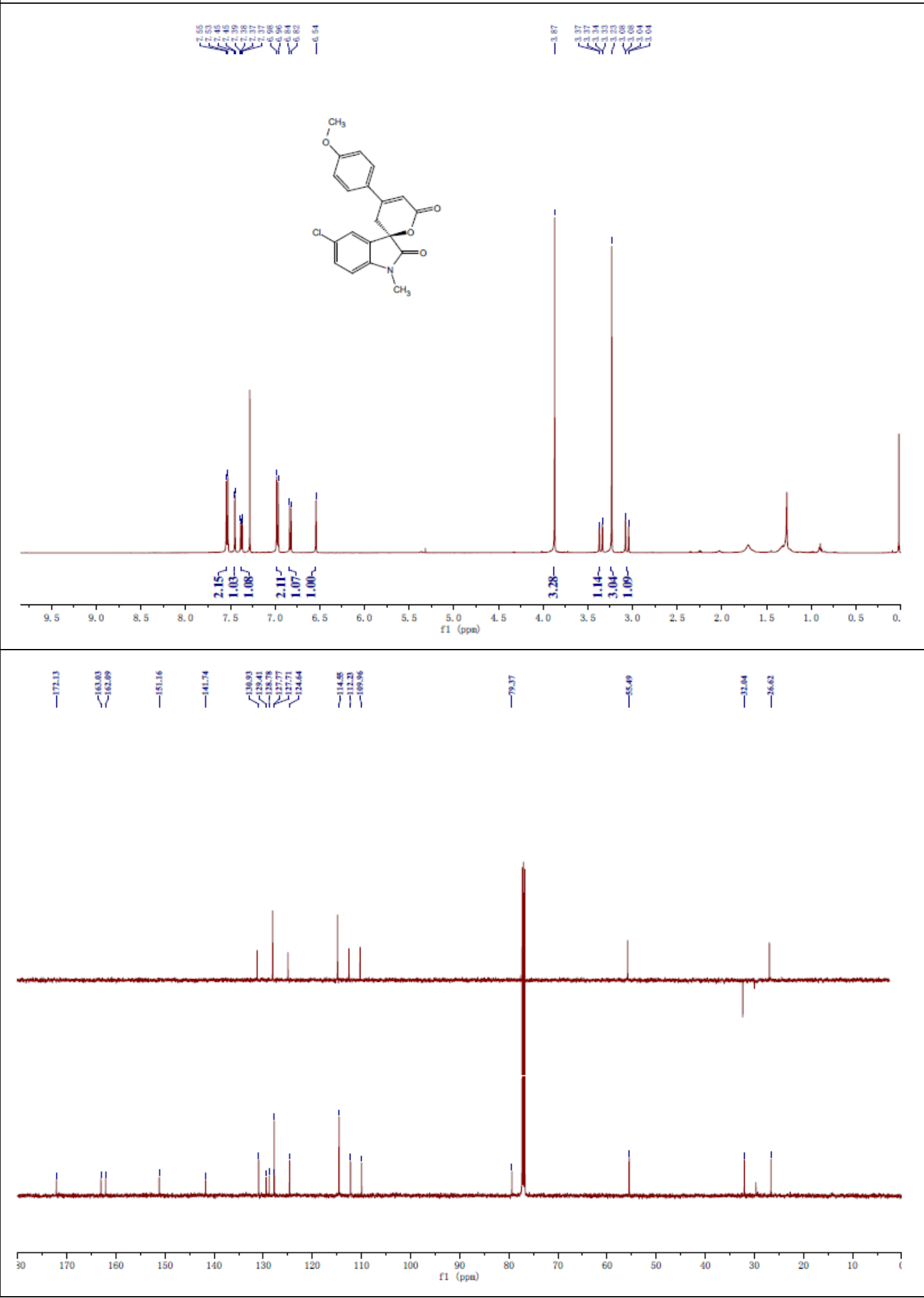


(R)-4-chloro-4'-(4-methoxyphenyl)-1-methylspiro[indoline-3,2'-pyran]-2,6'(3'H)-dion e (3t)

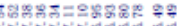

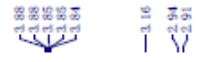
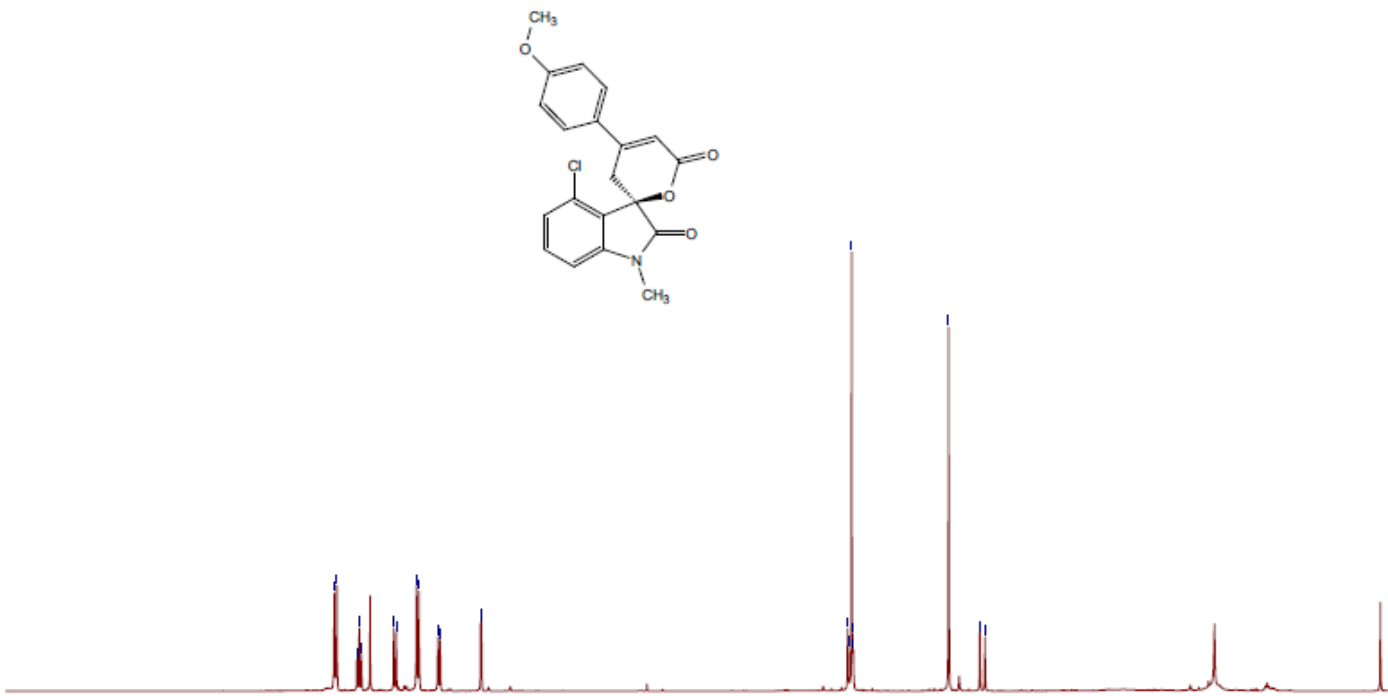

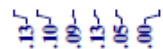
क्ष.

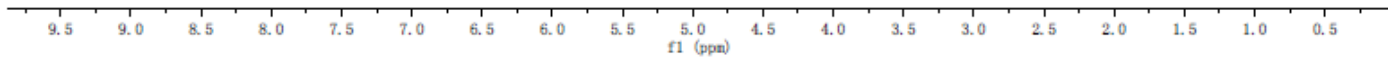

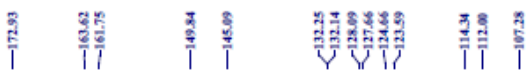

章

ํํำ

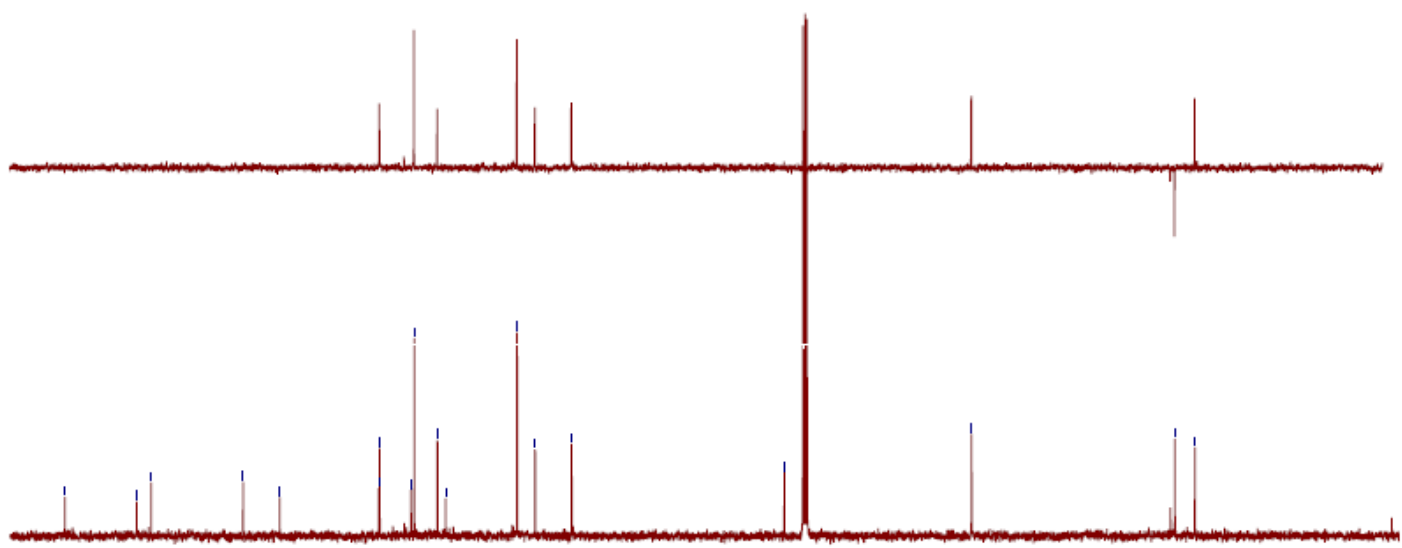

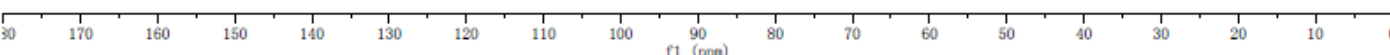




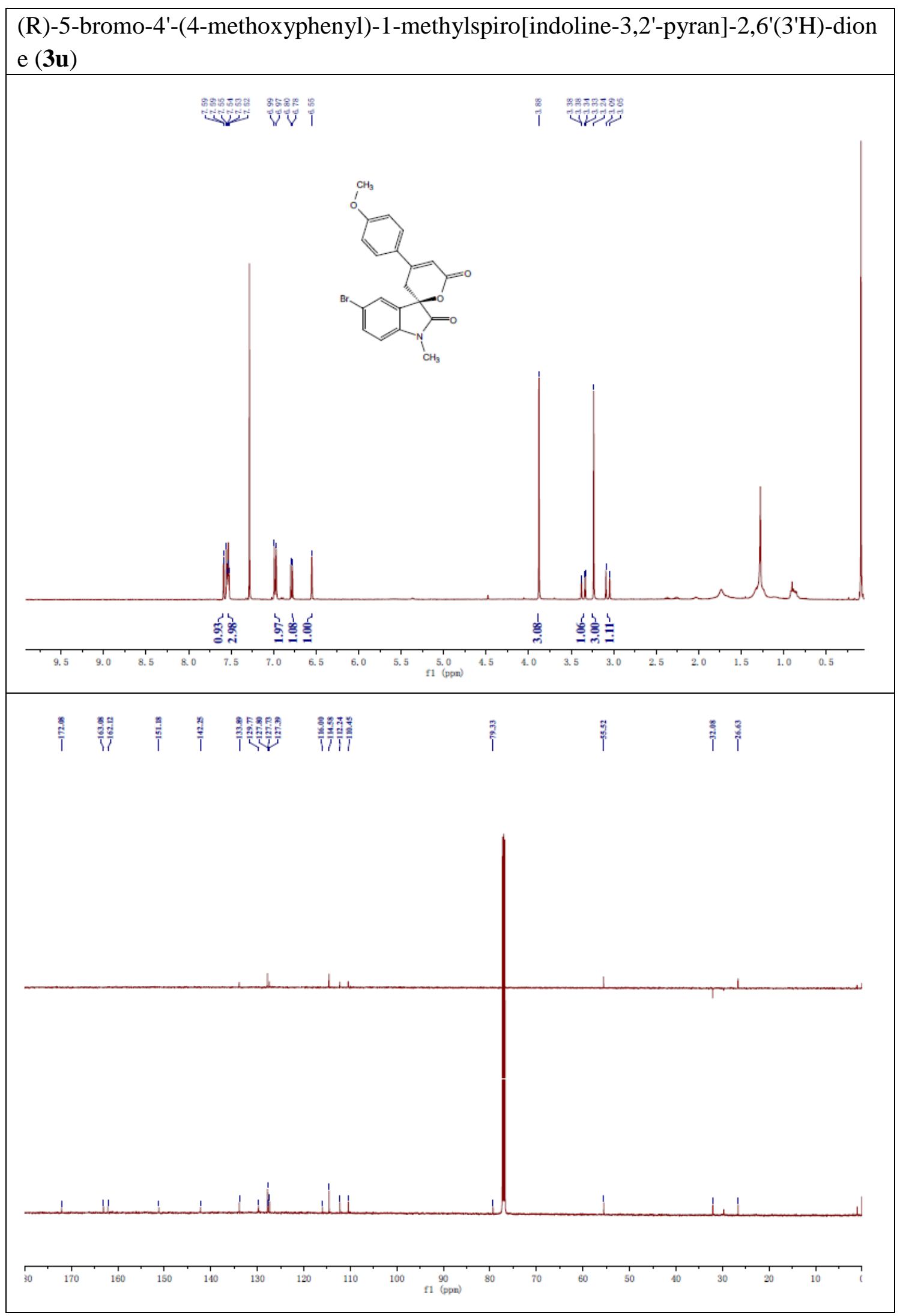


(R)-5-methoxy-4'-(4-methoxyphenyl)-1-methylspiro[indoline-3,2'-pyran]-2,6'(3'H)-di one (3v)

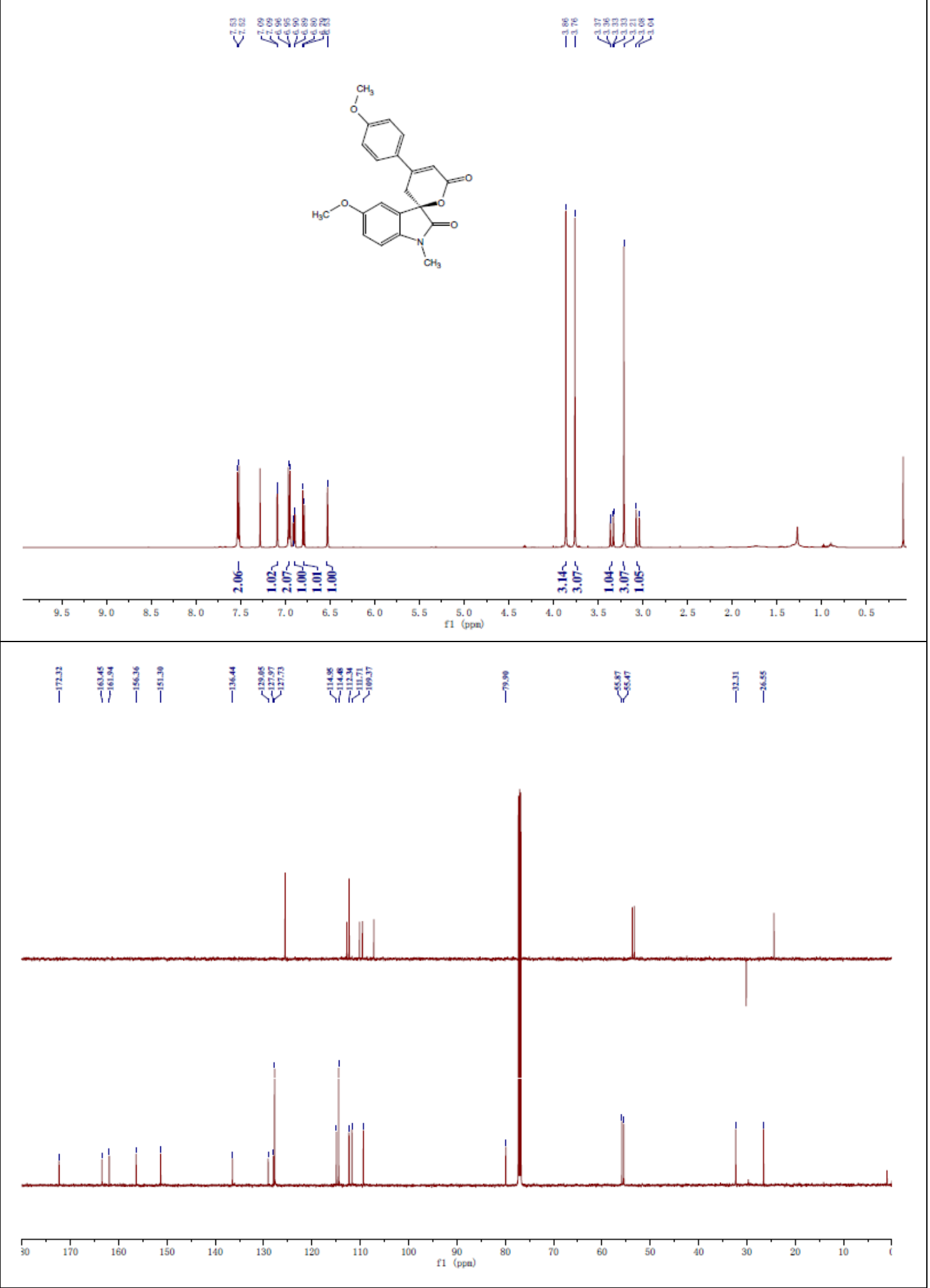




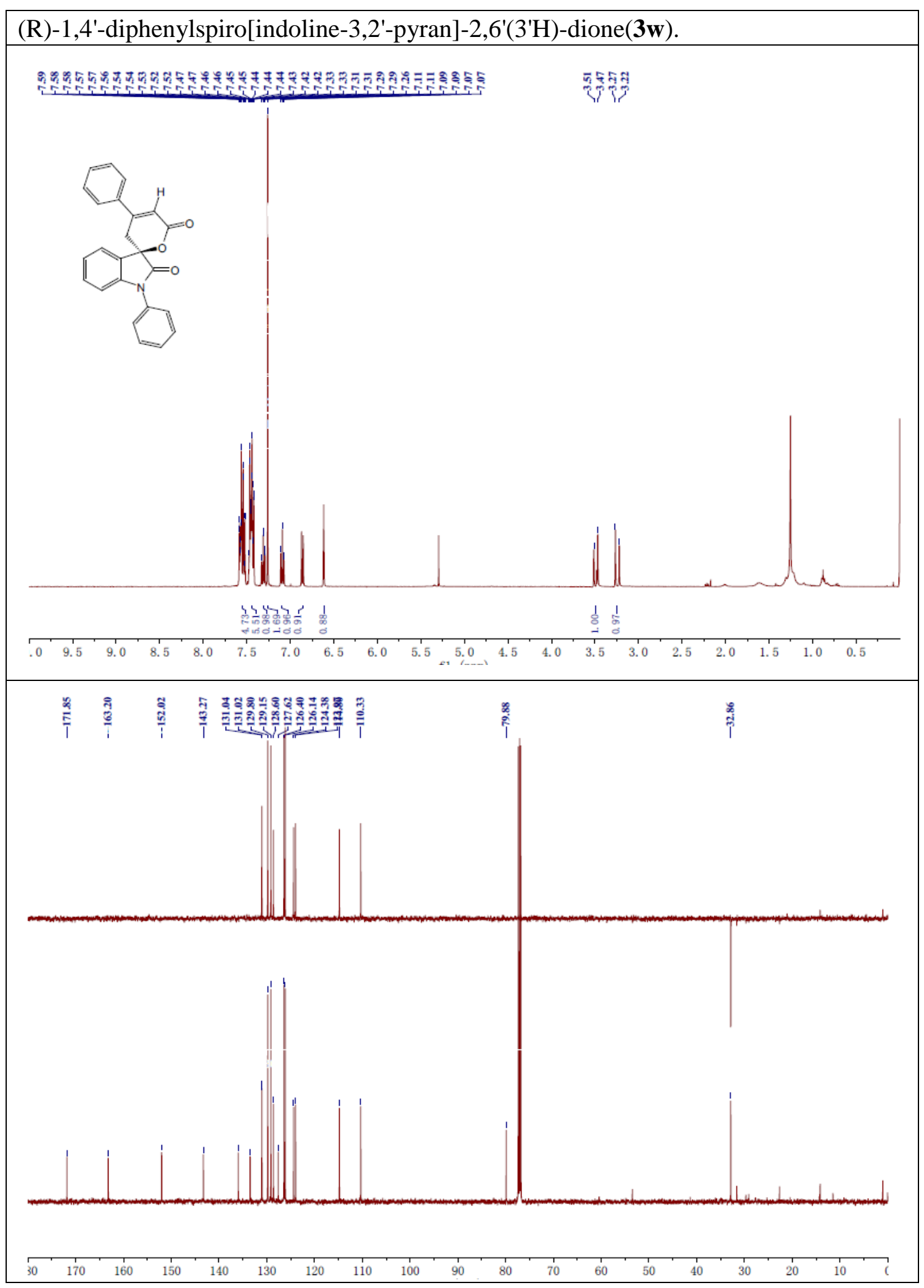




\section{Part IV HPLC Spectra}

(R)-1-methyl-4'-phenylspiro[indoline-3,2'-pyran]-2,6'(3'H)-dione(3a)

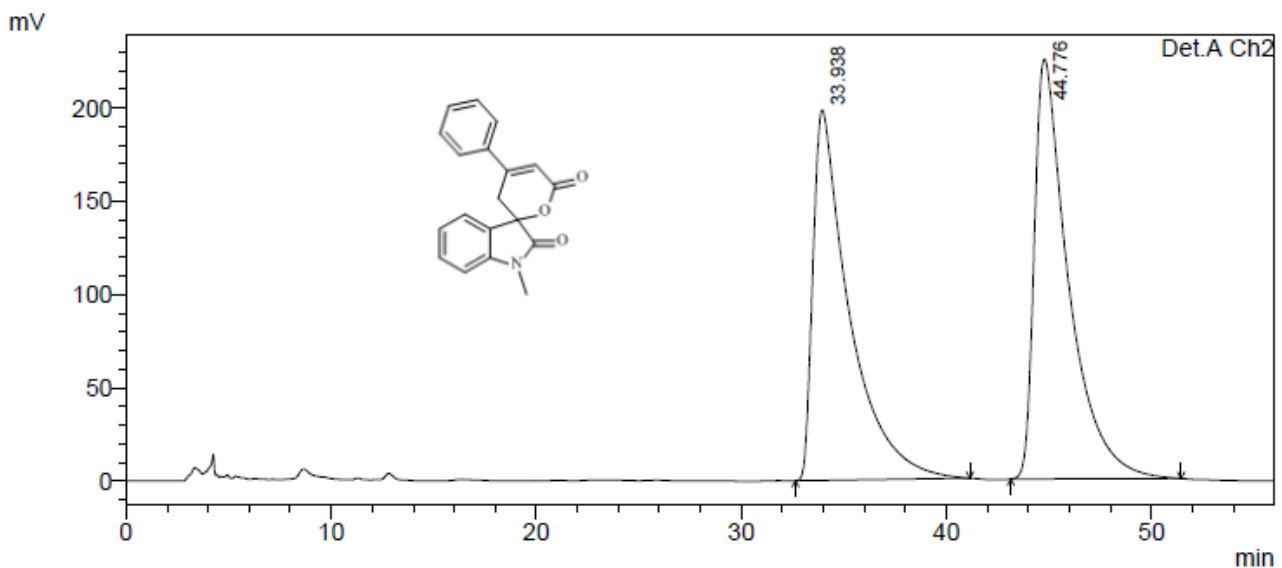

Detector A Ch2 254nm

\begin{tabular}{|r|r|r|r|r|r|}
\hline Peak\# & Ret. Time & \multicolumn{1}{c|}{ Area } & \multicolumn{1}{c|}{ Height } & \multicolumn{1}{c|}{ Area \% } & \multicolumn{1}{c|}{ Height \% } \\
\hline 1 & 33.938 & 24437308 & 198662 & 48.331 & 46.864 \\
\hline 2 & 44.776 & 26124829 & 225249 & 51.669 & 53.136 \\
\hline Total & & 50562137 & 423911 & 100.000 & 100.000 \\
\hline
\end{tabular}

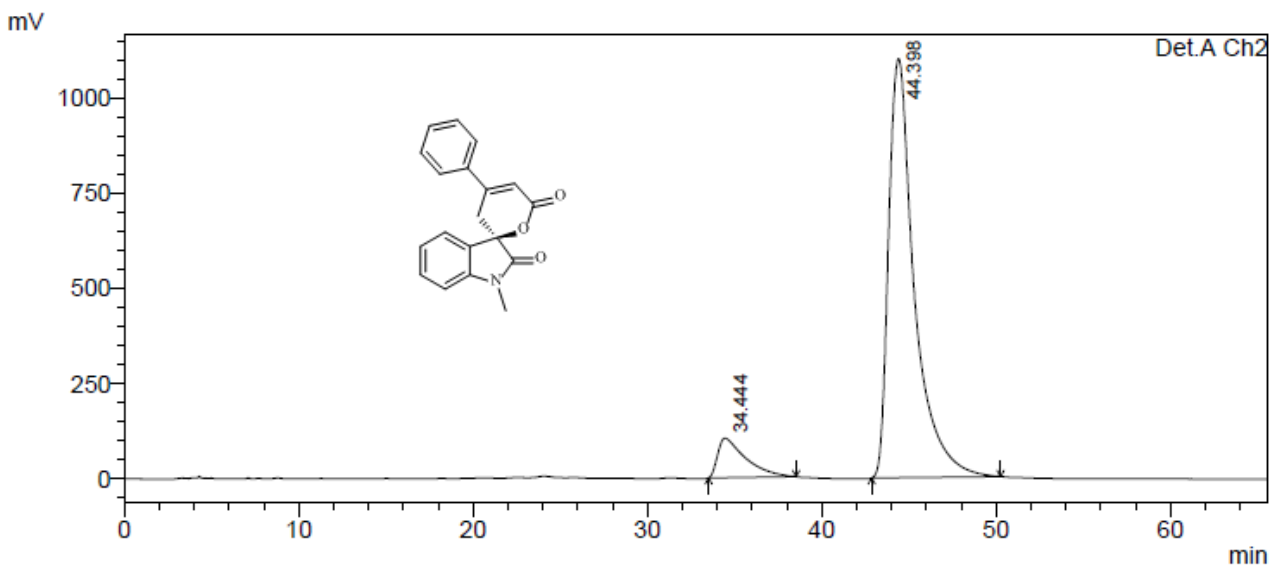

Detector A Ch2 254nm

\begin{tabular}{|r|r|r|r|r|r|}
\hline \multicolumn{1}{|c|}{ Peak\# } & \multicolumn{1}{c|}{ Ret. Time } & \multicolumn{1}{c|}{ Area } & \multicolumn{1}{c|}{ Height } & \multicolumn{1}{c|}{ Area \% } & \multicolumn{1}{c|}{ Height \% } \\
\hline 1 & 34.444 & 11545531 & 103559 & 9.270 & 8.605 \\
\hline 2 & 44.398 & 112997767 & 1099930 & 90.730 & 91.395 \\
\hline Total & & 124543298 & 1203488 & 100.000 & 100.000 \\
\hline
\end{tabular}


(R)-4'-(4-methoxyphenyl)-1-methylspiro[indoline-3,2'-pyran]-2,6'(3'H)-dione(3b)

$\mathrm{mV}$

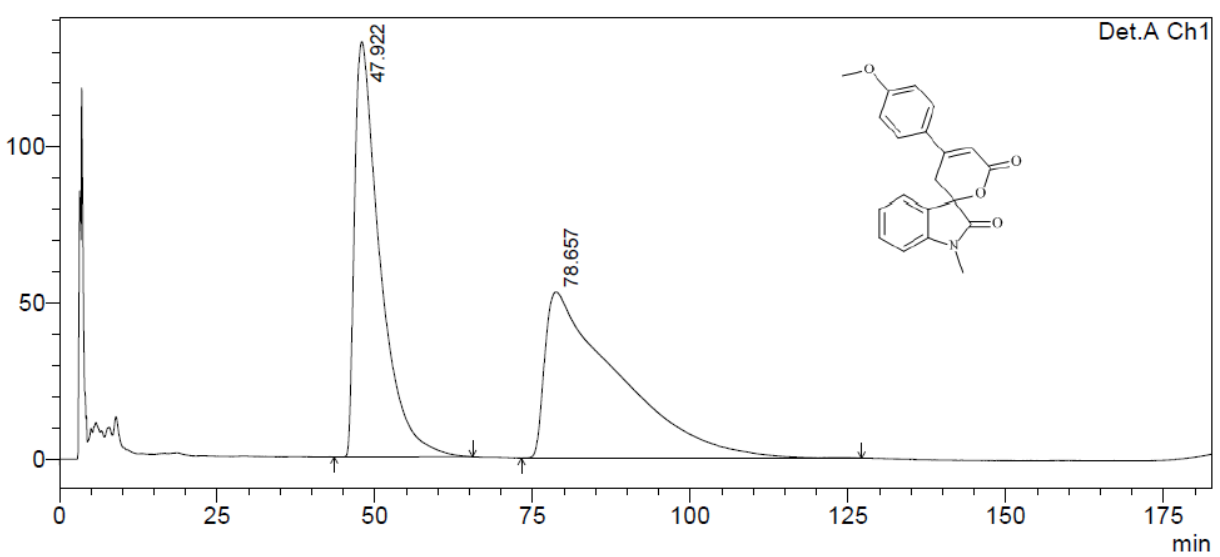

\begin{tabular}{|r|r|r|r|r|r|}
\hline \multicolumn{1}{|c|}{ Peak\# } & Ret. Time & \multicolumn{1}{c|}{ Area } & Height & \multicolumn{1}{c|}{ Area \% } & \multicolumn{1}{c|}{ Height \% } \\
\hline 1 & 47.922 & 37355501 & 132585 & 46.457 & 71.412 \\
\hline 2 & 78.657 & 43052725 & 53078 & 53.543 & 28.588 \\
\hline Total & & 80408226 & 185662 & 100.000 & 100.000 \\
\hline
\end{tabular}

$\mathrm{mV}$

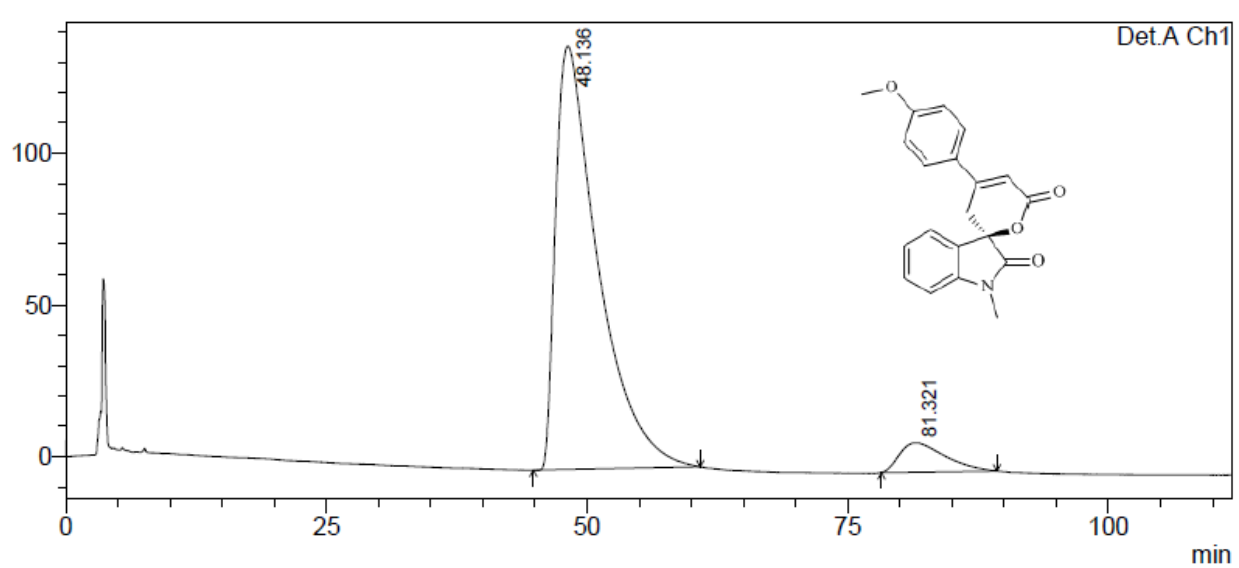

\begin{tabular}{|r|r|r|r|r|r|}
\hline \multicolumn{1}{|c|}{ Peak\# } & Ret. Time & \multicolumn{1}{c|}{ Area } & \multicolumn{1}{c|}{ Height } & \multicolumn{1}{c|}{ Area \% } & \multicolumn{1}{c|}{ Height \% } \\
\hline 1 & 48.136 & 39459754 & 139518 & 93.087 & 93.499 \\
\hline 2 & 81.321 & 2930250 & 9701 & 6.913 & 6.501 \\
\hline Total & & 42390004 & 149219 & 100.000 & 100.000 \\
\hline
\end{tabular}


(R)-1-methyl-4'-(4-nitrophenyl)spiro[indoline-3,2'-pyran]-2,6'(3'H)-dione(3c)

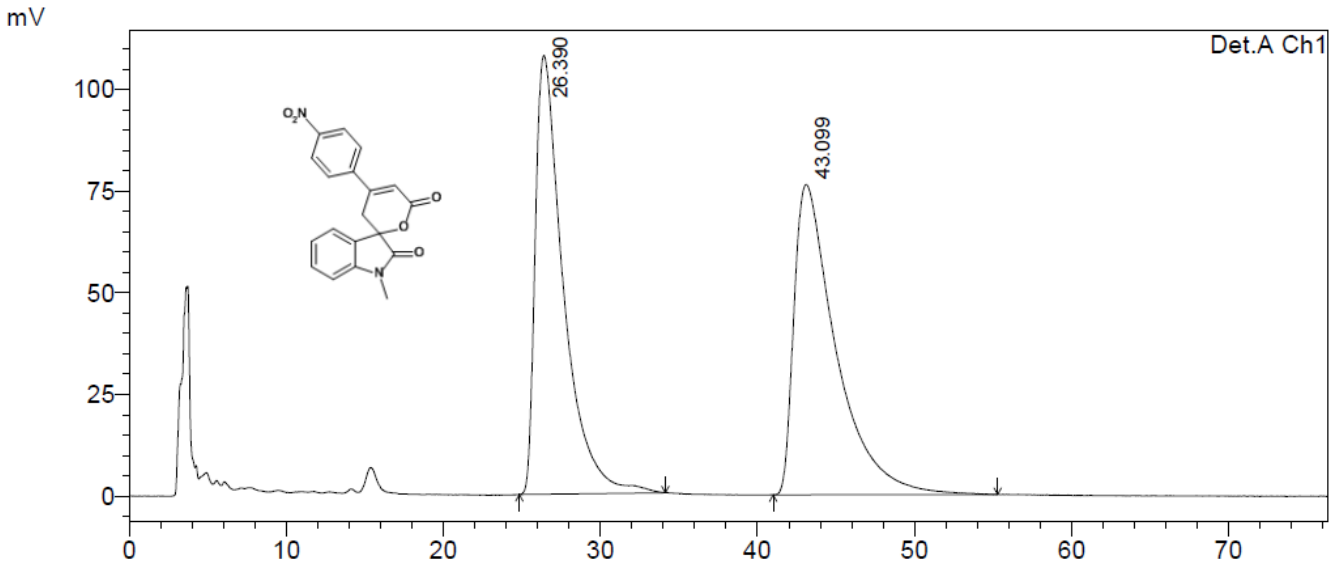

\begin{tabular}{|r|r|r|r|r|r|}
\hline \multicolumn{1}{|c|}{ Peak\# } & Ret. Time & \multicolumn{1}{|c|}{ Area } & Height & Area \% & Height \% \\
\hline 1 & 26.390 & 13460969 & 107886 & 48.773 & 58.598 \\
\hline 2 & 43.099 & 14138041 & 76226 & 51.227 & 41.402 \\
\hline Total & & 27599009 & 184112 & 100.000 & 100.000 \\
\hline
\end{tabular}

$\mathrm{mV}$

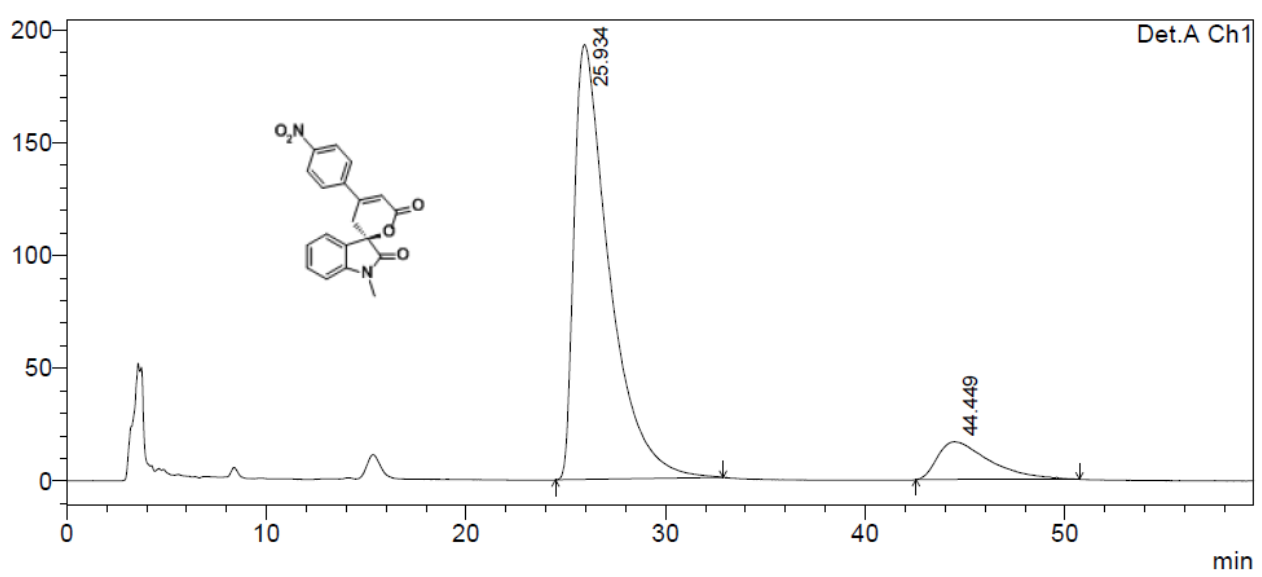

\begin{tabular}{|r|r|r|r|r|r|}
\hline \multicolumn{1}{|c|}{ Peak\# } & \multicolumn{1}{|c|}{ Ret. Time } & \multicolumn{1}{c|}{ Area } & \multicolumn{1}{c|}{ Height } & Area $\%$ & \multicolumn{1}{|c|}{ Height $\%$} \\
\hline 1 & 25.934 & 23626476 & 192868 & 88.625 & 92.004 \\
\hline 2 & 44.449 & 3032379 & 16762 & 11.375 & 7.996 \\
\hline Total & & 26658854 & 209630 & 100.000 & 100.000 \\
\hline
\end{tabular}


(R)-4'-(4-fluorophenyl)-1-methylspiro[indoline-3,2'-pyran]-2,6'(3'H)-dione(3d)

$\mathrm{mV}$

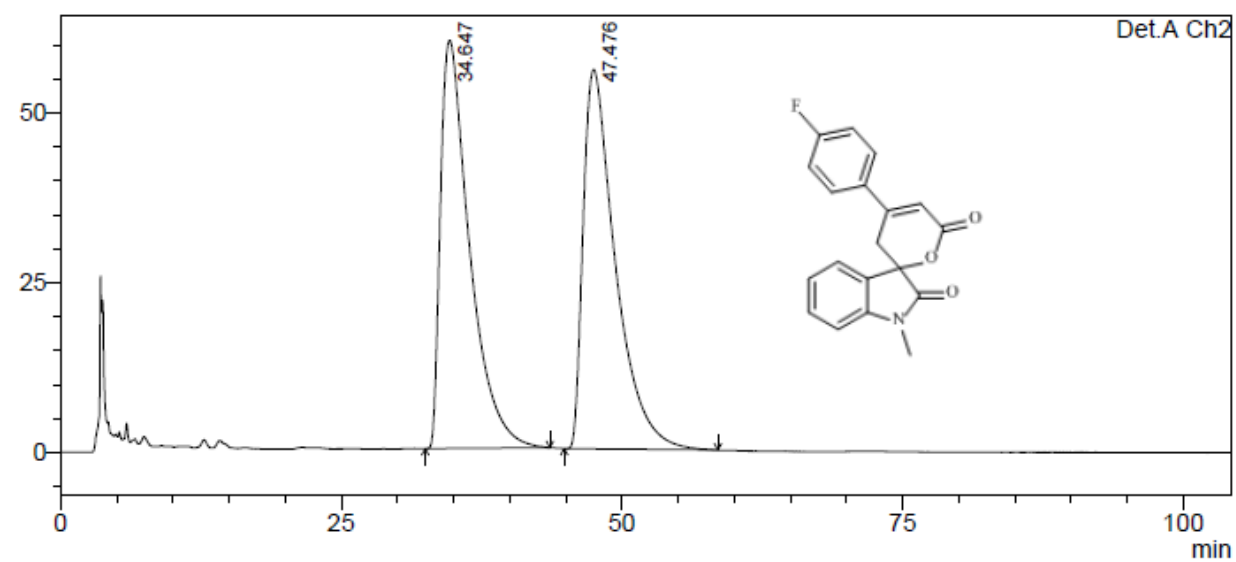

Detector A Ch1 190nm

\begin{tabular}{|r|r|r|r|r|r|}
\hline \multicolumn{1}{|c|}{ Peak\# } & Ret. Time & \multicolumn{1}{c|}{ Area } & Height & Area \% & \multicolumn{1}{c|}{ Height \% } \\
\hline 1 & 34.702 & 10715843 & 56484 & 50.718 & 51.594 \\
\hline 2 & 47.527 & 10412274 & 52994 & 49.282 & 48.406 \\
\hline Total & & 21128117 & 109478 & 100.000 & 100.000 \\
\hline
\end{tabular}

$\mathrm{mV}$

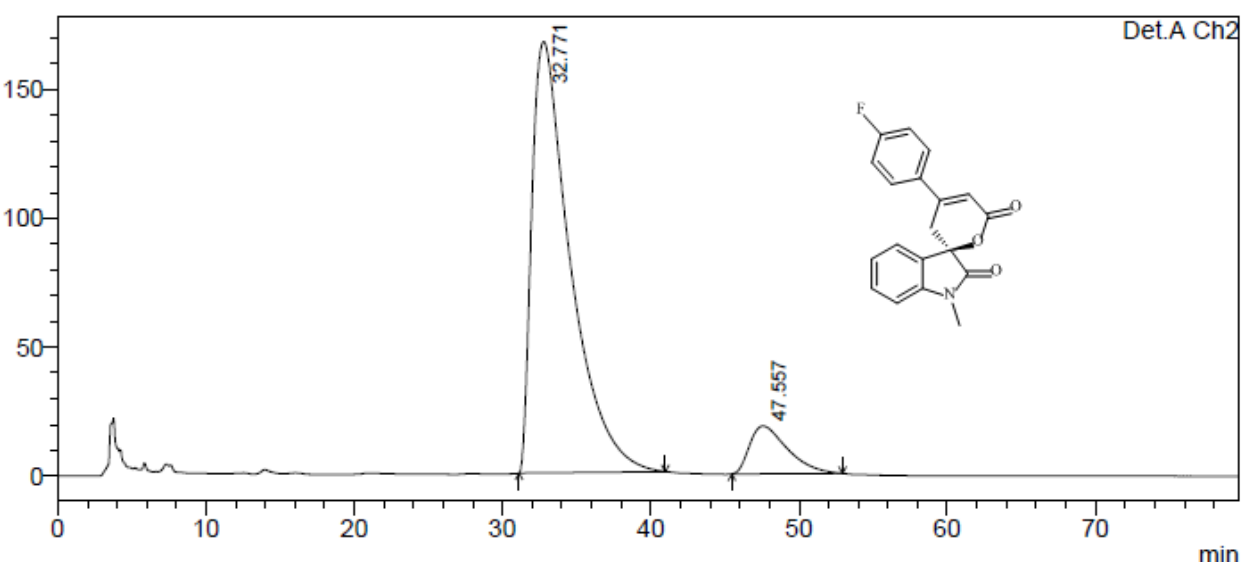

Detector A Ch1 190nm

\begin{tabular}{|r|r|r|r|r|r|}
\hline \multicolumn{1}{|c|}{ Peak\# } & Ret. Time & \multicolumn{1}{c|}{ Area } & \multicolumn{1}{c|}{ Height } & \multicolumn{1}{c|}{ Area \% } & \multicolumn{1}{c|}{ Height \% } \\
\hline 1 & 32.814 & 24191442 & 135902 & 87.339 & 88.303 \\
\hline 2 & 47.898 & 3506953 & 18003 & 12.661 & 11.697 \\
\hline Total & & 27698394 & 153905 & 100.000 & 100.000 \\
\hline
\end{tabular}


(R)-4'-(4-chlorophenyl)-1-methylspiro[indoline-3,2'-pyran]-2,6'(3'H)-dione(3e)

$\mathrm{mV}$

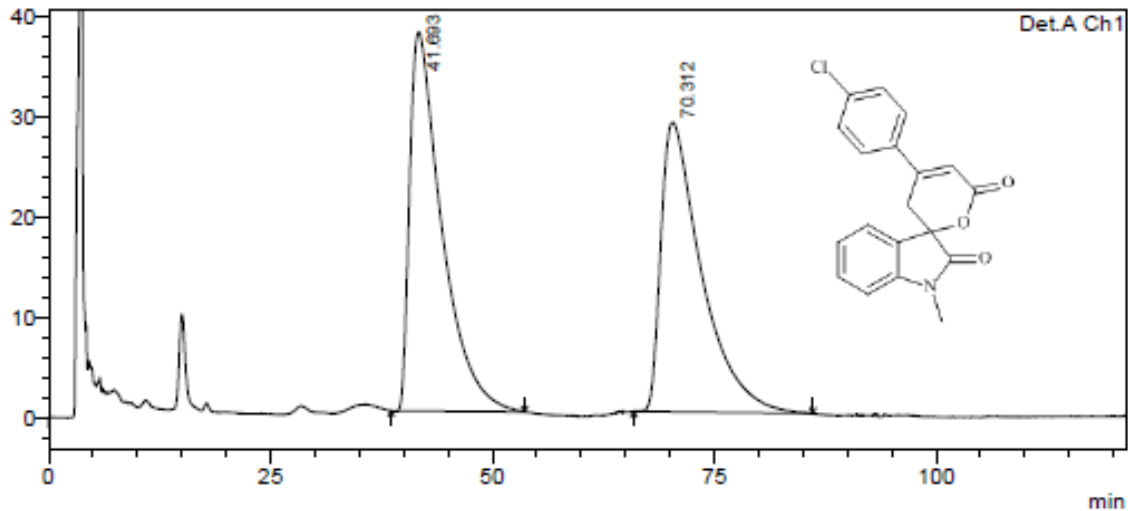

\begin{tabular}{|r|r|r|r|r|r|}
\hline \multicolumn{1}{|c|}{ Pealk } & Ret. Time & \multicolumn{1}{c|}{ Area } & Height & \multicolumn{1}{c|}{ Area \% } & \multicolumn{1}{c|}{ Height \% } \\
\hline 1 & 41.693 & 9523620 & 37827 & 50.906 & 56.697 \\
\hline 2 & 70.312 & 9184662 & 28891 & 49.094 & 43.303 \\
\hline Total & & 18708281 & 66718 & 100.000 & 100.000 \\
\hline
\end{tabular}

$\mathrm{mV}$

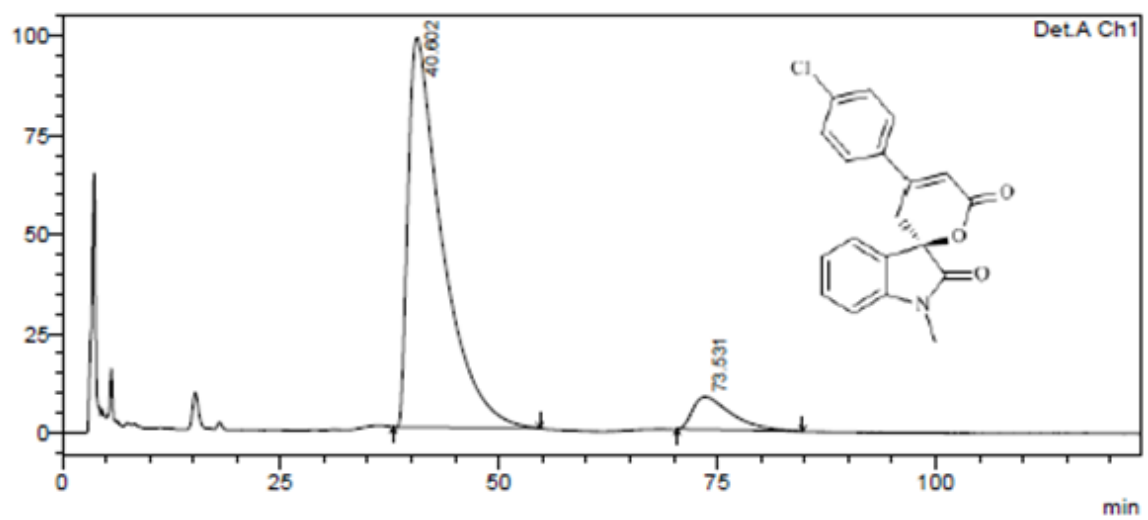

\begin{tabular}{|r|r|r|r|r|r|}
\hline \multicolumn{1}{|c|}{ Peak= } & \multicolumn{1}{|c|}{ Ret. Time } & \multicolumn{1}{c|}{ Area } & \multicolumn{1}{c|}{ Height } & \multicolumn{1}{c|}{ Area \% } & \multicolumn{1}{c|}{ Height \% } \\
\hline 1 & 40.602 & 26572084 & 98140 & 91.288 & 92.250 \\
\hline 2 & 73.531 & 2535748 & 8245 & 8.712 & 7.750 \\
\hline Total & & 29107832 & 106385 & 100.000 & 100.000 \\
\hline
\end{tabular}


(R)-4'-(4-bromophenyl)-1-methylspiro[indoline-3,2'-pyran]-2,6'(3'H)-dione（3f) $\mathrm{mV}$

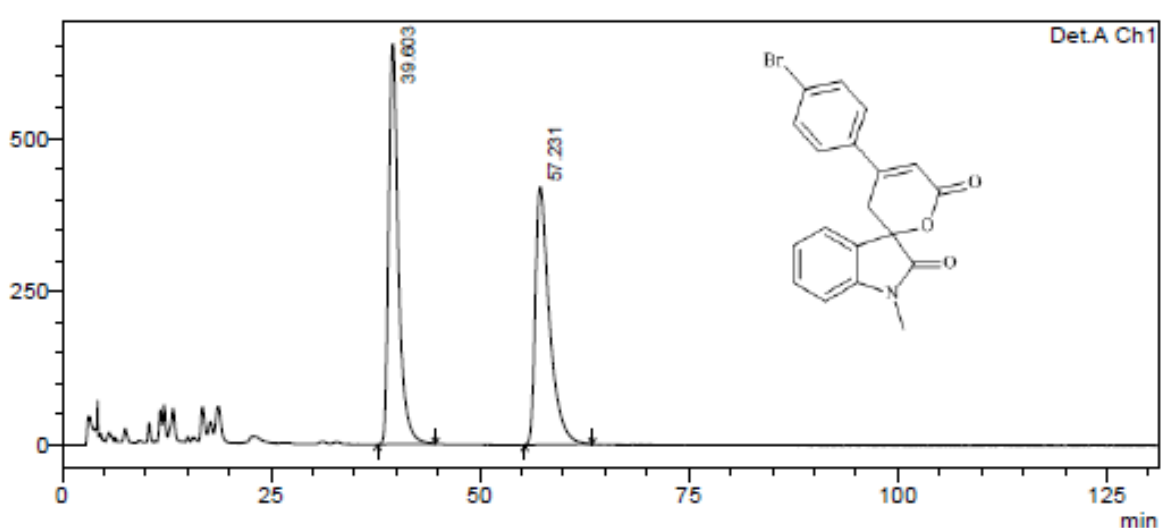

\begin{tabular}{|r|r|r|r|r|r|}
\hline \multicolumn{1}{|c|}{ Peals } & \multicolumn{1}{|c|}{ Ret. Time } & \multicolumn{1}{c|}{ Area } & \multicolumn{1}{c|}{ Height } & \multicolumn{1}{c|}{ Area \% } & \multicolumn{1}{c|}{ Height \% } \\
\hline 1 & 39.603 & 52818586 & 651877 & 51.651 & 60.833 \\
\hline 2 & 57.231 & 49442045 & 419705 & 48.349 & 39.167 \\
\hline Total & & 102260630 & 1071582 & 100.000 & 100.000 \\
\hline
\end{tabular}

$\mathrm{mV}$

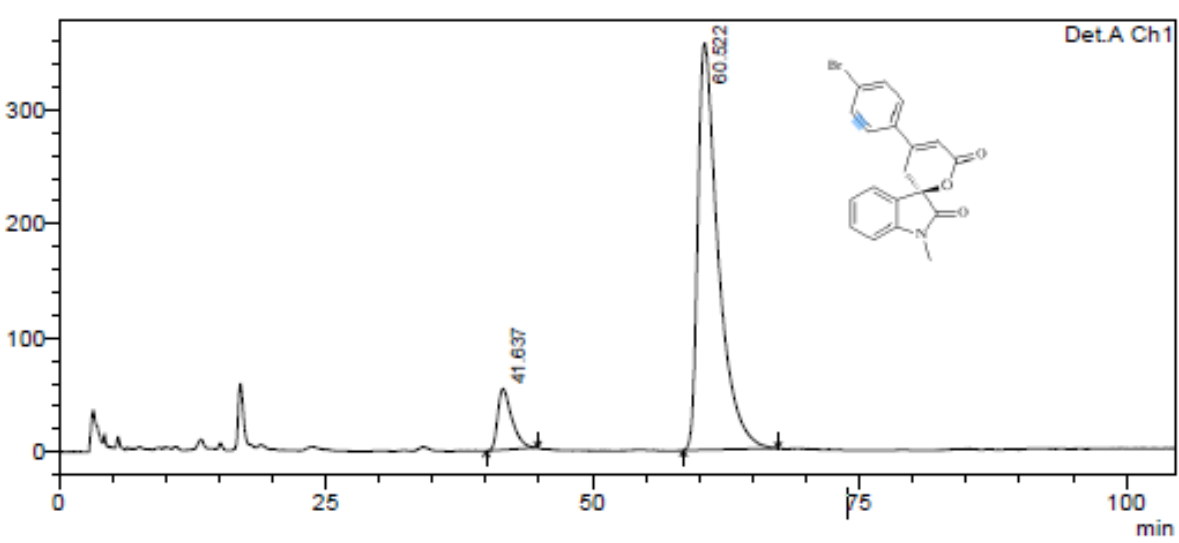

\begin{tabular}{|r|r|r|r|r|r|}
\hline \multicolumn{1}{|c|}{ Peak" } & Ret. Time & \multicolumn{1}{c|}{ Area } & \multicolumn{1}{c|}{ Height } & Area \% & \multicolumn{1}{c|}{ Height \% } \\
\hline 1 & 41.637 & 4952422 & 53386 & 9.425 & 13.022 \\
\hline 2 & 60.522 & 47593544 & 356572 & 90.575 & 86.978 \\
\hline Tota & & 52545966 & 409959 & 100.000 & 100.000 \\
\hline
\end{tabular}


(R)-4'-(furan-2-yl)-1-methylspiro[indoline-3,2'-pyran]-2,6' (3'H)-dione (3g) $\mathrm{mV}$

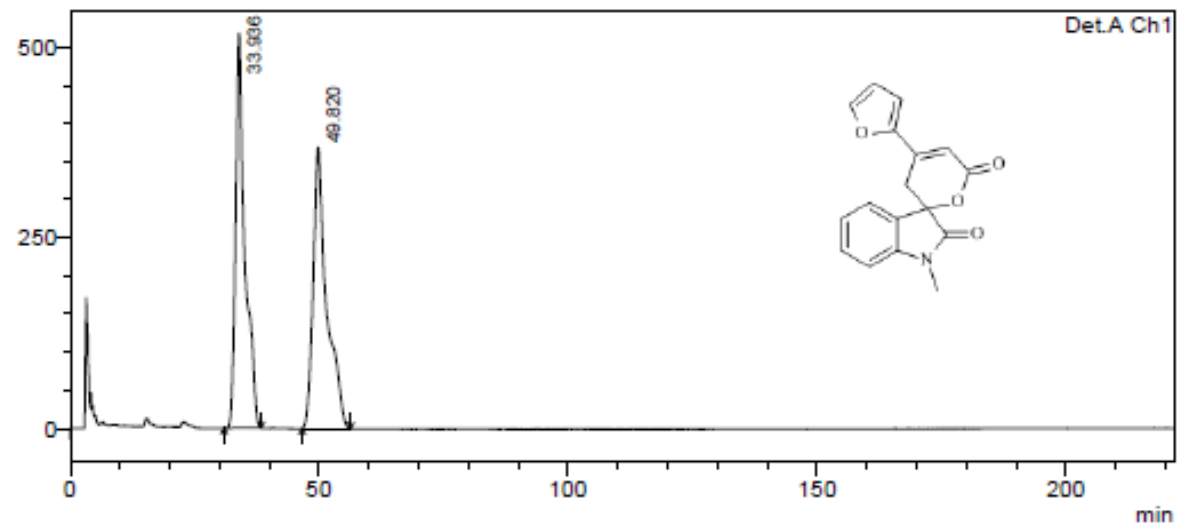

\begin{tabular}{|r|r|r|r|r|r|}
\hline \multicolumn{1}{|c|}{ Peak $\#$} & Ret. Time & \multicolumn{1}{c|}{ Area } & Height & \multicolumn{1}{c|}{ Area \% } & \multicolumn{1}{c|}{ Height \% } \\
\hline 1 & 33.936 & 66867074 & 517206 & 49.035 & 58.407 \\
\hline 2 & 49.820 & 69498528 & 368308 & 50.965 & 41.593 \\
\hline Tota. & & 136365602 & 885514 & 100.000 & 100.000 \\
\hline
\end{tabular}

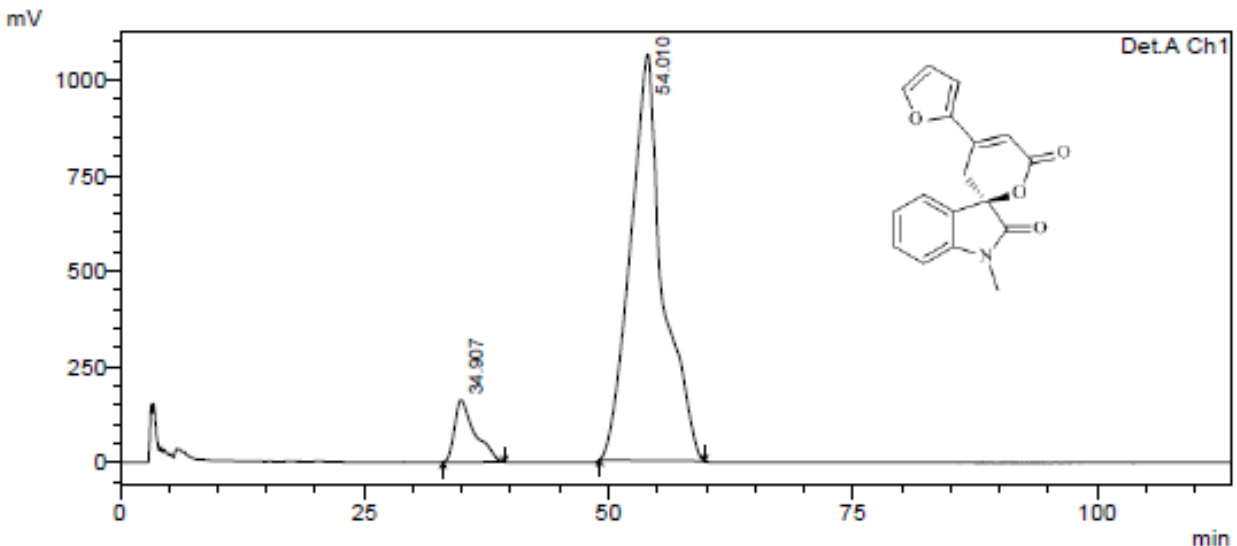

\begin{tabular}{|r|r|r|r|r|r|}
\hline \multicolumn{1}{|c|}{ Pealk } & Ret. Time & \multicolumn{1}{c|}{ Area } & \multicolumn{1}{c|}{ Height } & Area \% & \multicolumn{1}{c|}{ Height \% } \\
\hline 1 & 34.907 & 23548476 & 162627 & 8.733 & 13.287 \\
\hline 2 & 54.010 & 246095253 & 1061308 & 91.267 & 86.713 \\
\hline Total & & 269643728 & 1223935 & 100.000 & 100.000 \\
\hline
\end{tabular}


(R)-4'-cyclohexyl-1-methylspiro[indoline-3,2'-pyran]-2,6'(3'H)-dione（3h $)$ $\mathrm{mV}$

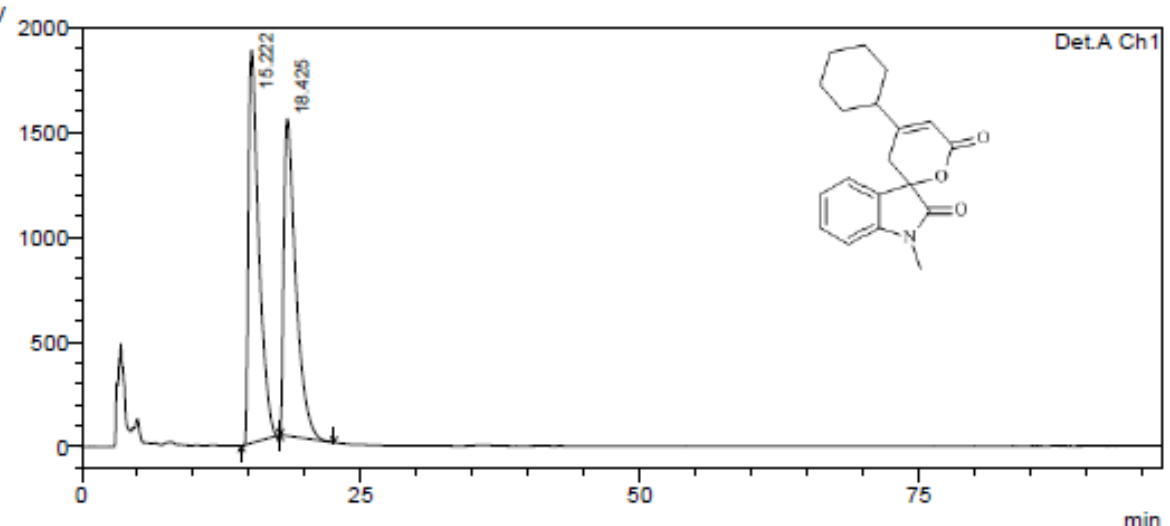

\begin{tabular}{|r|r|r|r|r|r|}
\hline \multicolumn{1}{|c|}{ Pealf } & Ret. Time & \multicolumn{1}{c|}{ Area } & \multicolumn{1}{c|}{ Height } & \multicolumn{1}{c|}{ Area \% } & \multicolumn{1}{c|}{ Height \% } \\
\hline 1 & 15.222 & 120407337 & 1876997 & 51.594 & 55.356 \\
\hline 2 & 18.425 & 112968527 & 1513778 & 48.406 & 44.644 \\
\hline Total & & 233375864 & 3390775 & 100.000 & 100.000 \\
\hline
\end{tabular}

$\mathrm{mV}$

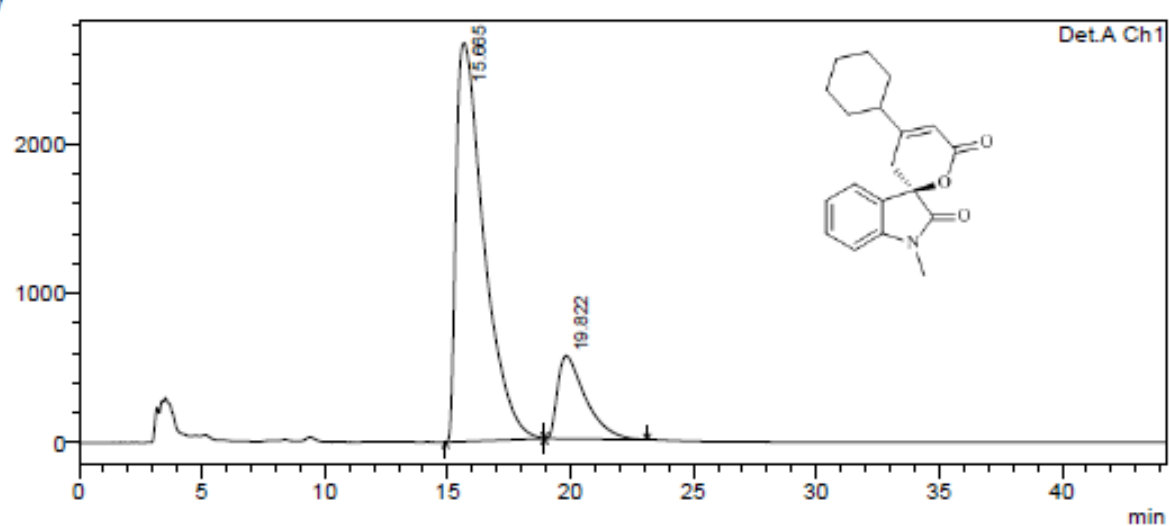

\begin{tabular}{|r|r|r|r|r|r|}
\hline \multicolumn{1}{|c|}{ Pealik } & \multicolumn{1}{|c|}{ Ret. Time } & \multicolumn{1}{c|}{ Area } & \multicolumn{1}{c|}{ Height } & \multicolumn{1}{c|}{ Area \% } & \multicolumn{1}{c|}{ Height \% } \\
\hline 1 & 15.665 & 209971477 & 2666947 & 82.749 & 82.778 \\
\hline 2 & 19.822 & 43772256 & 554844 & 17.251 & 17.222 \\
\hline Total & & 253743733 & 3221791 & 100.000 & 100.000 \\
\hline
\end{tabular}


(R)-1,4'-dimethylspiro[indoline-3,2'-pyran]-2,6'(3'H)-dione（3i $)$

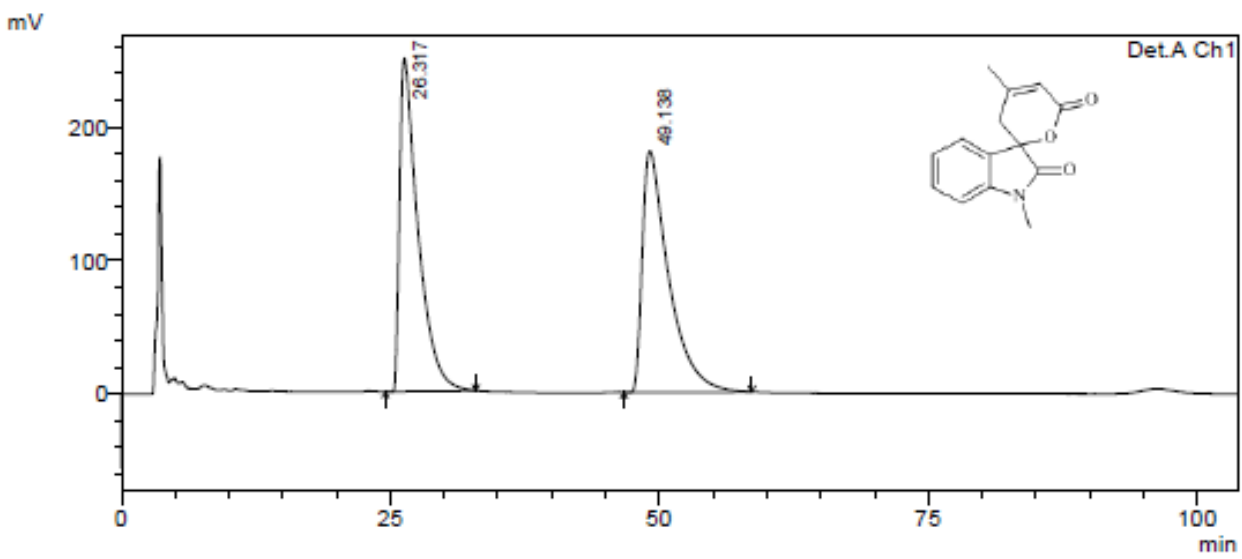

\begin{tabular}{|r|r|r|r|r|r|}
\hline \multicolumn{1}{|c|}{ Peak\# } & \multicolumn{1}{|c|}{ Ret. Time } & \multicolumn{1}{c|}{ Area } & \multicolumn{1}{c|}{ Height } & \multicolumn{1}{c|}{ Area \% } & \multicolumn{1}{c|}{ Height \% } \\
\hline 1 & 26.317 & 29766071 & 249534 & 49.164 & 58.016 \\
\hline 2 & 49.138 & 30778271 & 180575 & 50.836 & 41.984 \\
\hline Total & & 60544342 & 430109 & 100.000 & 100.000 \\
\hline
\end{tabular}

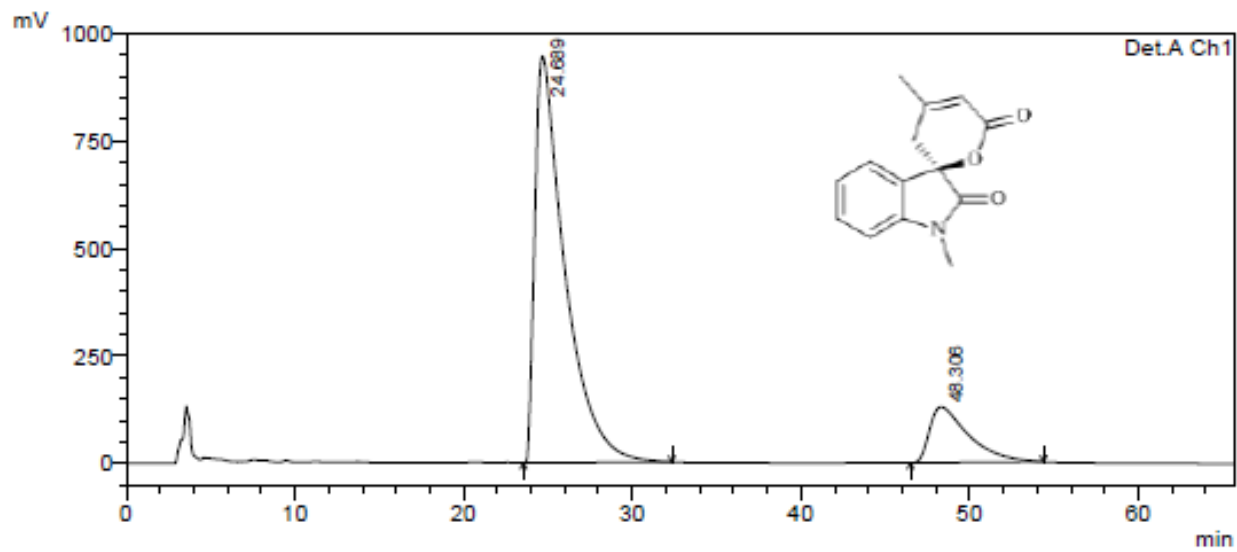

\begin{tabular}{|r|r|r|r|r|r|}
\hline \multicolumn{1}{|c|}{ Peal $=$} & \multicolumn{1}{|c|}{ Ret. Time } & \multicolumn{1}{c|}{ Area } & \multicolumn{1}{c|}{ Height } & \multicolumn{1}{c|}{ Area \% } & \multicolumn{1}{c|}{ Height \% } \\
\hline 1 & 24.689 & 117097362 & 945202 & 84.841 & 87.940 \\
\hline 2 & 48.306 & 20921848 & 129628 & 15.159 & 12.060 \\
\hline Total & & 138019210 & 1074830 & 100.000 & 100.000 \\
\hline
\end{tabular}


(R)-6-chloro-1-methyl-4'-phenylspiro[indoline-3,2'-pyran]-2,6'(3'H)-dione（3j)

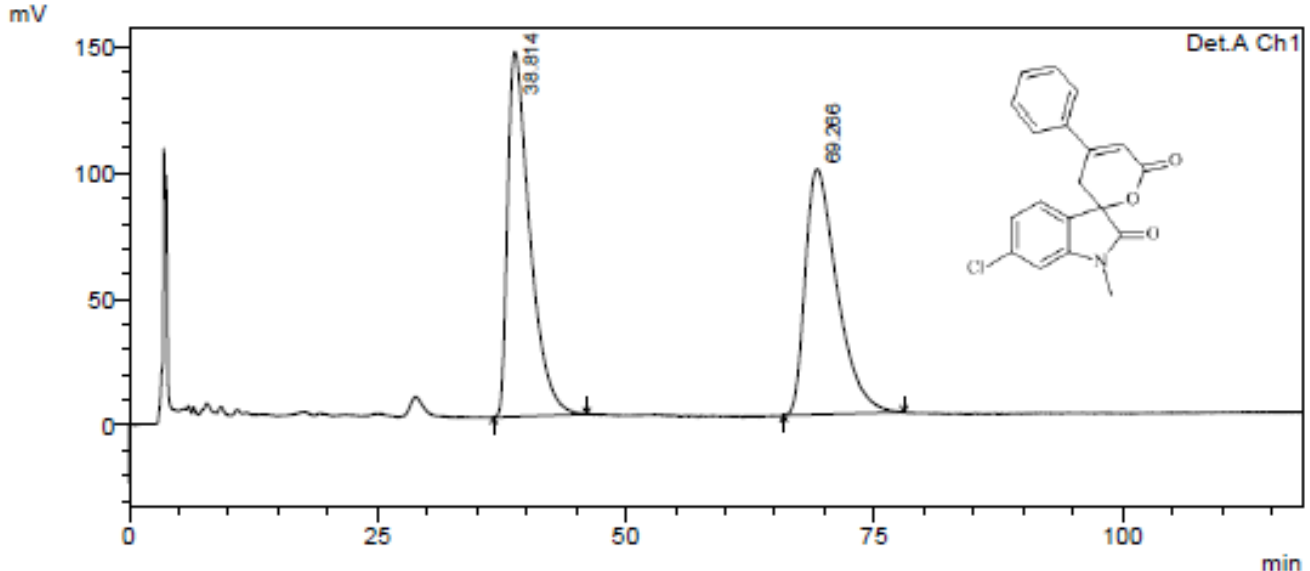

\begin{tabular}{|r|r|r|r|r|r|}
\hline \multicolumn{1}{|c|}{ Peak $=$} & Ret. Time & \multicolumn{1}{|c|}{ Area } & \multicolumn{1}{c|}{ Height } & \multicolumn{1}{c|}{ Area \% } & \multicolumn{1}{c|}{ Height \% } \\
\hline 1 & 38.814 & 22572505 & 144833 & 50.924 & 59.792 \\
\hline 2 & 69.266 & 21753365 & 97394 & 49.076 & 40.208 \\
\hline Tota: & & 44325870 & 242227 & 100.000 & 100.000 \\
\hline
\end{tabular}

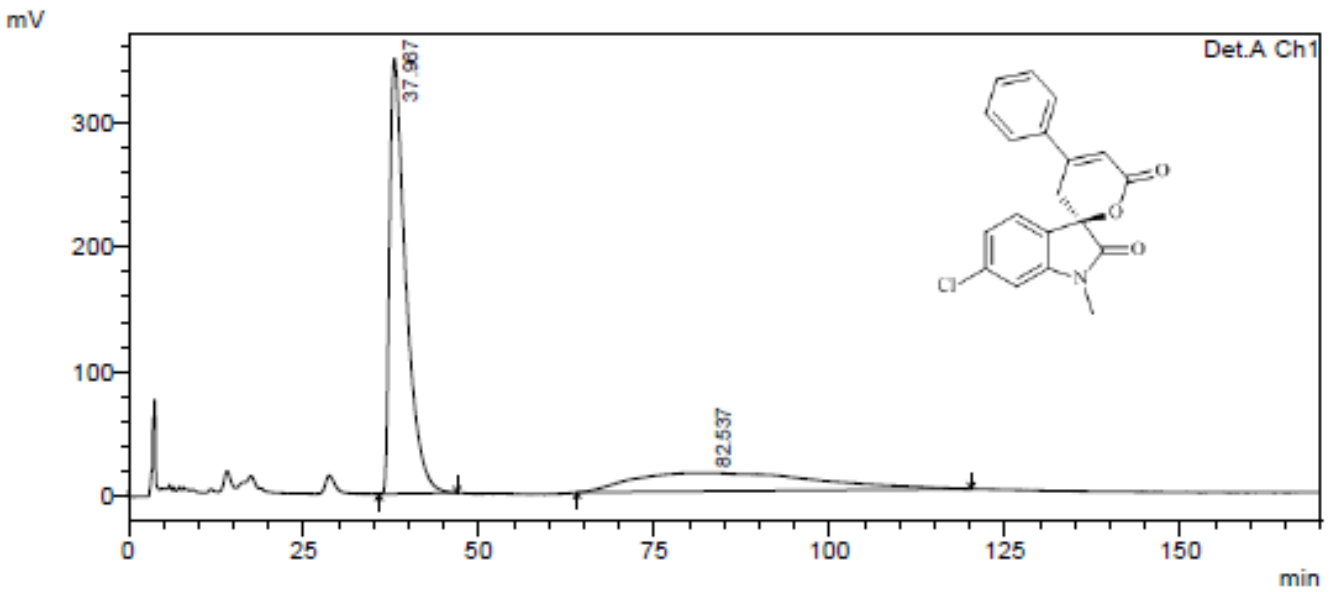

\begin{tabular}{|r|r|r|r|r|r|}
\hline \multicolumn{1}{|c|}{ Pealk } & Ret. Time & \multicolumn{1}{|c|}{ Area } & \multicolumn{1}{c|}{ Height } & \multicolumn{1}{c|}{ Area \% } & \multicolumn{1}{c|}{ Height \% } \\
\hline 1 & 37.967 & 56044351 & 348323 & 67.488 & 95.923 \\
\hline 2 & 82.537 & 26999556 & 14804 & 32.512 & 4.077 \\
\hline Total & & 83043907 & 363127 & 100.000 & 100.000 \\
\hline
\end{tabular}


(R)-6-chloro-4'-(4-methoxyphenyl)-1-methylspiro[indoline-3,2'-pyran]-2,6'(3'H)-dion e $(3 \mathbf{k})$

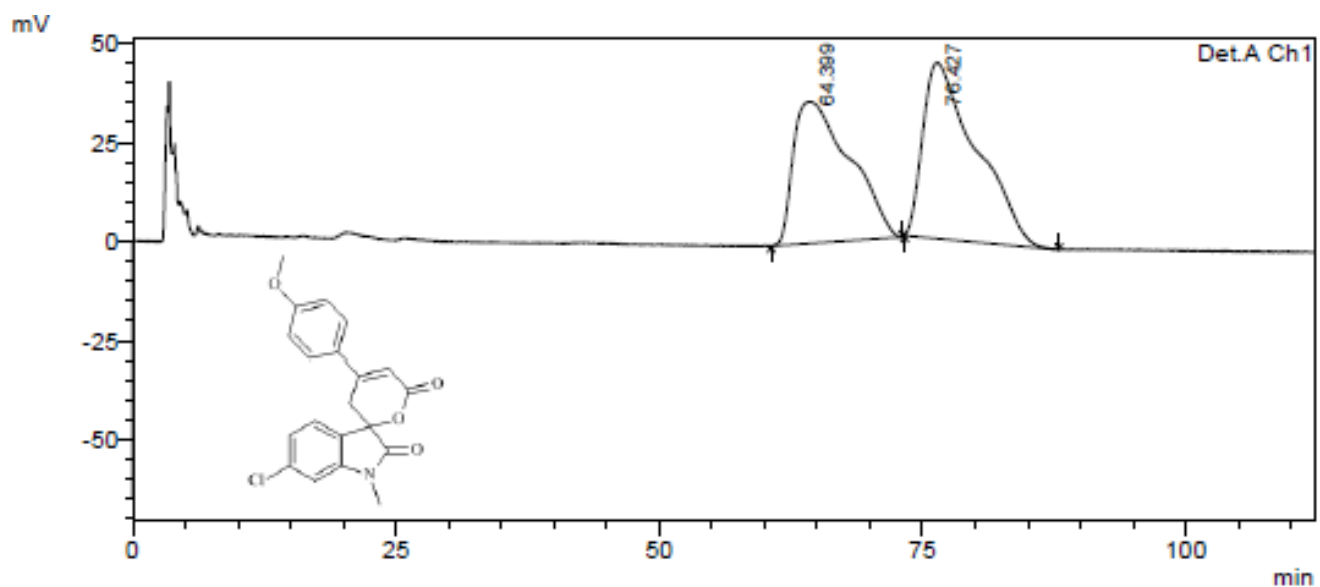

\begin{tabular}{|r|r|r|r|r|r|}
\hline \multicolumn{1}{|c|}{ Pealk\# } & Ret. Time & \multicolumn{1}{|c|}{ Area } & \multicolumn{1}{c|}{ Height } & \multicolumn{1}{c|}{ Area \% } & \multicolumn{1}{c|}{ Height \% } \\
\hline 1 & 64.399 & 13266695 & 35678 & 45.920 & 44.537 \\
\hline 2 & 76.427 & 15624117 & 44430 & 54.080 & 55.463 \\
\hline Total & & 28890812 & 80108 & 100.000 & 100.000 \\
\hline
\end{tabular}

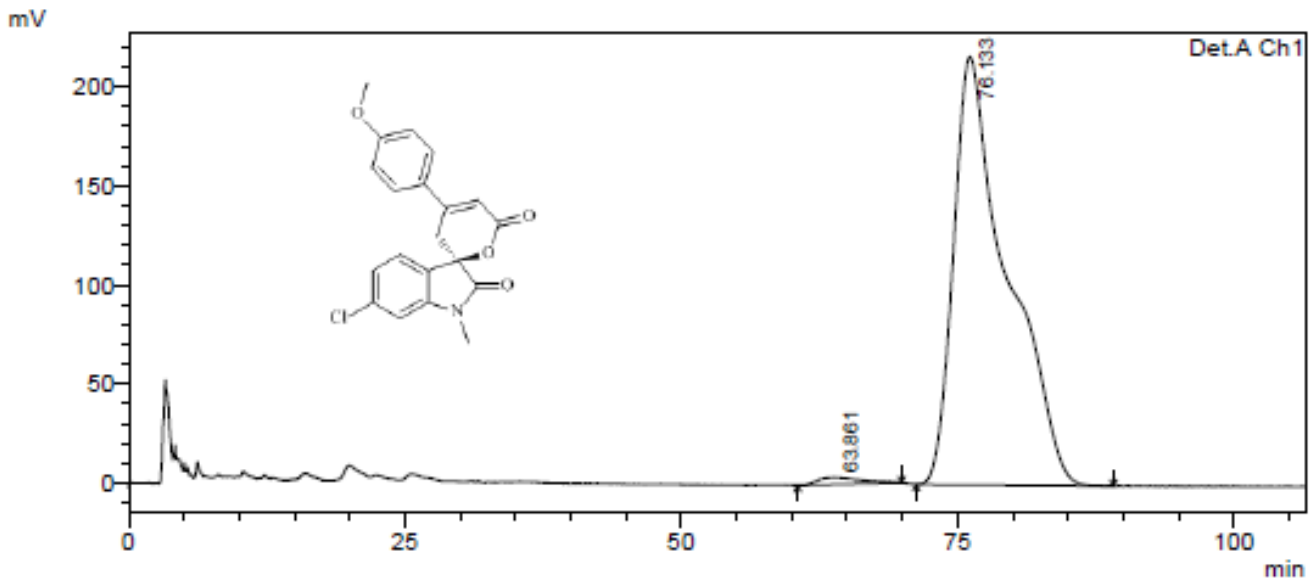

\begin{tabular}{|r|r|r|r|r|r|}
\hline \multicolumn{1}{|c|}{ Pealk\# } & Ret. Time & \multicolumn{1}{c|}{ Area } & \multicolumn{1}{c|}{ Height } & \multicolumn{1}{c|}{ Area \% } & \multicolumn{1}{c|}{ Height \% } \\
\hline 1 & 63.861 & 954631 & 3627 & 1.294 & 1.652 \\
\hline 2 & 76.133 & 72815067 & 215865 & 98.706 & 98.348 \\
\hline Total & & 73769698 & 219492 & 100.000 & 100.000 \\
\hline
\end{tabular}


(R)-6-chloro-4'-(4-fluorophenyl)-1-methylspiro[indoline-3,2'-pyran]-2,6'(3'H)-dione (31)

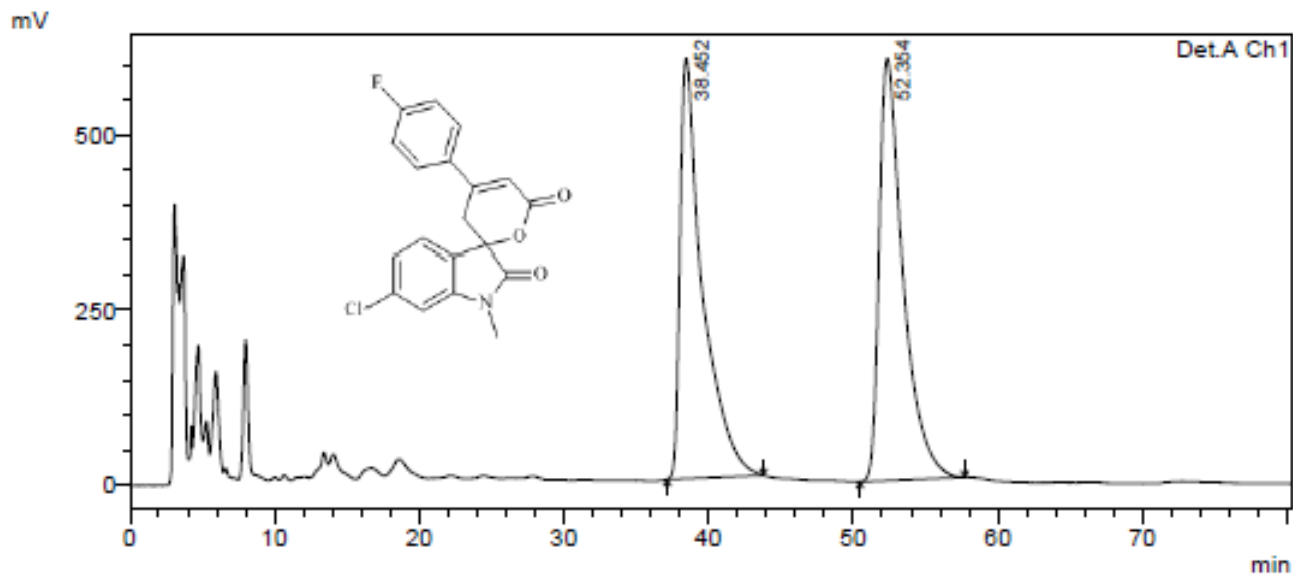

\begin{tabular}{|r|r|r|r|r|r|}
\hline \multicolumn{1}{|c|}{ Pealk $=$} & Ret. Time & \multicolumn{1}{c|}{ Area } & Height & \multicolumn{1}{c|}{ Area \% } & \multicolumn{1}{c|}{ Height \% } \\
\hline 1 & 38.452 & 64937324 & 599429 & 48.423 & 49.891 \\
\hline 2 & 52.354 & 69165868 & 602054 & 51.577 & 50.109 \\
\hline Total & & 134103192 & 1201483 & 100.000 & 100.000 \\
\hline
\end{tabular}

$\mathrm{mV}$

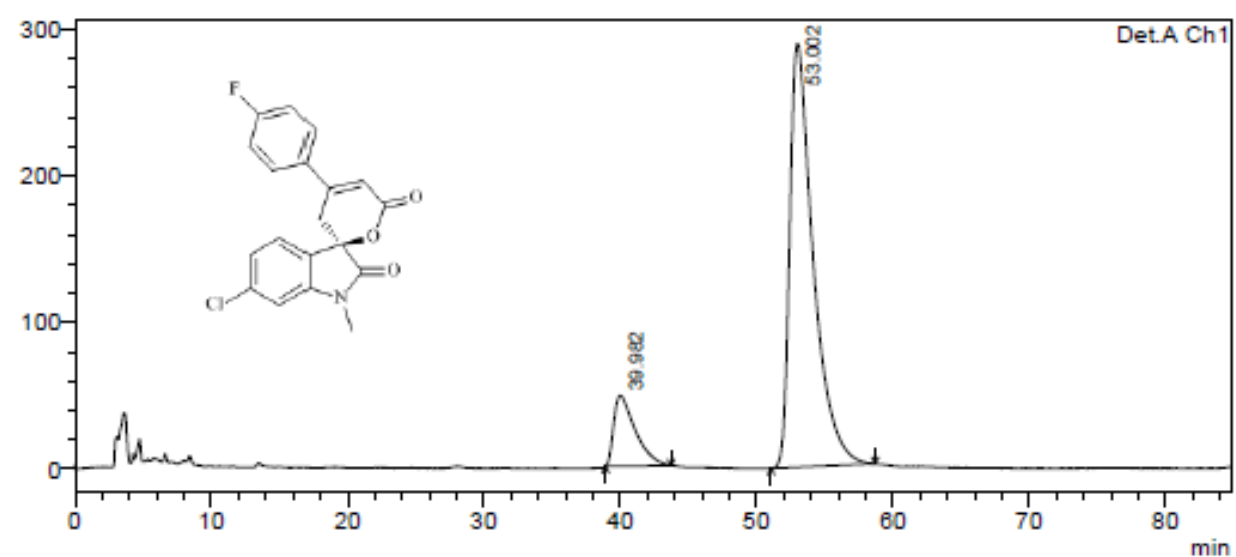

\begin{tabular}{|r|r|r|r|r|r|}
\hline Peak $=$ & Ret. Time & \multicolumn{1}{c|}{ Area } & Height & \multicolumn{1}{c|}{ Area \% } & \multicolumn{1}{c|}{ Height \% } \\
\hline 1 & 39.982 & 5275771 & 48186 & 13.228 & 14.287 \\
\hline 2 & 53.002 & 34606436 & 289086 & 86.772 & 85.713 \\
\hline Total & & 39882206 & 337272 & 100.000 & 100.000 \\
\hline
\end{tabular}


(R)-4'-(4-bromophenyl)-6-chloro-1-methylspiro[indoline-3,2'-pyran]-2,6'(3'H)-di one (3m)

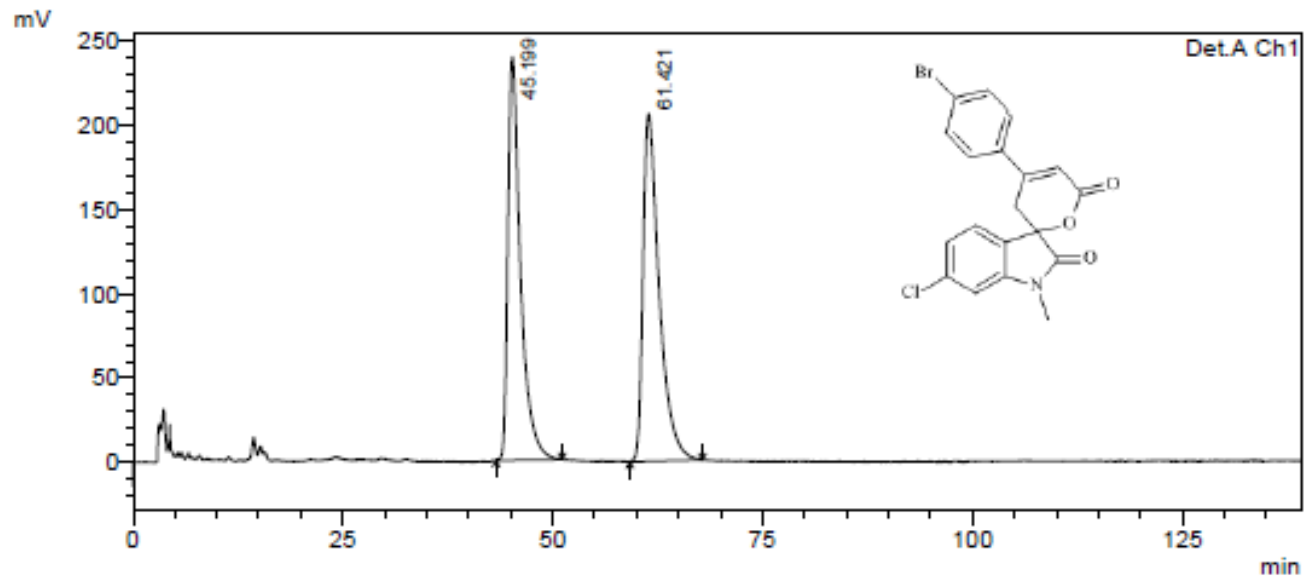

\begin{tabular}{|r|r|r|r|r|r|}
\hline Peak\# & Ret. Time & \multicolumn{1}{|c|}{ Area } & \multicolumn{1}{c|}{ Height } & \multicolumn{1}{c|}{ Area \% } & \multicolumn{1}{c|}{ Height \% } \\
\hline 1 & 45.199 & 25140623 & 239447 & 47.603 & 53.704 \\
\hline 2 & 61.421 & 27672489 & 206419 & 52.397 & 46.296 \\
\hline Total & & 52813112 & 445866 & 100.000 & 100.000 \\
\hline
\end{tabular}

$\mathrm{mV}$

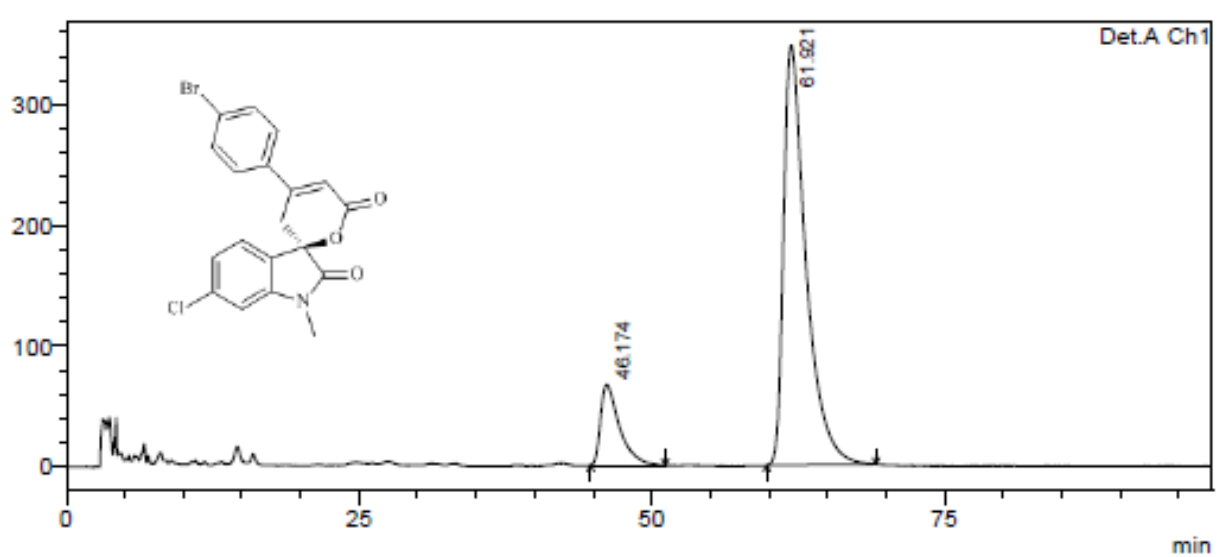

\begin{tabular}{|r|r|r|r|r|r|}
\hline \multicolumn{1}{|c|}{ Peak\# } & Ret. Time & \multicolumn{1}{c|}{ Area } & \multicolumn{1}{c|}{ Height } & \multicolumn{1}{c|}{ Area \% } & Height \% \\
\hline 1 & 46.174 & 7456477 & 66721 & 13.904 & 16.109 \\
\hline 2 & 61.921 & 46171538 & 347461 & 86.096 & 83.891 \\
\hline Total & & 53628015 & 414182 & 100.000 & 100.000 \\
\hline
\end{tabular}


(R)-6-chloro-1,4'-dimethylspiro[indoline-3,2'-pyran]-2,6'(3'H)-dione（3n $\mathrm{mV}$

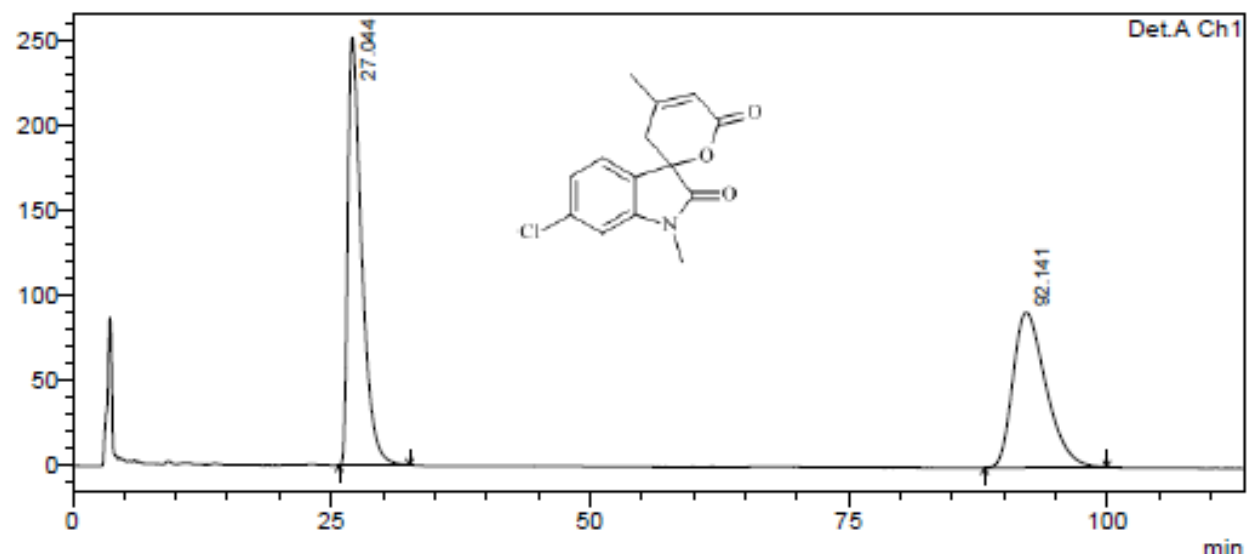

\begin{tabular}{|r|r|r|r|r|r|}
\hline \multicolumn{1}{|c|}{ Peak\# } & \multicolumn{1}{|c|}{ Ret. Time } & \multicolumn{1}{c|}{ Area } & \multicolumn{1}{c|}{ Height } & \multicolumn{1}{c|}{ Area \% } & \multicolumn{1}{c|}{ Height \% } \\
\hline 1 & 27.044 & 23452154 & 251151 & 53.600 & 73.356 \\
\hline 2 & 92.141 & 20302231 & 91220 & 46.400 & 26.644 \\
\hline Total & & 43754385 & 342371 & 100.000 & 100.000 \\
\hline
\end{tabular}

$\mathrm{mV}$

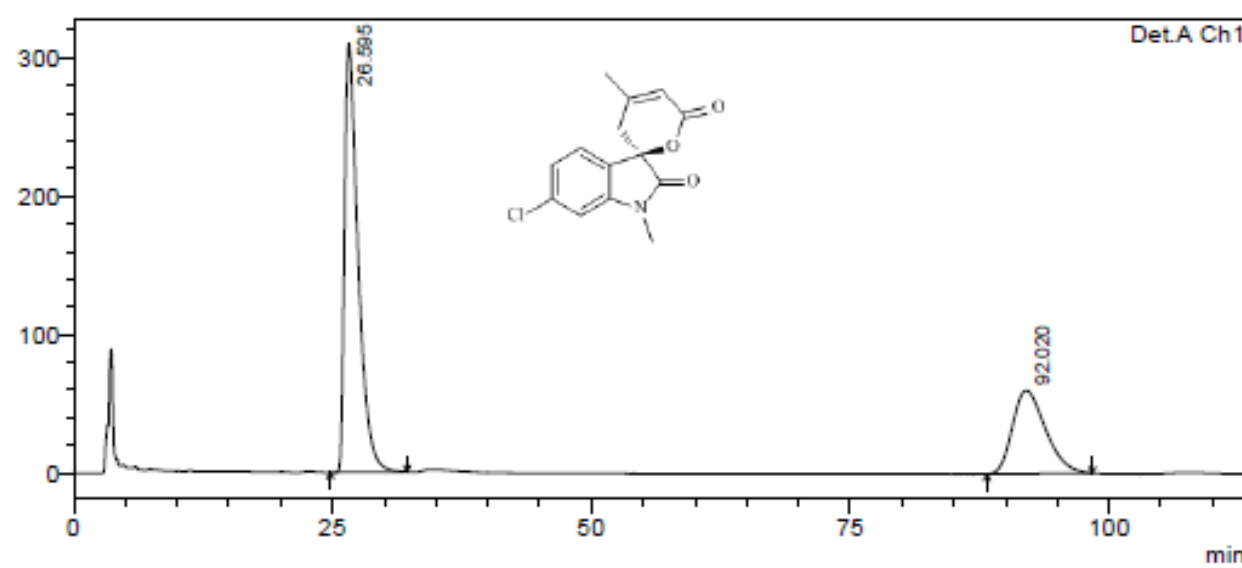

\begin{tabular}{|r|r|r|r|r|r|}
\hline \multicolumn{1}{|c|}{ Peaki } & Ret. Time & \multicolumn{1}{|c|}{ Area } & \multicolumn{1}{c|}{ Height } & \multicolumn{1}{c|}{ Area \% } & \multicolumn{1}{c|}{ Height \% } \\
\hline 1 & 26.595 & 28271901 & 309796 & 67.911 & 83.745 \\
\hline 2 & 92.020 & 13358957 & 60133 & 32.089 & 16.255 \\
\hline Total & & 41630858 & 369929 & 100.000 & 100.000 \\
\hline
\end{tabular}


(R)-6-bromo-4'-(4-bromophenyl)-1-methylspiro[indoline-3,2'-pyran]-2,6'(3'H)-di one $(30)$

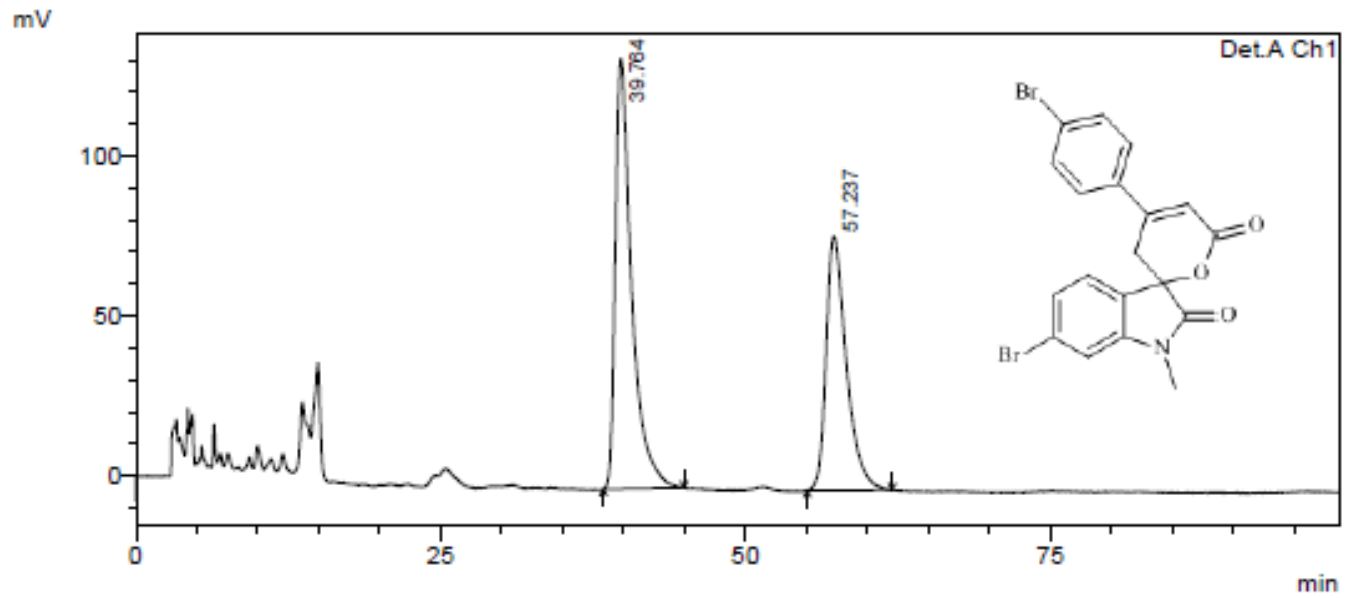

\begin{tabular}{|r|r|r|r|r|r|}
\hline \multicolumn{1}{|c|}{ Peal= } & Ret. Time & \multicolumn{1}{c|}{ Area } & \multicolumn{1}{c|}{ Height } & \multicolumn{1}{c|}{ Area \% } & Height \% \\
\hline 1 & 39.764 & 12571519 & 134515 & 57.810 & 62.875 \\
\hline 2 & 57.237 & 9174935 & 79427 & 42.190 & 37.125 \\
\hline Tota & & 21746453 & 213942 & 100.000 & 100.000 \\
\hline
\end{tabular}

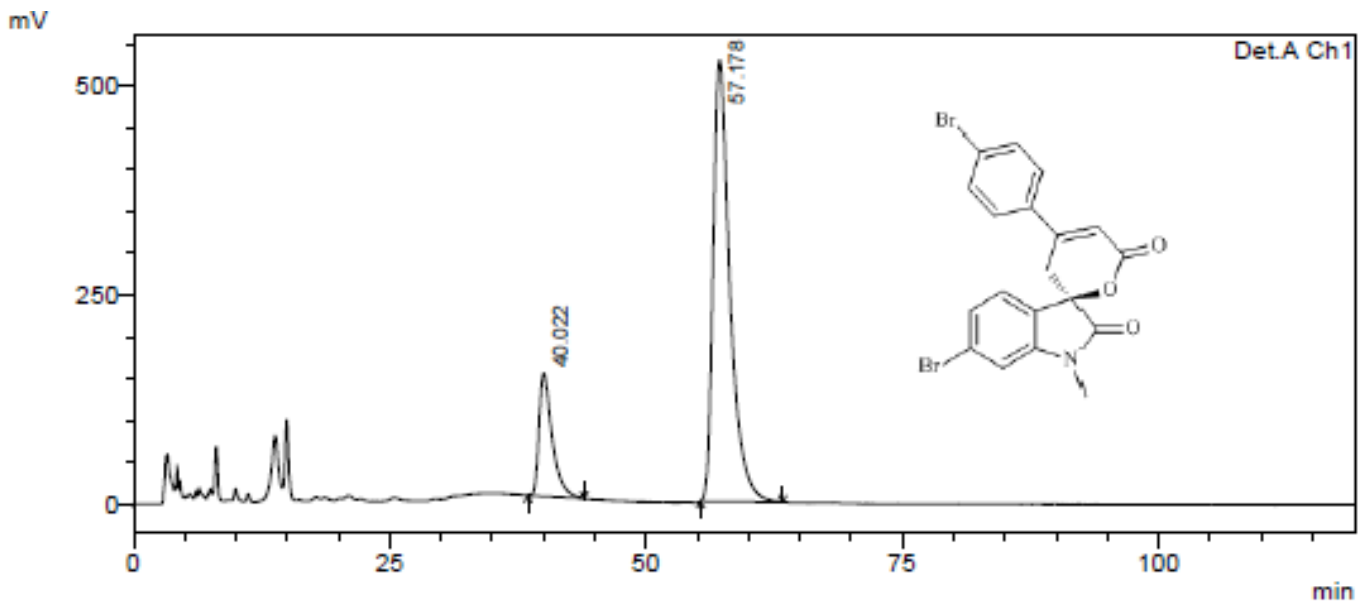

\begin{tabular}{|r|r|r|r|r|r|}
\hline \multicolumn{1}{|c|}{ Peak $\#$} & Ret. Time & \multicolumn{1}{c|}{ Area } & \multicolumn{1}{c|}{ Height } & \multicolumn{1}{c|}{ Area \% } & \multicolumn{1}{c|}{ Height \% } \\
\hline 1 & 40.022 & 13175760 & 147211 & 17.970 & 21.791 \\
\hline 2 & 57.178 & 60144165 & 528349 & 82.030 & 78.209 \\
\hline Tota & & 73319925 & 675559 & 100.000 & 100.000 \\
\hline
\end{tabular}


(R)-6-bromo-4'-(4-methoxyphenyl)-1-methylspiro[indoline-3,2'-pyran]-2,6' (3'H)dione (3p)

$\mathrm{mV}$

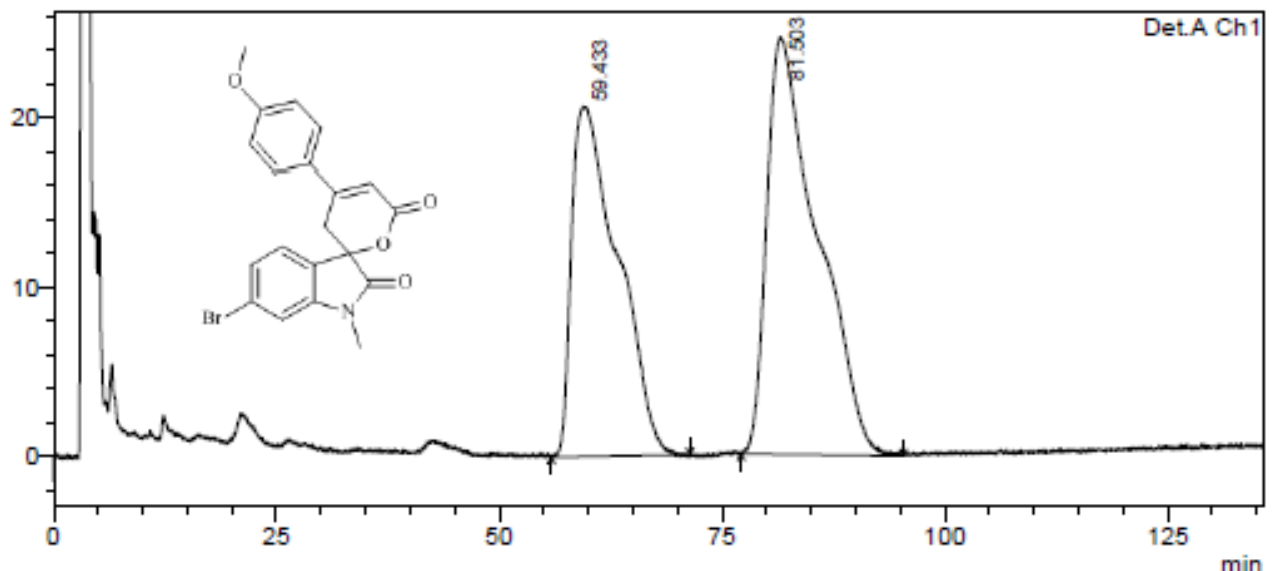

\begin{tabular}{|r|r|r|r|r|r|}
\hline \multicolumn{1}{|c|}{ Peali\# } & Ret. Time & \multicolumn{1}{c|}{ Area } & \multicolumn{1}{c|}{ Height } & Area \% & \multicolumn{1}{c|}{ Height \% } \\
\hline 1 & 59.433 & 7442943 & 20658 & 43.402 & 45.588 \\
\hline 2 & 81.503 & 9706081 & 24657 & 56.598 & 54.412 \\
\hline Total & & 17149024 & 45315 & 100.000 & 100.000 \\
\hline
\end{tabular}

$\mathrm{mV}$

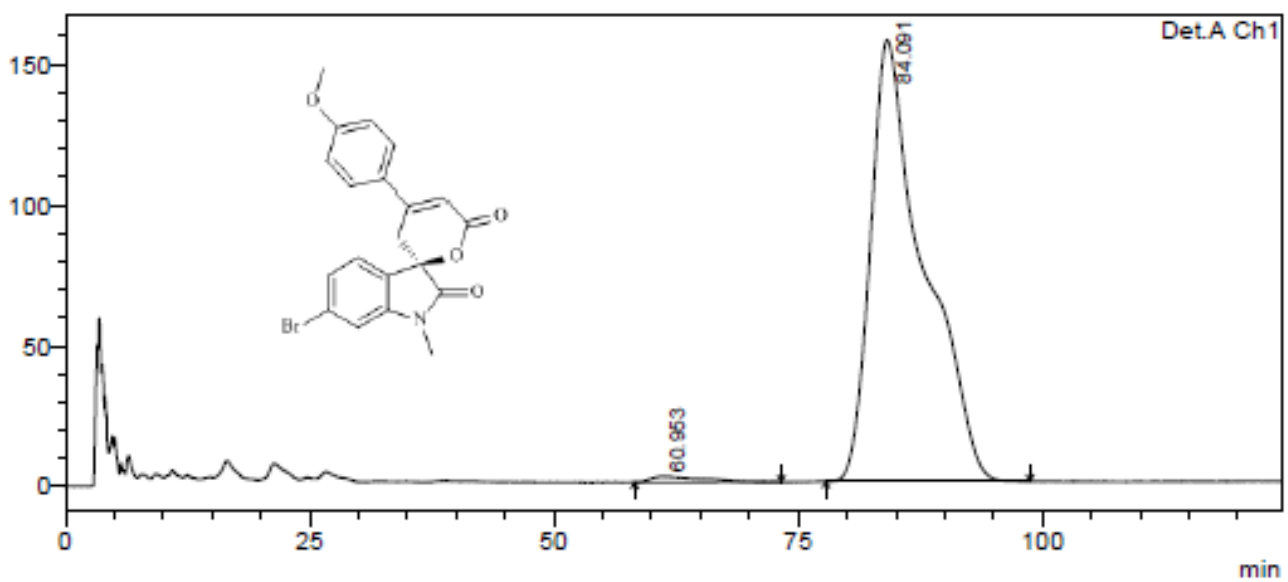

\begin{tabular}{|r|r|r|r|r|r|}
\hline \multicolumn{1}{|c|}{ Pealk\# } & Ret. Time & \multicolumn{1}{c|}{ Area } & \multicolumn{1}{c|}{ Height } & \multicolumn{1}{c|}{ Area \% } & Height \% \\
\hline 1 & 60.953 & 733848 & 2127 & 1.222 & 1.339 \\
\hline 2 & 84.091 & 59338105 & 156758 & 98.778 & 98.661 \\
\hline Total & & 60071954 & 158885 & 100.000 & 100.000 \\
\hline
\end{tabular}


(R)-6-methoxy-4'-(4-methoxyphenyl)-1-methylspiro[indoline-3,2'-pyran]-2,6'(3'

H)-dione (3q)

$\mathrm{mv}$

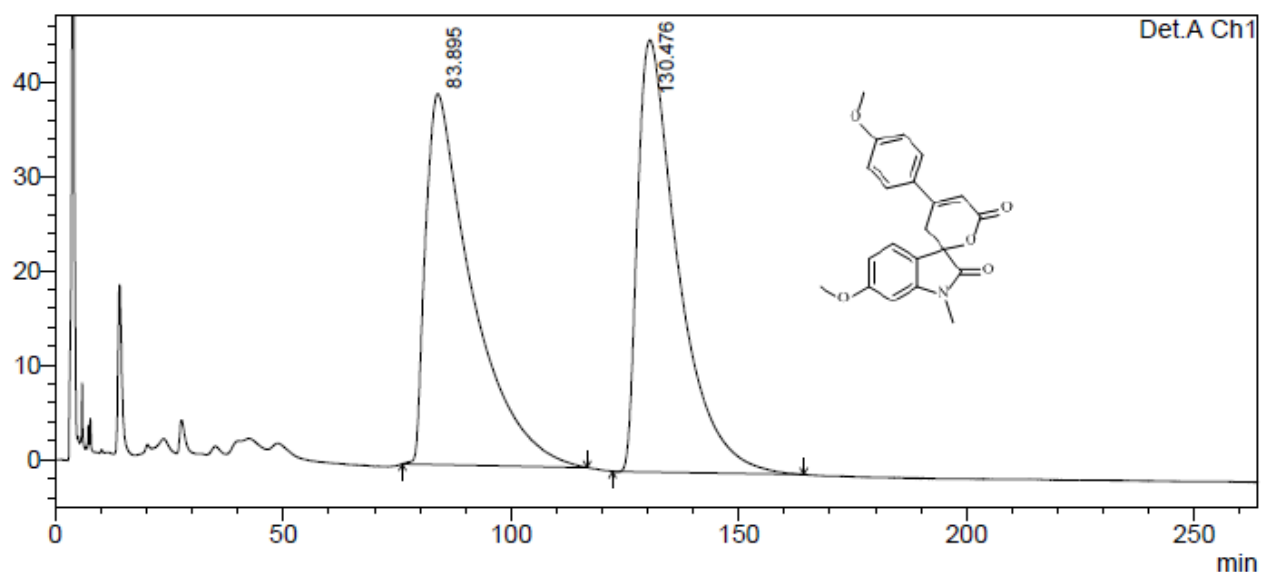

\begin{tabular}{|r|r|r|r|r|r|}
\hline \multicolumn{1}{|c|}{ Peak\# } & Ret. Time & \multicolumn{1}{c|}{ Area } & \multicolumn{1}{c|}{ Height } & Area \% & Height \% \\
\hline 1 & 83.895 & 27857147 & 39269 & 49.601 & 46.203 \\
\hline 2 & 130.476 & 28304865 & 45723 & 50.399 & 53.797 \\
\hline Tota. & & 56162012 & 84992 & 100.000 & 100.000 \\
\hline
\end{tabular}

$\mathrm{mV}$

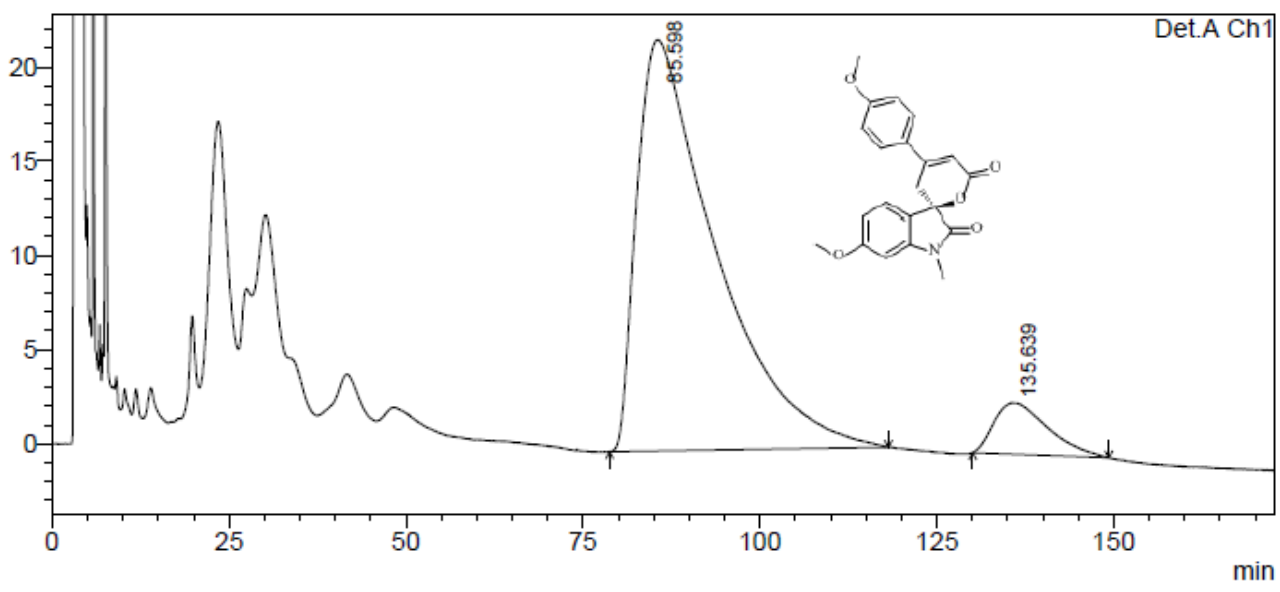

\begin{tabular}{|r|r|r|r|r|r|}
\hline \multicolumn{1}{|c|}{ Peak\# } & Ret. Time & \multicolumn{1}{|c|}{ Area } & \multicolumn{1}{c|}{ Height } & \multicolumn{1}{c|}{ Area \% } & Height \% \\
\hline 1 & 85.598 & 16870551 & 21825 & 92.121 & 88.882 \\
\hline 2 & 135.639 & 1442981 & 2730 & 7.879 & 11.118 \\
\hline Total & & 18313532 & 24555 & 100.000 & 100.000 \\
\hline
\end{tabular}


(R)-5-fluoro-4'-(4-methoxyphenyl)-1-methylspiro[indoline-3,2'-pyran]-2,6'(3'H)dione $(3 \mathbf{r})$

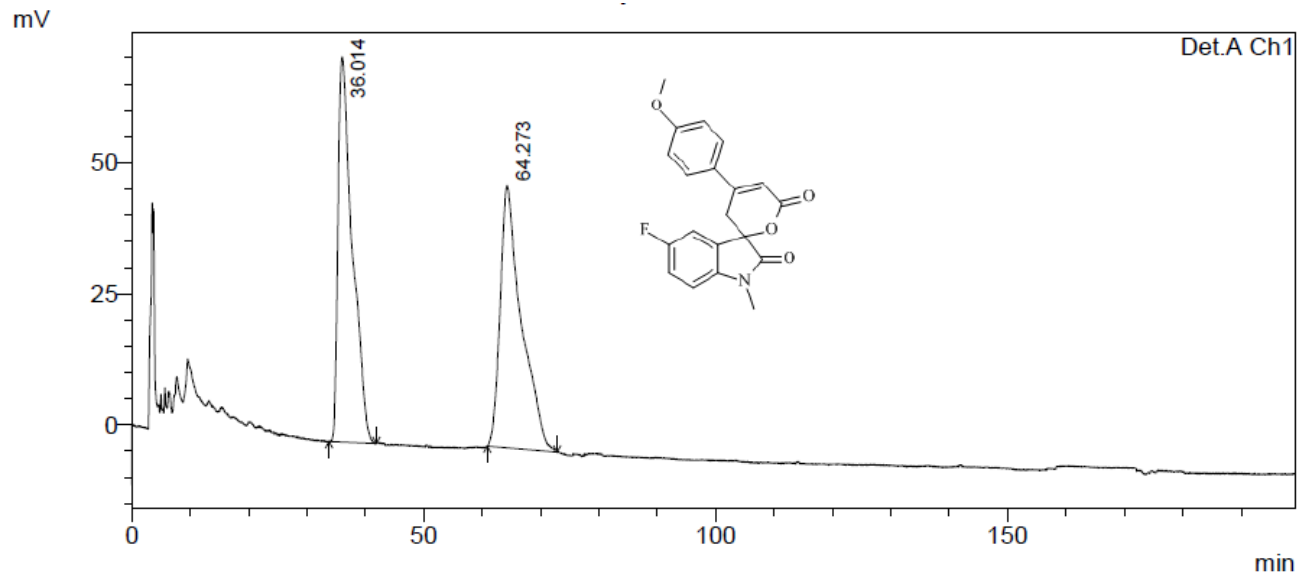

\begin{tabular}{|r|r|r|r|r|r|}
\hline Peak\# & Ret. Time & \multicolumn{1}{|c|}{ Area } & \multicolumn{1}{c|}{ Height } & \multicolumn{1}{c|}{ Area \% } & \multicolumn{1}{c|}{ Height \% } \\
\hline 1 & 36.014 & 12534832 & 73439 & 49.742 & 59.451 \\
\hline 2 & 64.273 & 12664935 & 50089 & 50.258 & 40.549 \\
\hline Total & & 25199766 & 123527 & 100.000 & 100.000 \\
\hline
\end{tabular}

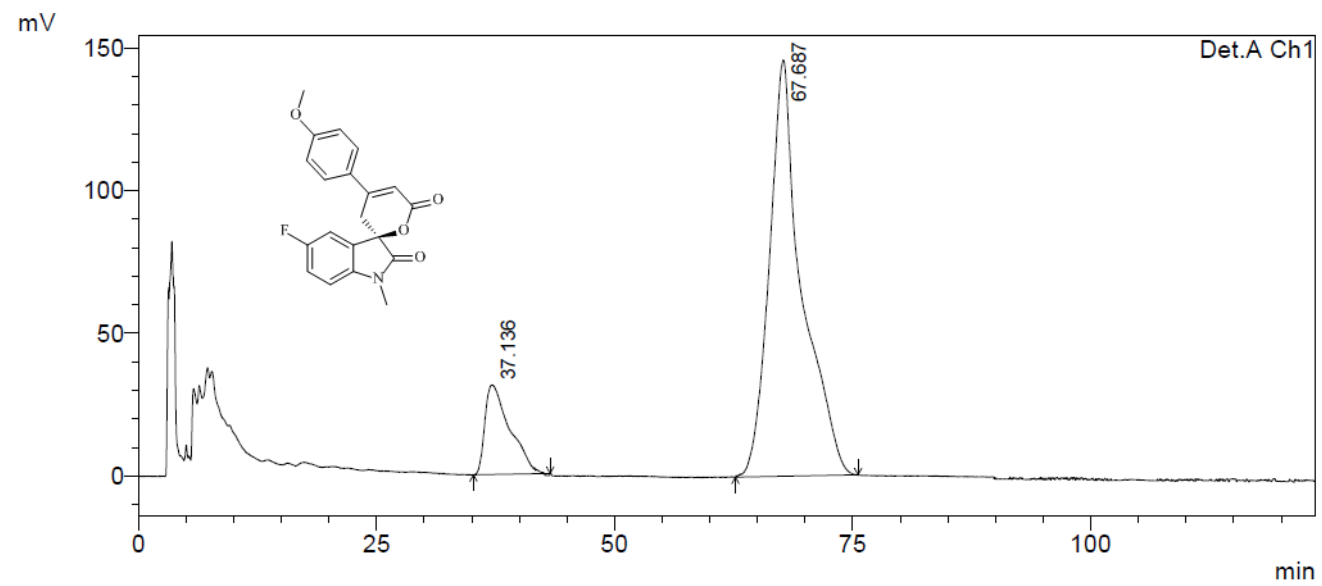

\begin{tabular}{|r|r|r|r|r|r|}
\hline Peak\# & Ret. Time & \multicolumn{1}{c|}{ Area } & \multicolumn{1}{c|}{ Height } & \multicolumn{1}{c|}{ Area \% } & \multicolumn{1}{c|}{ Height \% } \\
\hline 1 & 37.136 & 5688884 & 31367 & 14.117 & 17.696 \\
\hline 2 & 67.687 & 34610390 & 145885 & 85.883 & 82.304 \\
\hline Total & & 40299275 & 177252 & 100.000 & 100.000 \\
\hline
\end{tabular}


(R)-5-chloro-4'-(4-methoxyphenyl)-1-methylspiro[indoline-3,2'-pyran]-2,6'(3'H)dione $(3 \mathbf{s})$

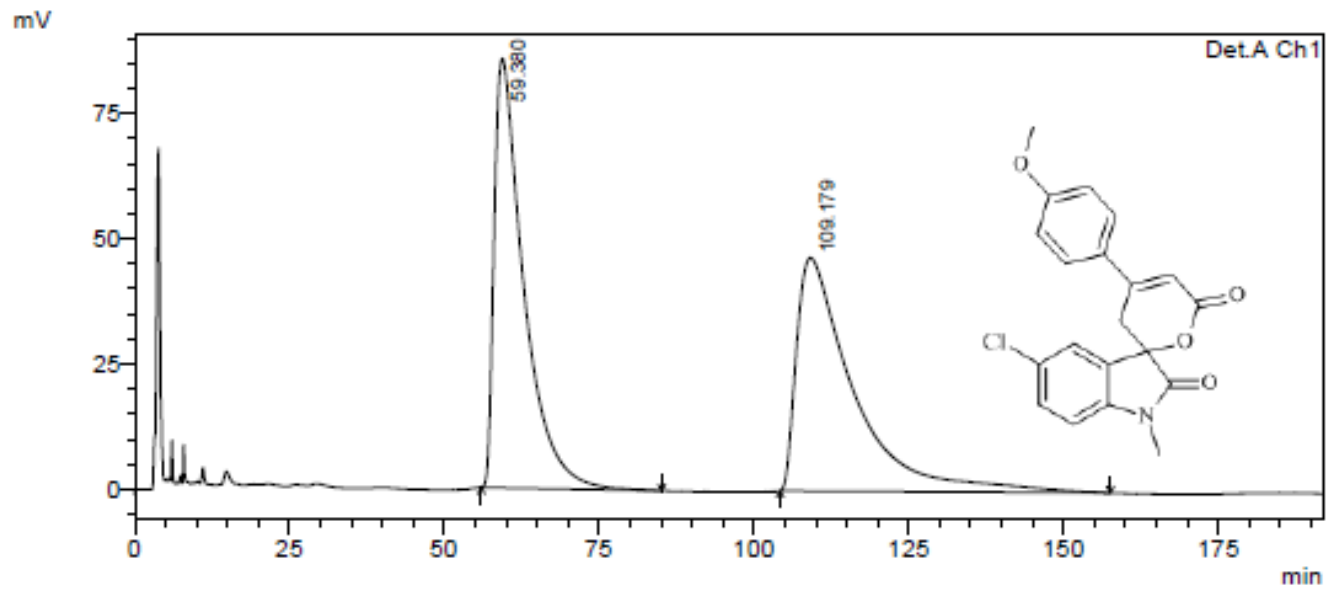

\begin{tabular}{|r|r|r|r|r|r|}
\hline \multicolumn{1}{|c|}{ Pealk } & Ret. Time & \multicolumn{1}{c|}{ Area } & \multicolumn{1}{c|}{ Height } & \multicolumn{1}{c|}{ Area \% } & \multicolumn{1}{c|}{ Height \% } \\
\hline 1 & 59.380 & 28444897 & 85727 & 48.861 & 64.780 \\
\hline 2 & 109.179 & 29770884 & 46609 & 51.139 & 35.220 \\
\hline Tota & & 58215781 & 132336 & 100.000 & 100.000 \\
\hline
\end{tabular}

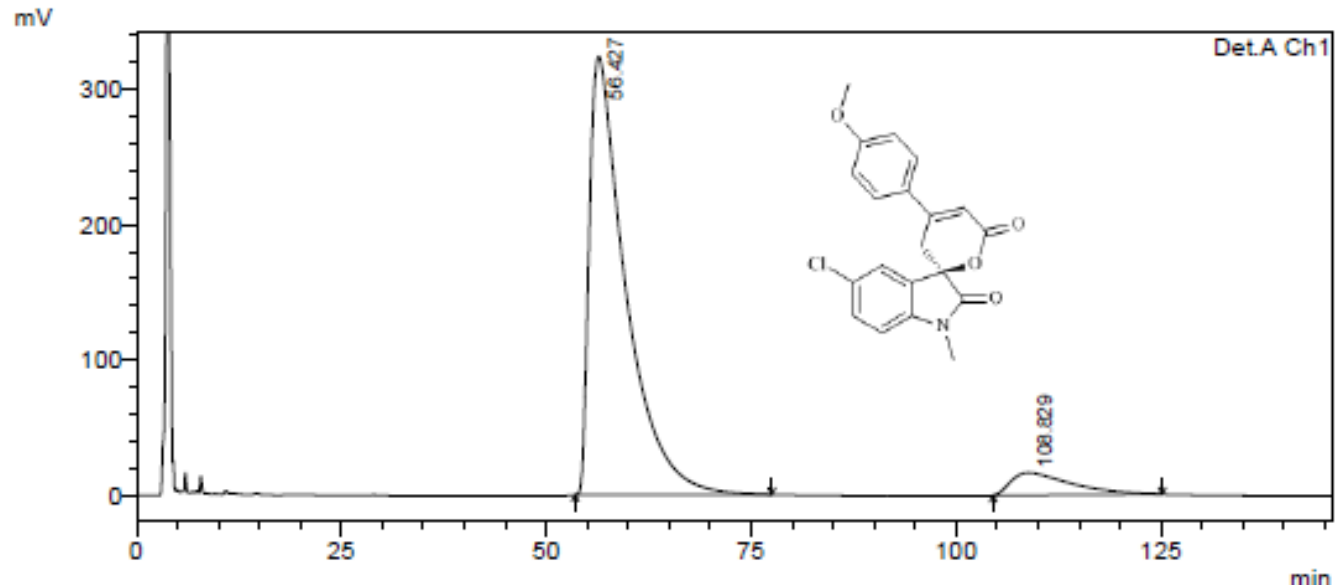

\begin{tabular}{|r|r|r|r|r|r|}
\hline \multicolumn{1}{|c|}{ Pealk $\#$} & \multicolumn{1}{|c|}{ Ret. Time } & \multicolumn{1}{c|}{ Area } & \multicolumn{1}{c|}{ Height } & \multicolumn{1}{c|}{ Area \% } & \multicolumn{1}{c|}{ Height \% } \\
\hline 1 & 56.427 & 99825255 & 323839 & 92.222 & 95.135 \\
\hline 2 & 108.829 & 8418674 & 16559 & 7.778 & 4.865 \\
\hline Total & & 108243929 & 340398 & 100.000 & 100.000 \\
\hline
\end{tabular}


(R)-4-chloro-4'-(4-methoxyphenyl)-1-methylspiro[indoline-3,2'-pyran]-2,6'(3'H)dione $(3 t)$

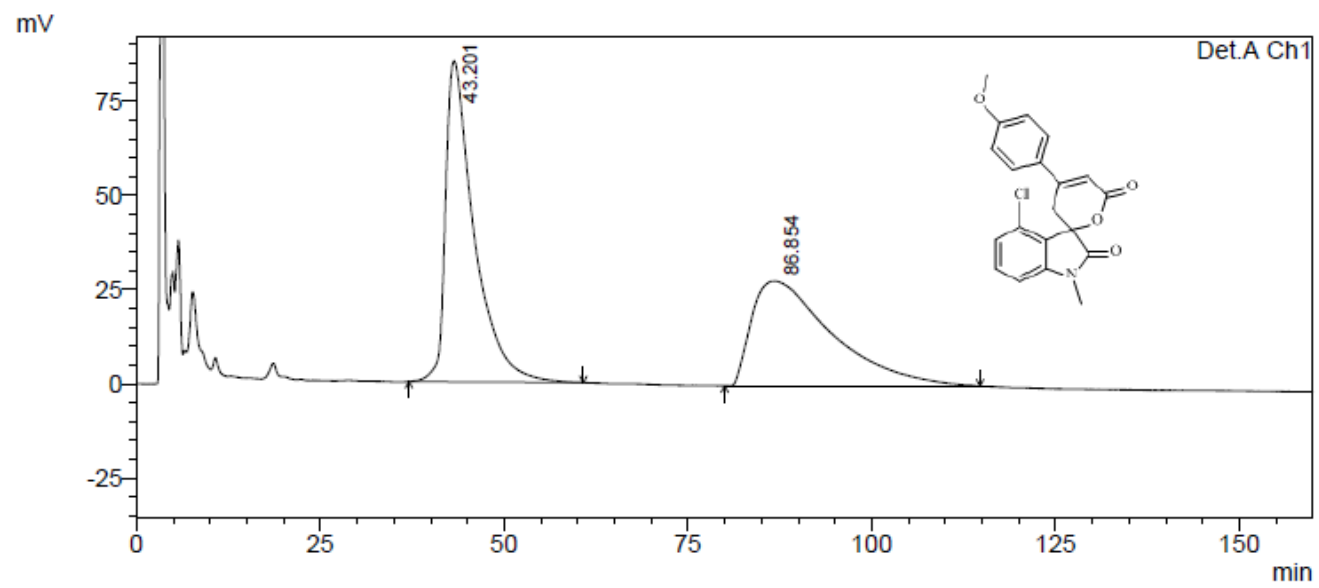

\begin{tabular}{|r|r|r|r|r|r|}
\hline \multicolumn{1}{|c|}{ Peak\# } & \multicolumn{1}{|c|}{ Ret. Time } & \multicolumn{1}{c|}{ Area } & \multicolumn{1}{c|}{ Height } & Area \% & \multicolumn{1}{c|}{ Height \% } \\
\hline 1 & 43.201 & 23052071 & 85079 & 52.175 & 75.360 \\
\hline 2 & 86.854 & 21130358 & 27818 & 47.825 & 24.640 \\
\hline Total & & 44182429 & 112896 & 100.000 & 100.000 \\
\hline
\end{tabular}

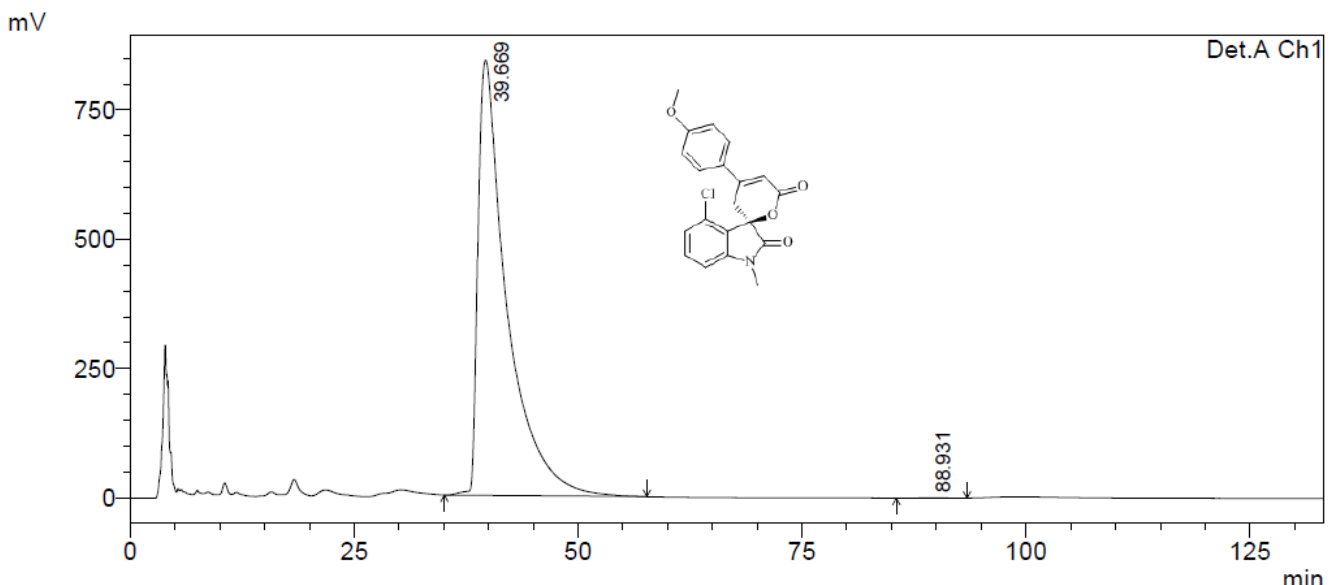

\begin{tabular}{|r|r|r|r|r|r|}
\hline \multicolumn{1}{|c|}{ Peak\# } & Ret. Time & \multicolumn{1}{c|}{ Area } & \multicolumn{1}{c|}{ Height } & \multicolumn{1}{c|}{ Area \% } & \multicolumn{1}{c|}{ Height \% } \\
\hline 1 & 39.669 & 188133851 & 841258 & 99.984 & 99.980 \\
\hline 2 & 88.931 & 29173 & 167 & 0.016 & 0.020 \\
\hline Total & & 188163024 & 841426 & 100.000 & 100.000 \\
\hline
\end{tabular}


(R)-5-bromo-4'-(4-methoxyphenyl)-1-methylspiro[indoline-3,2'-pyran]-2,6' (3'H)dione $(3 \mathbf{u})$

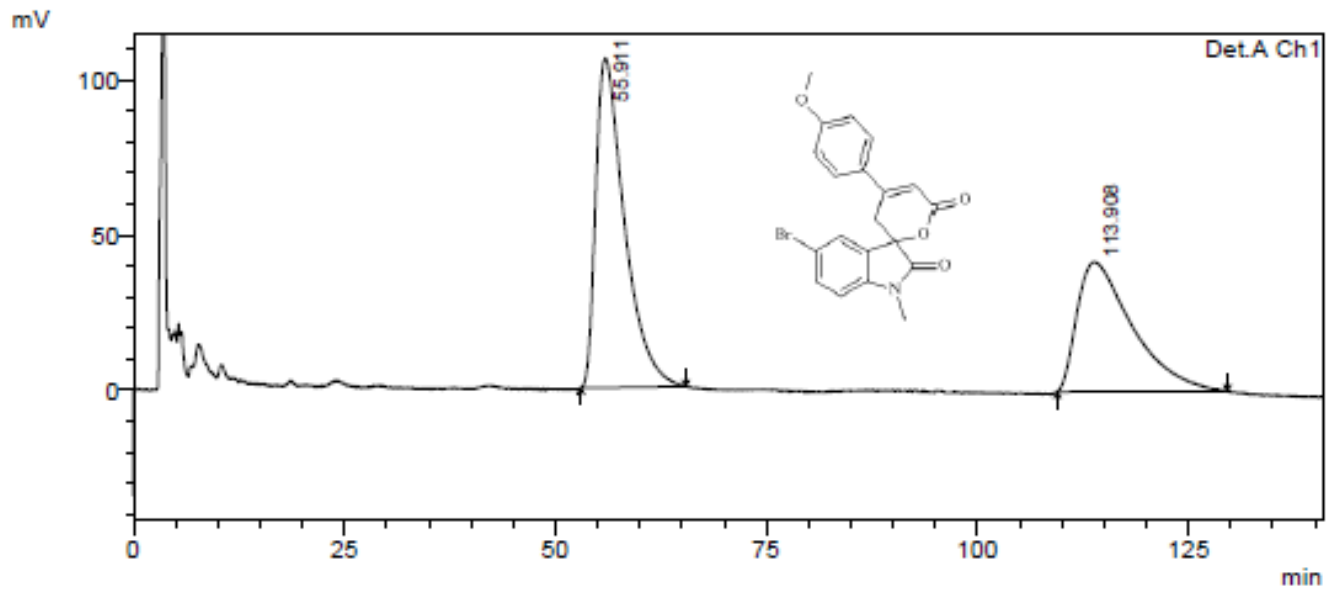

\begin{tabular}{|r|r|r|r|r|r|}
\hline \multicolumn{1}{|c|}{ PealF } & Ret. Time & \multicolumn{1}{|c|}{ Area } & \multicolumn{1}{c|}{ Height } & Area \% & \multicolumn{1}{c|}{ Height \% } \\
\hline 1 & 55.911 & 25221465 & 106234 & 56.981 & 71.743 \\
\hline 2 & 113.908 & 19041436 & 41842 & 43.019 & 28.257 \\
\hline Total & & 44262901 & 148076 & 100.000 & 100.000 \\
\hline
\end{tabular}

$\mathrm{mV}$

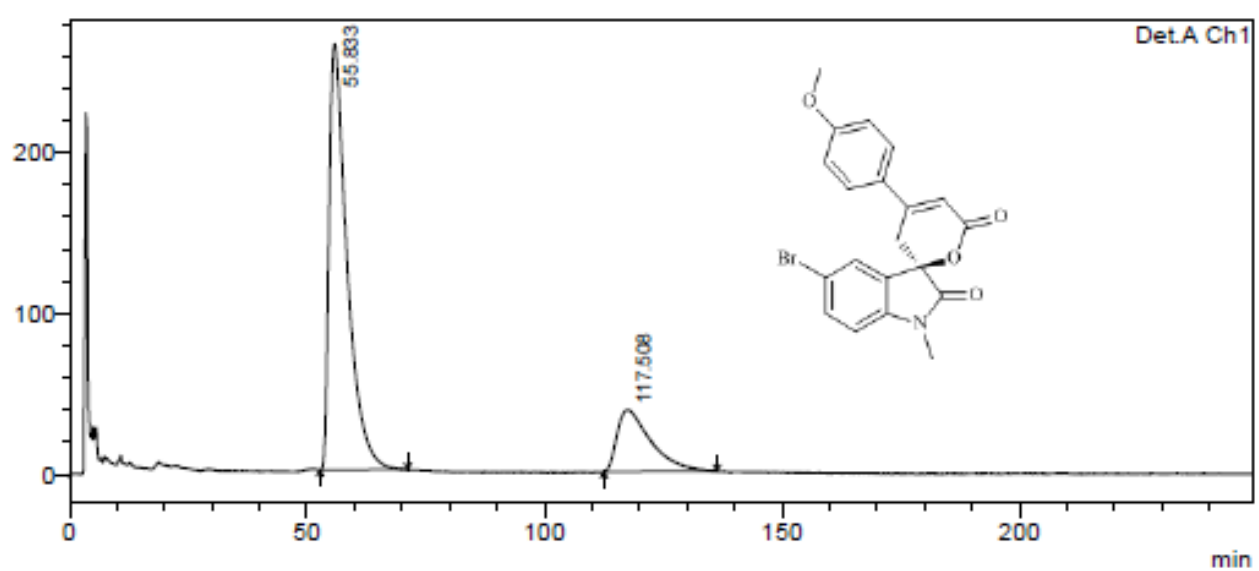

\begin{tabular}{|r|r|r|r|r|r|}
\hline \multicolumn{1}{|c|}{ Peals $=$} & \multicolumn{1}{|c|}{ Ret. Time } & \multicolumn{1}{c|}{ Area } & \multicolumn{1}{c|}{ Height } & \multicolumn{1}{c|}{ Area \% } & \multicolumn{1}{c|}{ Height \% } \\
\hline 1 & 55.833 & 69733264 & 264452 & 78.760 & 87.354 \\
\hline 2 & 117.508 & 18805798 & 38283 & 21.240 & 12.646 \\
\hline Tota & & 88539062 & 302736 & 100.000 & 100.000 \\
\hline
\end{tabular}


(R)-5-methoxy-4'-(4-methoxyphenyl)-1-methylspiro[indoline-3,2'-pyran]-2,6'(3'

H)-dione(3v)

$\mathrm{mv}$

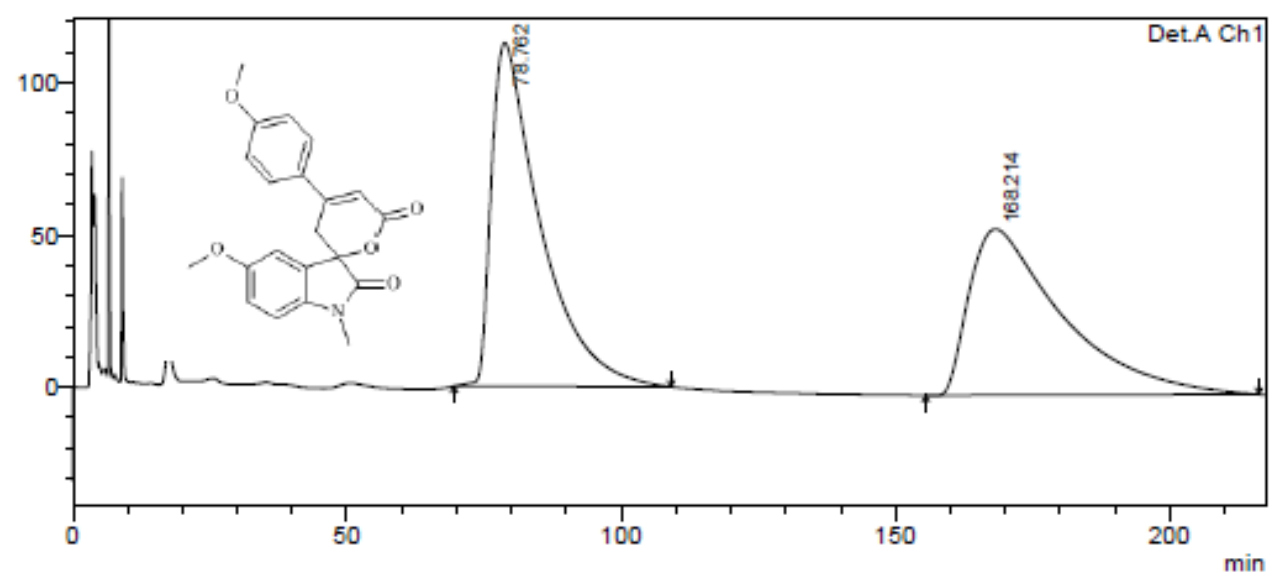

\begin{tabular}{|r|r|r|r|r|r|}
\hline \multicolumn{1}{|c|}{ Pealk $=$} & \multicolumn{1}{|c|}{ Ret. Time } & \multicolumn{1}{c|}{ Area } & Height & \multicolumn{1}{c|}{ Area \% } & \multicolumn{1}{c|}{ Height \% } \\
\hline 1 & 78.762 & 70157796 & 112989 & 52.174 & 67.402 \\
\hline 2 & 168.214 & 64311088 & 54646 & 47.826 & 32.598 \\
\hline Total & & 134468883 & 167636 & 100.000 & 100.000 \\
\hline
\end{tabular}

$\mathrm{mV}$

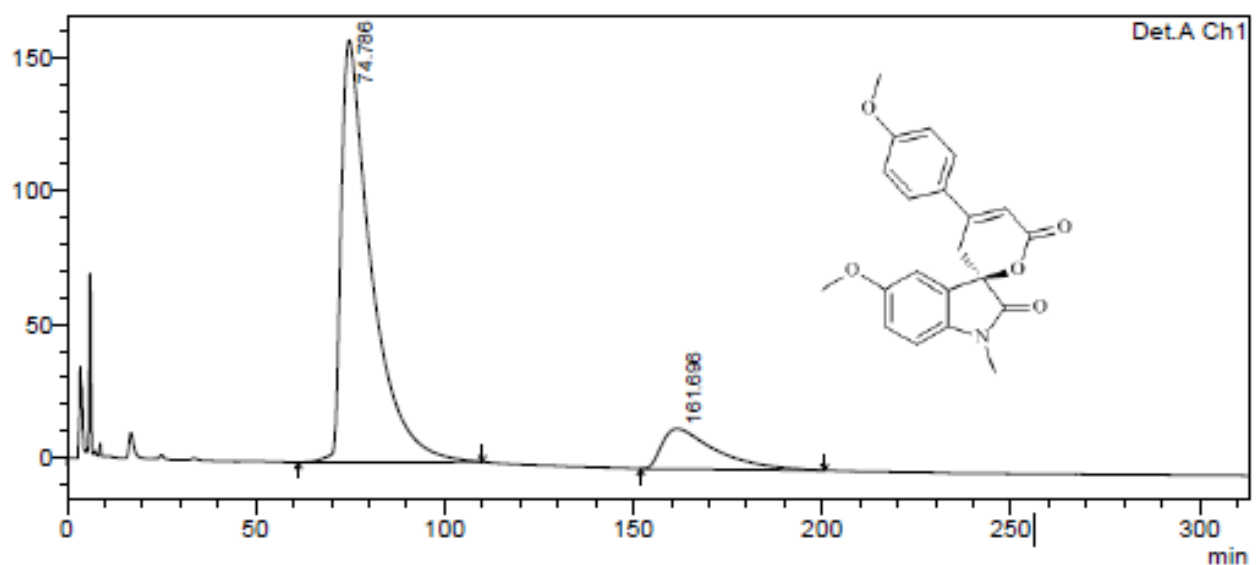

\begin{tabular}{|r|r|r|r|r|r|}
\hline \multicolumn{1}{|c|}{ Peals= } & \multicolumn{1}{|c|}{ Ret. Time } & \multicolumn{1}{c|}{ Area } & \multicolumn{1}{c|}{ Height } & \multicolumn{1}{c|}{ Area \% } & \multicolumn{1}{c|}{ Height \% } \\
\hline 1 & 74.786 & 87652898 & 158087 & 85.502 & 91.263 \\
\hline 2 & 161.696 & 14862865 & 15134 & 14.498 & 8.737 \\
\hline Total & & 102515762 & 173220 & 100.000 & 100.000 \\
\hline
\end{tabular}


(R)-1,4'-diphenylspiro[indoline-3,2'-pyran]-2,6'(3'H)-dione(3w).

$\mathrm{mV}$

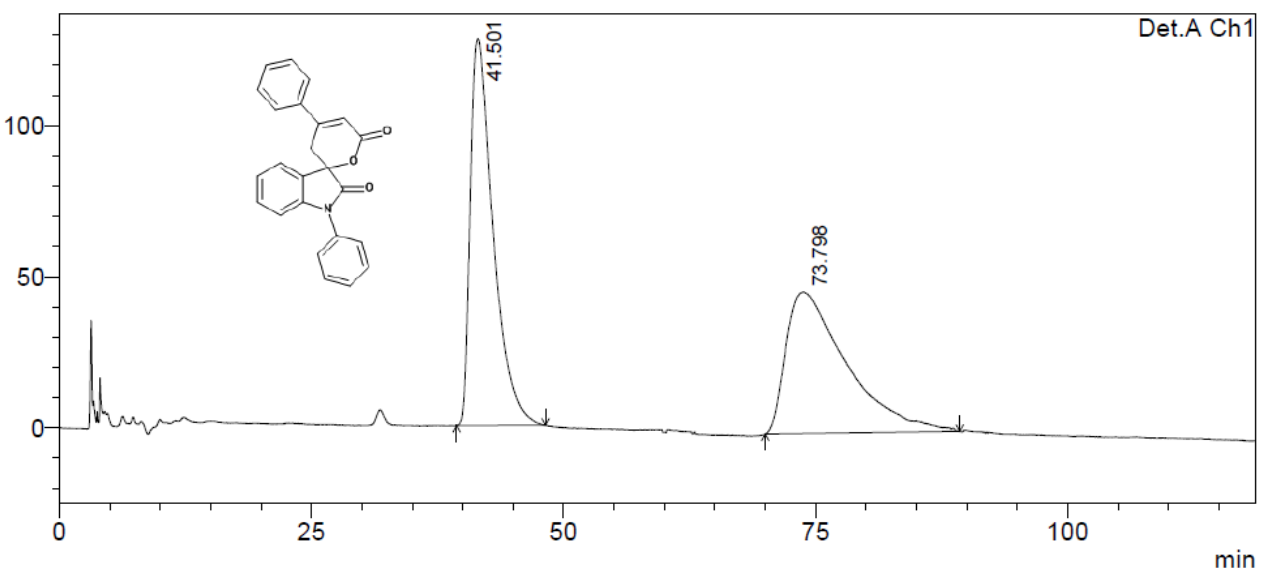

\begin{tabular}{|r|r|r|r|r|r|}
\hline \multicolumn{1}{|c|}{ Peak\# } & Ret. Time & \multicolumn{1}{|c|}{ Area } & Height & Area \% & \multicolumn{1}{c|}{ Height \% } \\
\hline 1 & 41.501 & 20235003 & 128043 & 50.763 & 73.160 \\
\hline 2 & 73.798 & 19626963 & 46976 & 49.237 & 26.840 \\
\hline Total & & 39861967 & 175019 & 100.000 & 100.000 \\
\hline
\end{tabular}

$\mathrm{mV}$

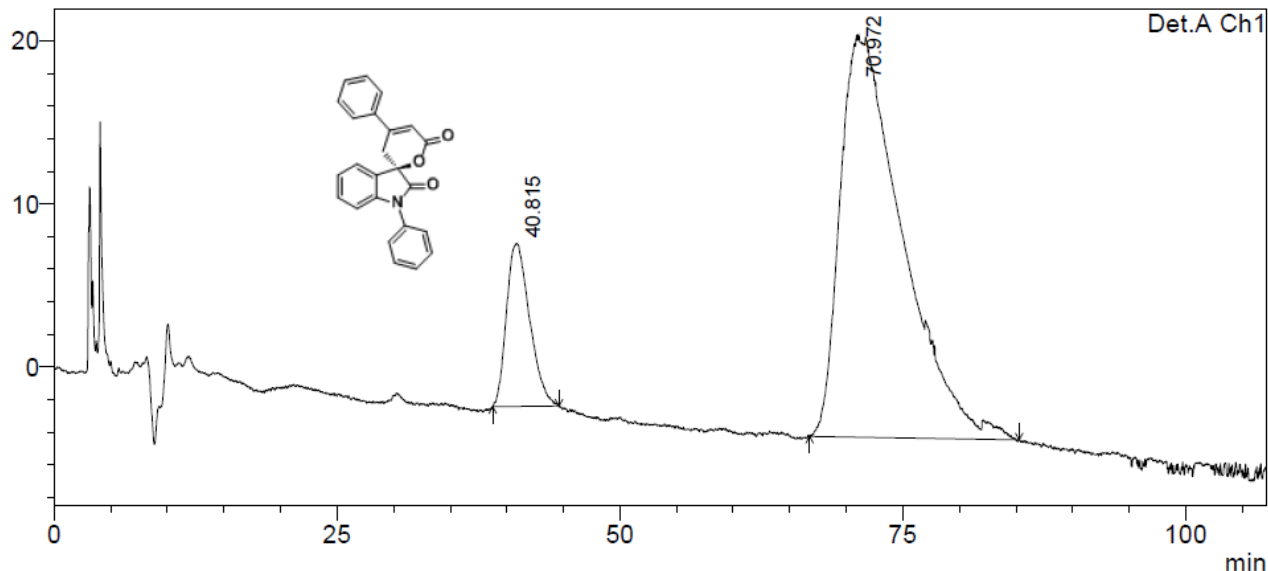

\begin{tabular}{|r|r|r|r|r|r|}
\hline \multicolumn{1}{|c|}{ Peak\# } & Ret. Time & \multicolumn{1}{|c|}{ Area } & \multicolumn{1}{c|}{ Height } & Area \% & \multicolumn{1}{c|}{ Height $\%$} \\
\hline 1 & 40.815 & 1426340 & 10005 & 13.126 & 28.819 \\
\hline 2 & 70.972 & 9440574 & 24712 & 86.874 & 71.181 \\
\hline Total & & 10866914 & 34718 & 100.000 & 100.000 \\
\hline
\end{tabular}

